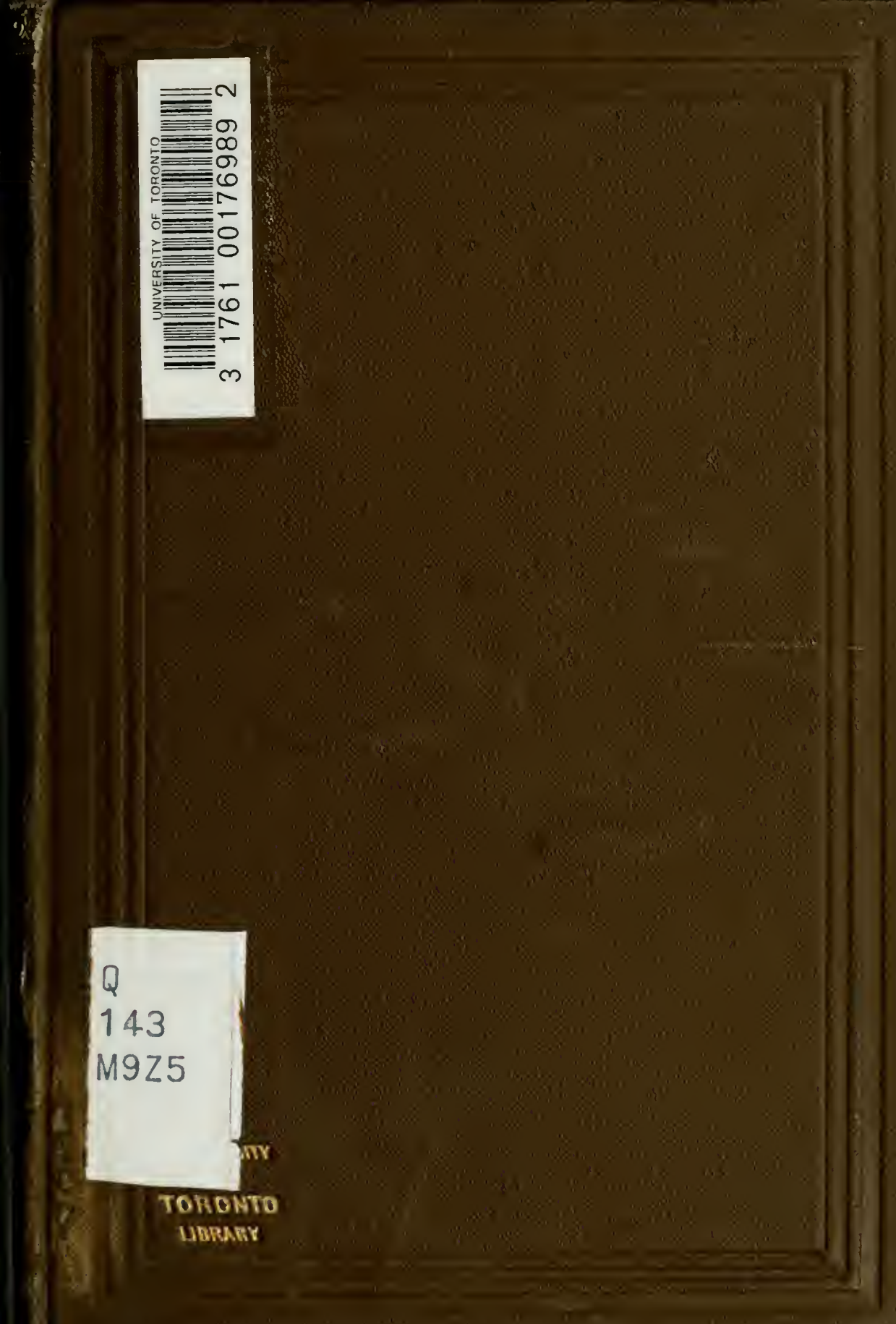







\title{
Reninmuntan,
}

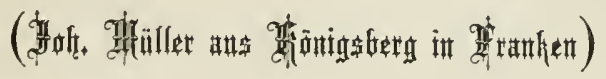

ein

\section{geiftiger Borläufer dez (5olumbus.}

\author{
Bon
}

Pllerantiox 3iegler.
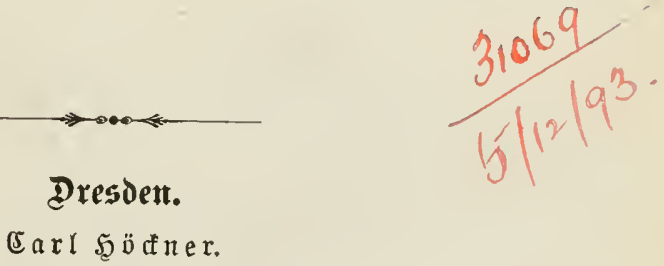

1874. 


$$
\begin{aligned}
& Q \\
& 143 \\
& M 925
\end{aligned}
$$




\section{5errn \\ Dr. $\mathfrak{d} \mathfrak{x} \mathfrak{e} \mathfrak{\mathfrak { i }} \mathfrak{i} \mathfrak{g}$,}

Director Der Steuermanmighule in $B$ remen

ein 3rifhen

feiner fjodhadjtung.

จุนนตุโa,

1. März 1874 .

Qlexanior 3iegler. 



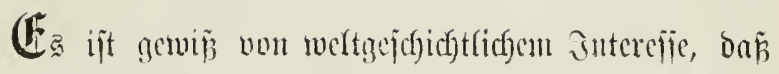

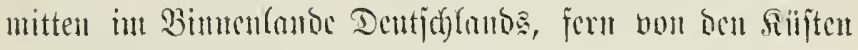

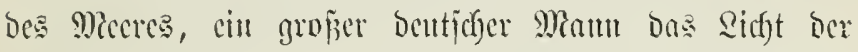
Welt erblifft bat, ber ars Refurmator ber Eterufunde

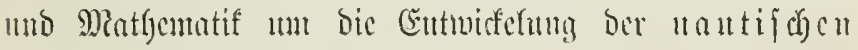
2fitronomie unb ber bamit in engiter Berbintoung ftebuben oceanijgen Saffffagrt bie gröpten Ber= bienjte gefabt fat. Dicjer grop̌e beutjche Mann naunte fich Regionoutanu. Durdy feine fpätere lleberjiebelung

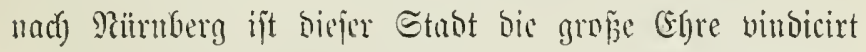

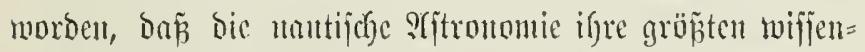

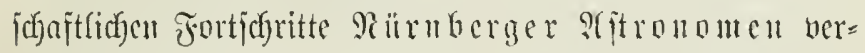

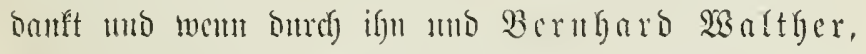
eimen Echüler bes Regiomnutants, bas Etmbinm der afjtronomie mo Mathematif zucrit an Piïruberg gefeffelt

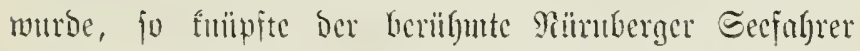
Martin Befaim - atd) cin Eefüler bes Regionton=

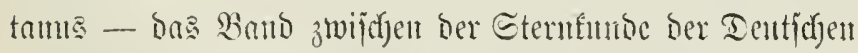
uno ber Miautif ber Evanicr mo Rortugiefm.

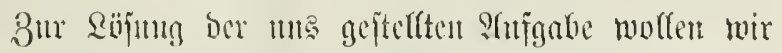

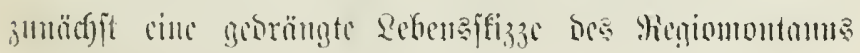




\section{$-\quad 2 \ldots$}

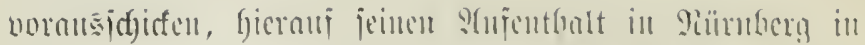

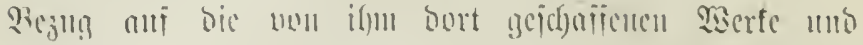

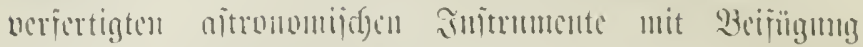

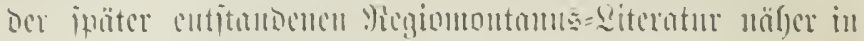

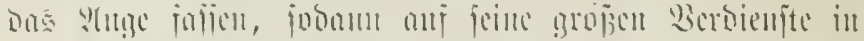

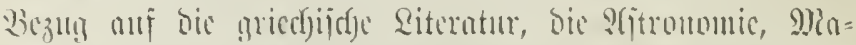

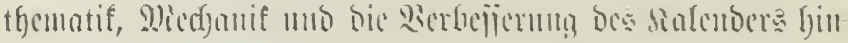

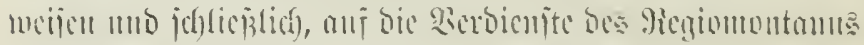

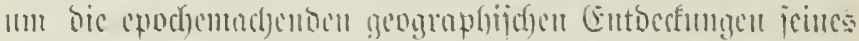

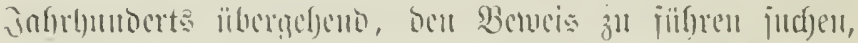

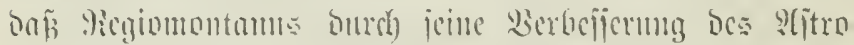

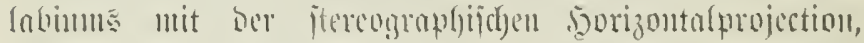

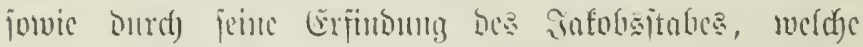

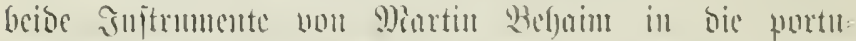

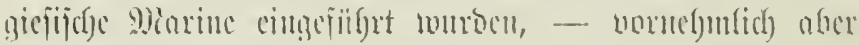

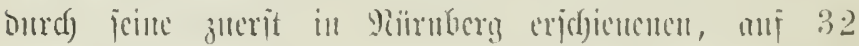

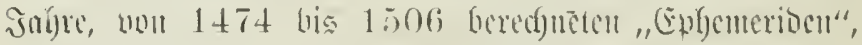

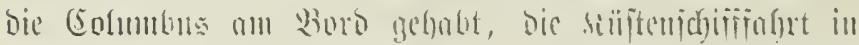

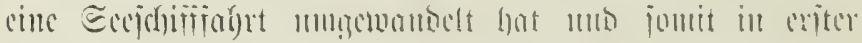

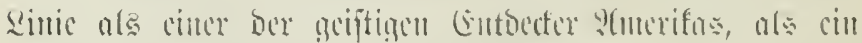

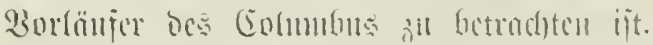

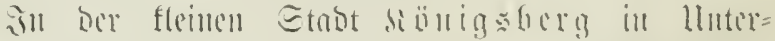

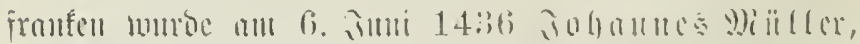

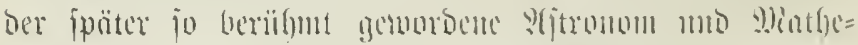

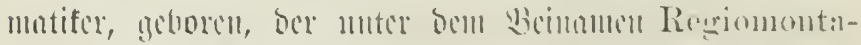

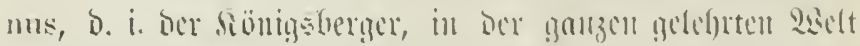

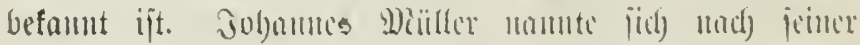


Bateritud Regiomontanus, unch) do monte Regio, Jo:mnes de Regiomonte, biftir nuch IIans de Kungsherg, Königsberg, Kunisperger, Germanns nocr Francus, mitunter, wic b. Mimre angibt, and Miller

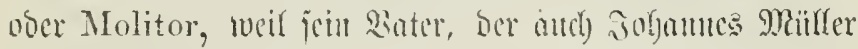

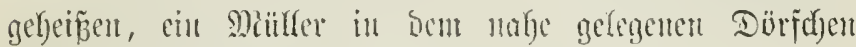

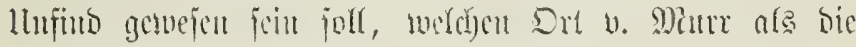

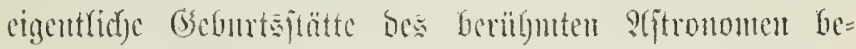

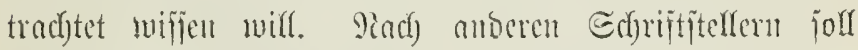

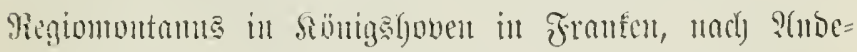

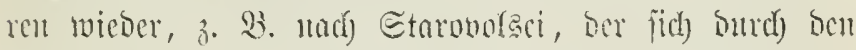

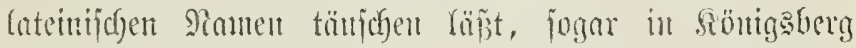

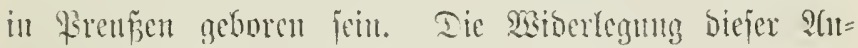

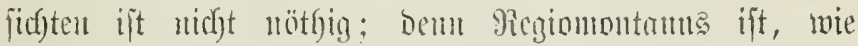

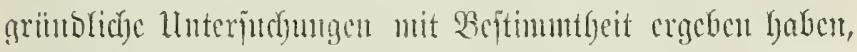

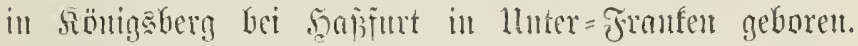

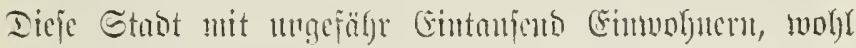
bie flemite unter Den zuhfreschent Etribten gleid)en

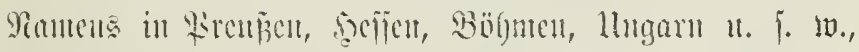

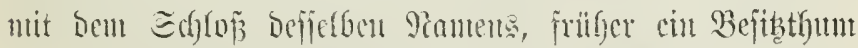

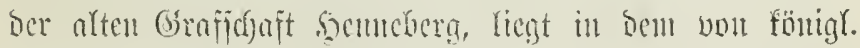

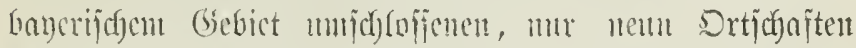

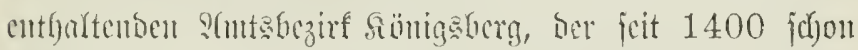

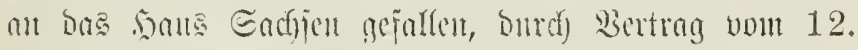

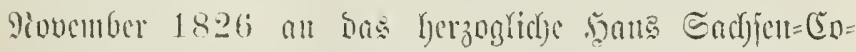

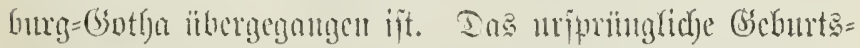

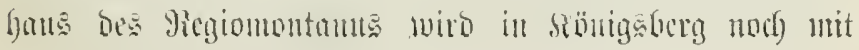




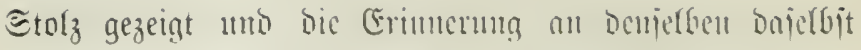
ถurd) cine Regiommunm 1871 gegrïnbetes mïrbiges Icufmal*) mo burd) cincu

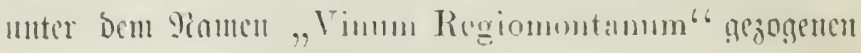
recin mad)yeruje

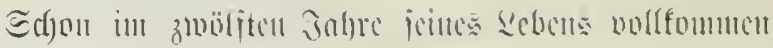

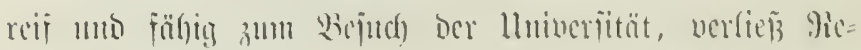

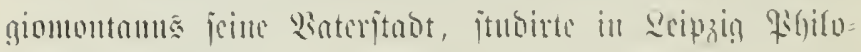

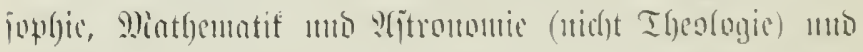

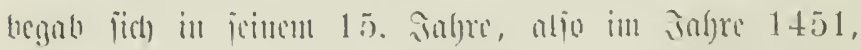

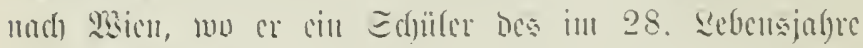

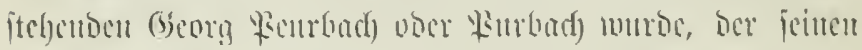

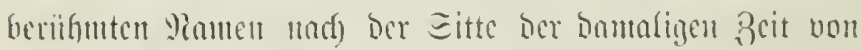

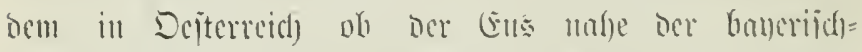

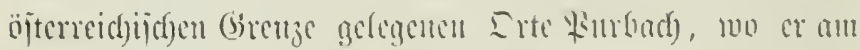

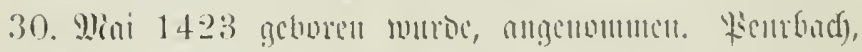

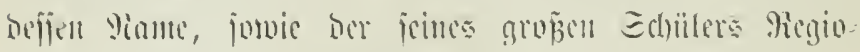

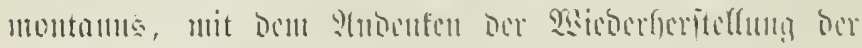

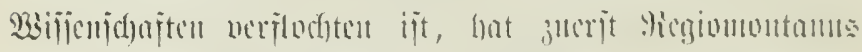

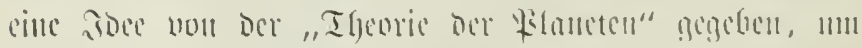

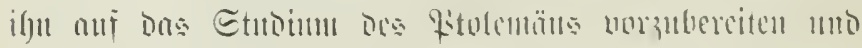

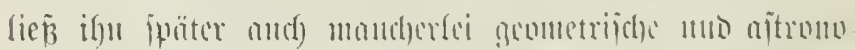

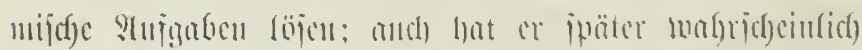

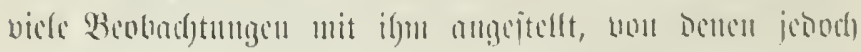

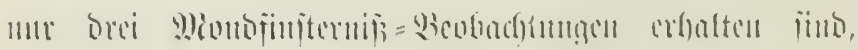

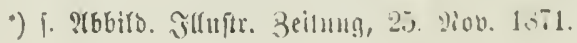


(virgl. Willebr. Suellius: coeli et sidermm in eo errantium observat. Hassiacae. Luged. 1618 p. 12 et 14).

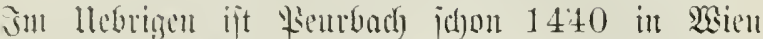

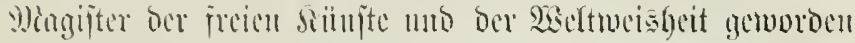

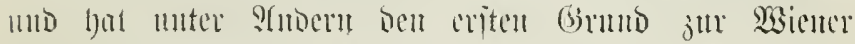

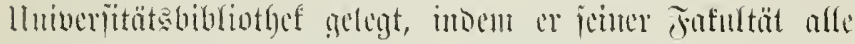

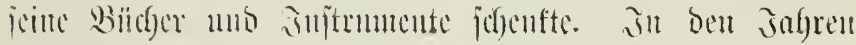

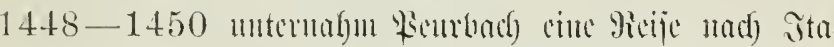

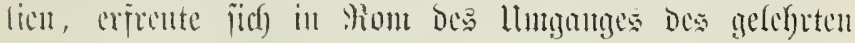

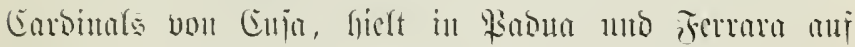

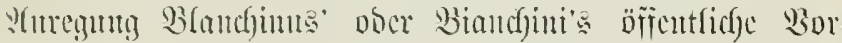

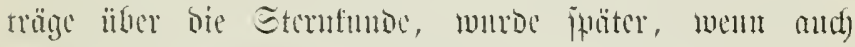

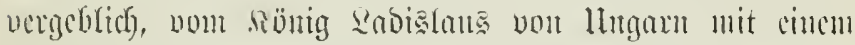

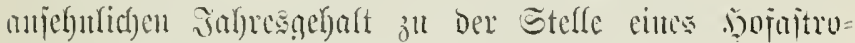
nomen berufen

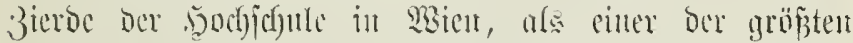

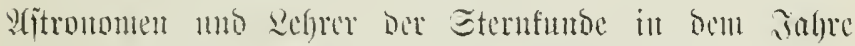

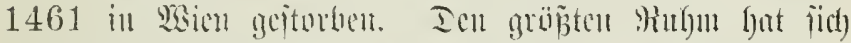

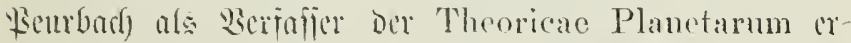

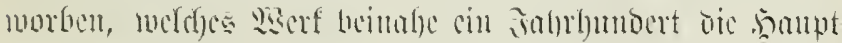

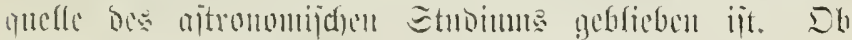

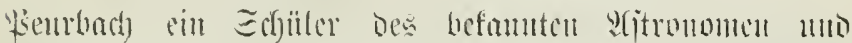

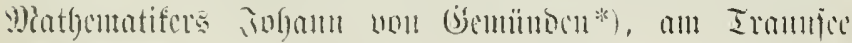

* Johannes de Gmunden ober de Gemunde (Soljann ober

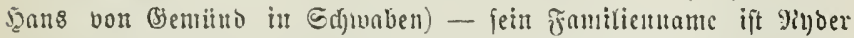

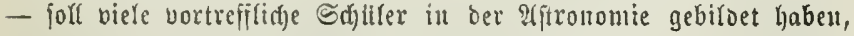

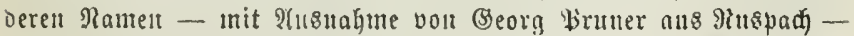

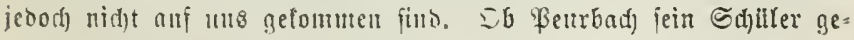




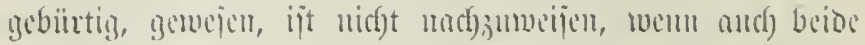

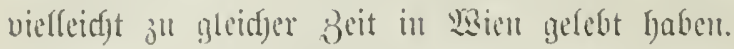

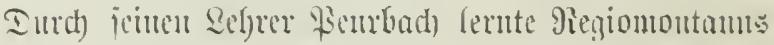
Den beriffnten 1395) ju Irapezunt geborenen (Bried)en,

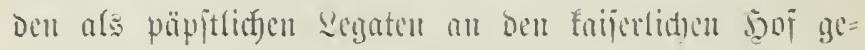

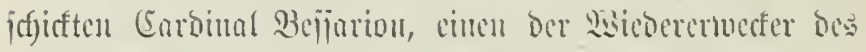

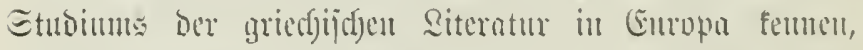

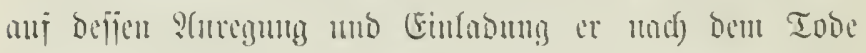
Peurbad) nad) itulien rejp. Bom ging. Injelbjt jtu= birte gregionuntaus bie apriedjijde Epradje mit dem gröpten Cijer, jummelte sicle Miamnjeripte, vollendete bie

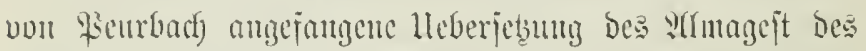

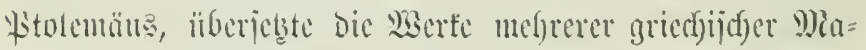
thematifer suic dic des Afpollonios Eonicos, Eerenos (E)=

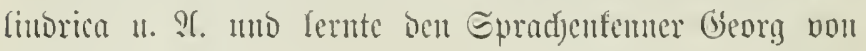
Trcpezunt femten, der gerabe bamale wit eiter latei=

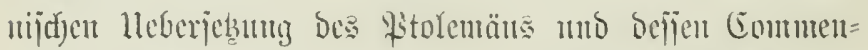

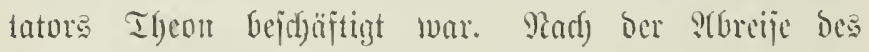
Earbinals Befjarion begab jidf) Bicginumbutames (vou Toun?) nadl Ferrara. Dier lernte er ben fojt $90=$

Ivejen, bilrite fehr zucifelfaft jeiu. Das (3eburtsjahr von Sohames

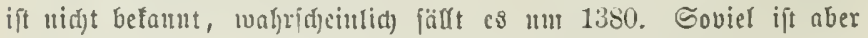

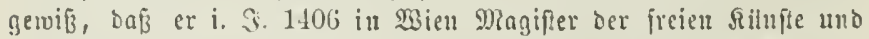
ber \$hilofoplie untoe uto feit ber 3cit 9fpronomie lehrte. Er ftarb Den 23. Febr. 1442. 2ou jeinen @driften ift teine cinjige burd) Den Drud befant geworoen, bis auf einen gebrufícu Salenter in laleinifjer Epradje (i. Etern in Der Encyflopätic von Expd uto (3) ruber. Siveite Gection nuter: Johannes de Ginunden. 


\section{$-i-$}

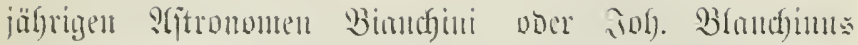

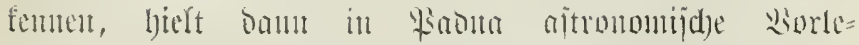

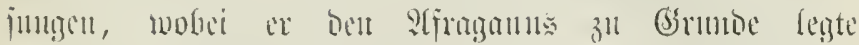

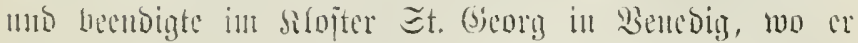

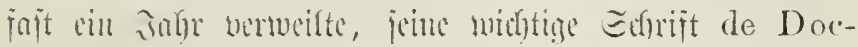
trina Triangulorum vode de triangulis i. 3. 1463. jon Folge biejes Sonutmertes ber mathematị̆chen

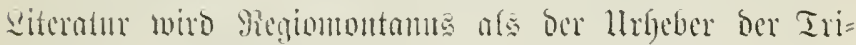

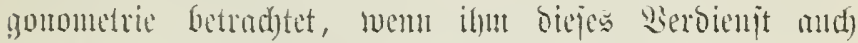
oer Itutiener Formalenui in jemem .Saggio sulla Nan-

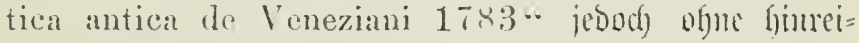

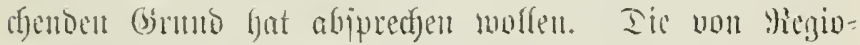

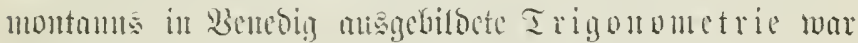

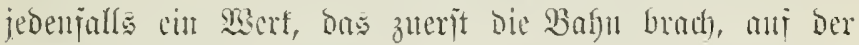

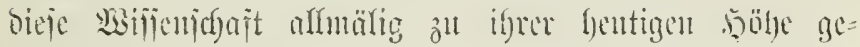

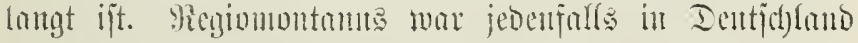
Der (Erite, ber ïd) mit (Eifor anj bas Etmoimm mo dic

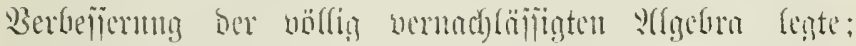

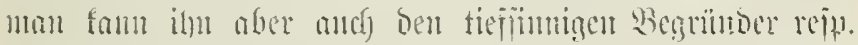
Berbolffomminer Der Irigonometric nemen, Der nantest=

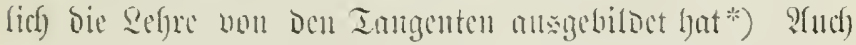

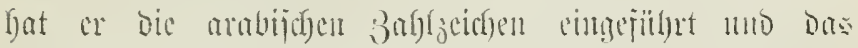

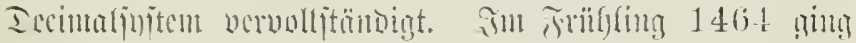

*) Regiomontanus a depuis, d'après le gnomon de Purbach fait une table de tangentes pour tous les dógres du quart de cercle. 11 lajpelle Table feconde; cependant il n'en fait guère d'autre usage que cului de calculer le sinus de la diffé- 


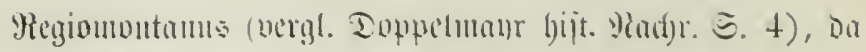

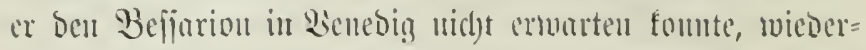

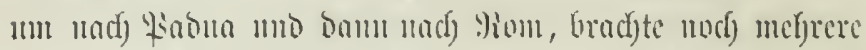

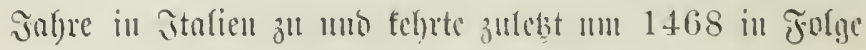

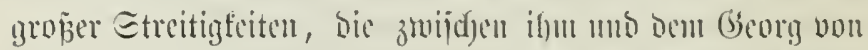

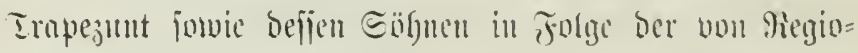

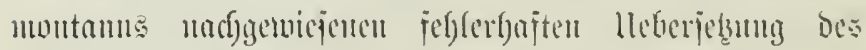

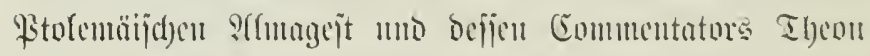

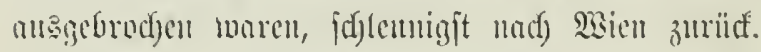

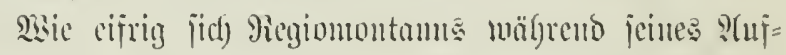
culfultes in Stalien mit ber griec(jijd)en Epradje bejd)äj=

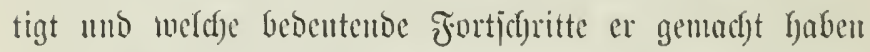

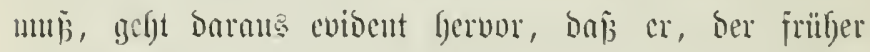
wegen jeiter llufentmin ber griedfijgen Sprad)e bie nod)

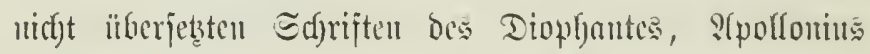

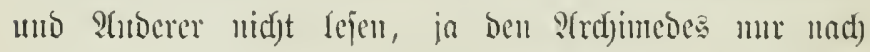

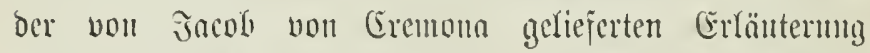

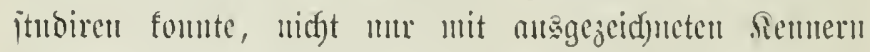
Der griecfijchen Sprache, wie Befjarion, Theoborn vou

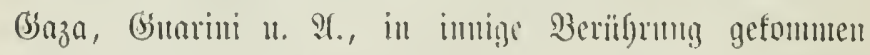

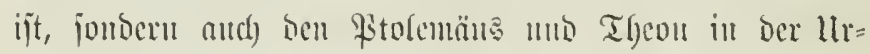
ipradje fenten gelernt, zaffreicfe griedfijd)e (Eobices ge=

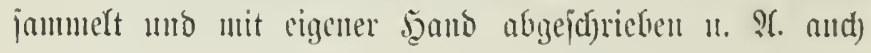

rence ascensionclle. La Table féconde de Regiomontan a été etendue par Reinhold i toutes les minutes du quart de cercle. Rheticus l'a étendue à toutes les dixaines de sécondes.

Histoire de l'astronomie du moyen age par M. Delamhre. Paris 1819 l. 294. 


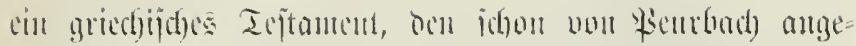
fanyetien mb jait bi

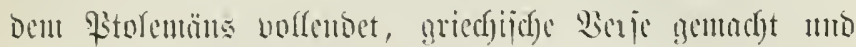

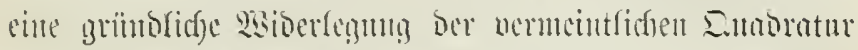
Des Cirfels, bic ber Earbinal wout Eufa in jeiner Echrijt: tractatus de quadratura cireuli gejunten granen worfte,

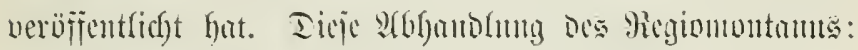
, de Quadratura circuli" i. S. 1463 max Tos=

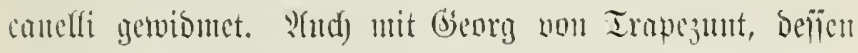

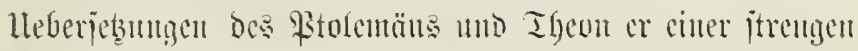

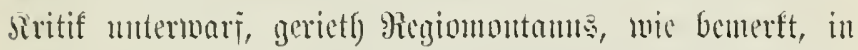
Hom in unangenefme Streitigfeiten und Bünfercien, die

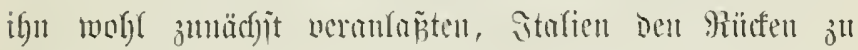
febren.

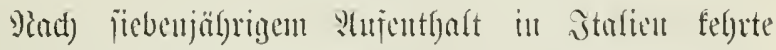

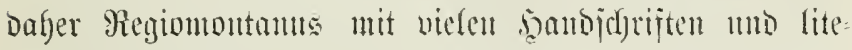

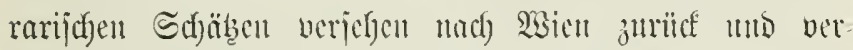

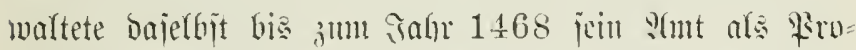

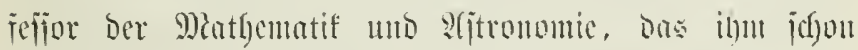

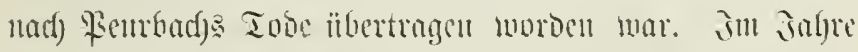

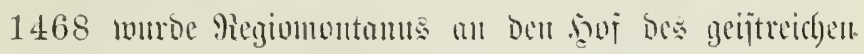

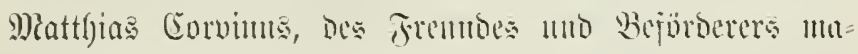

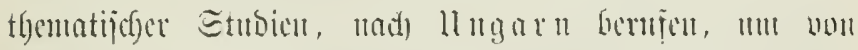

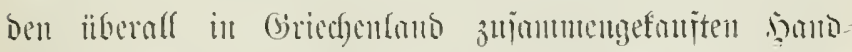

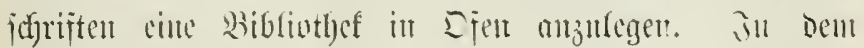

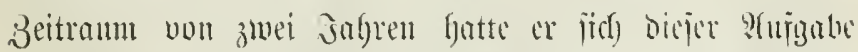

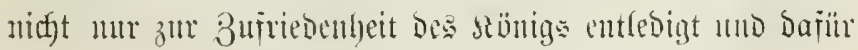




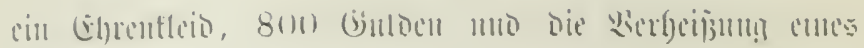

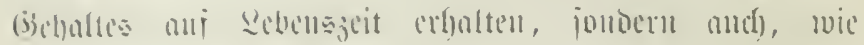
Eoppetmant angilut, bie Tabulae primi molilis fïr ocn

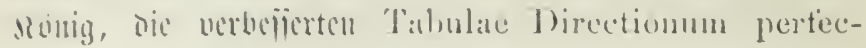

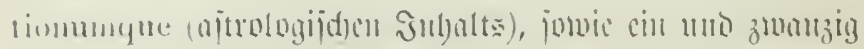

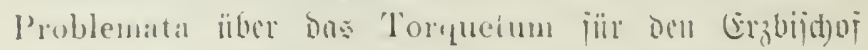

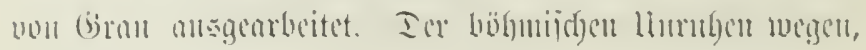

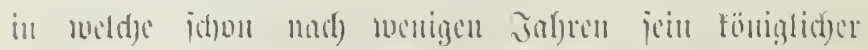

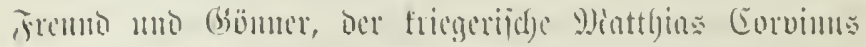

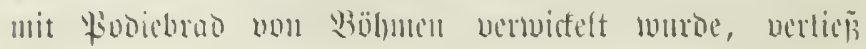

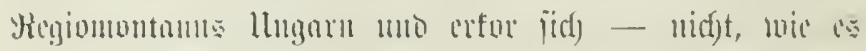

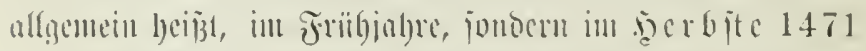

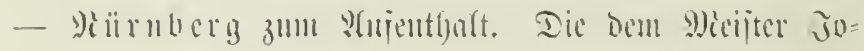

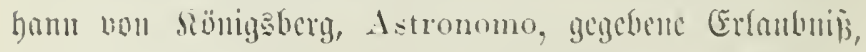

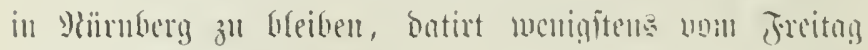
Den 29. Viovember 1471.

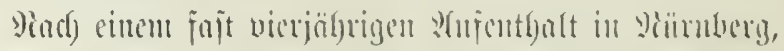

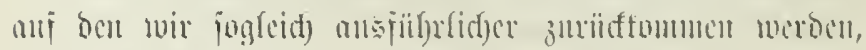

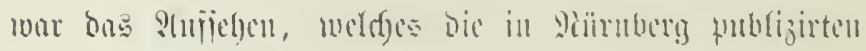
Ephemerioun gleid) mad) ifrem (end)

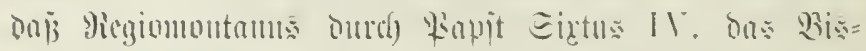

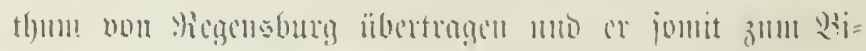

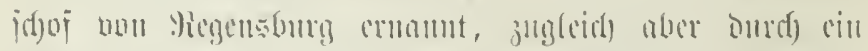

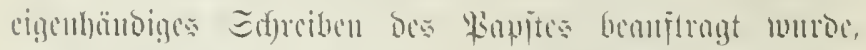

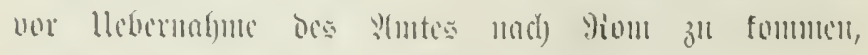

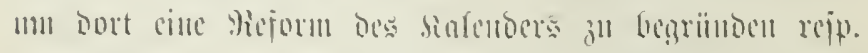

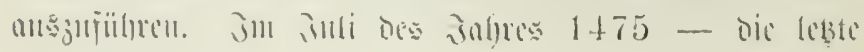




\section{$-11$}

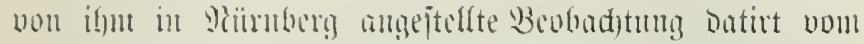

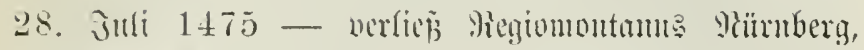

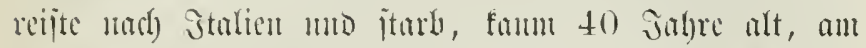

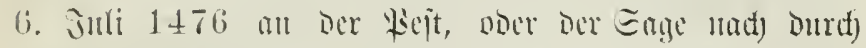

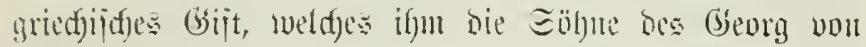

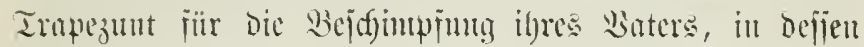

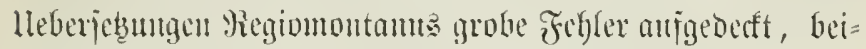

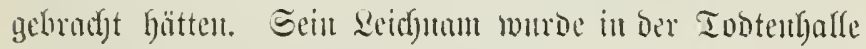

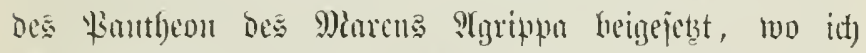

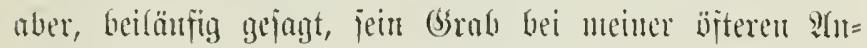

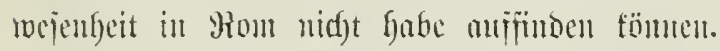

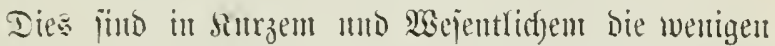

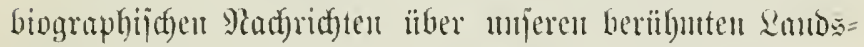

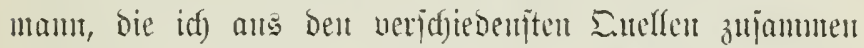

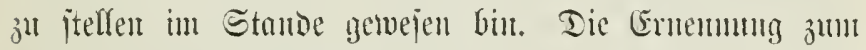

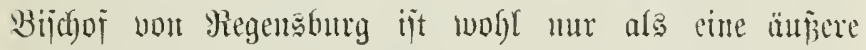

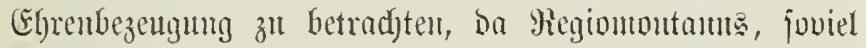
man weis, Theologie nifgt jtubirt lyat unt weent bies

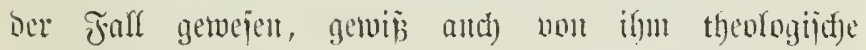

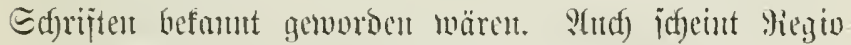

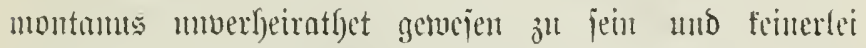

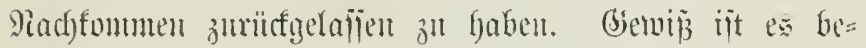

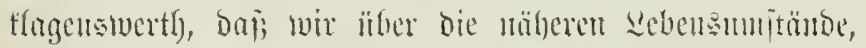

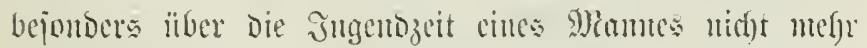

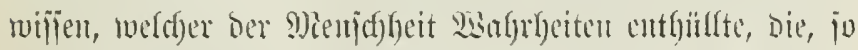

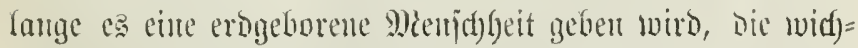

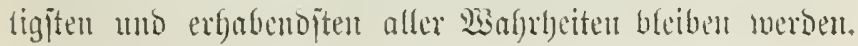




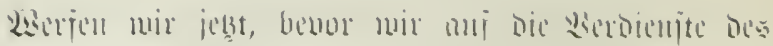

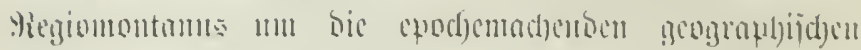

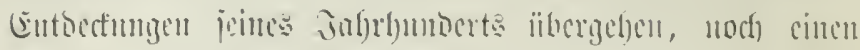

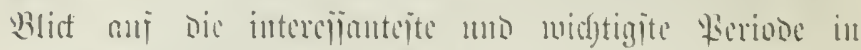

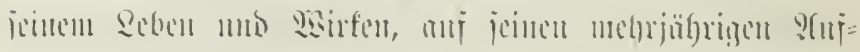

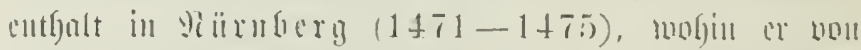

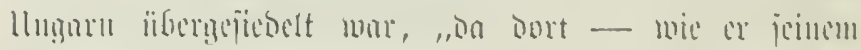

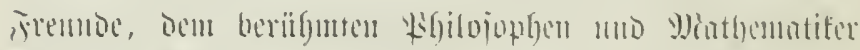

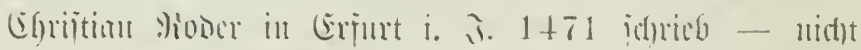

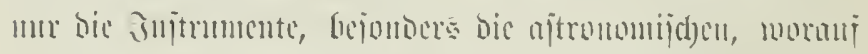

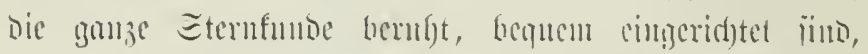

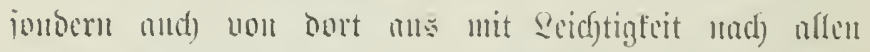

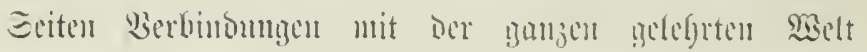
unterfalten merben fümen, benu jeuen Drt buri mun

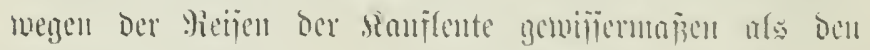

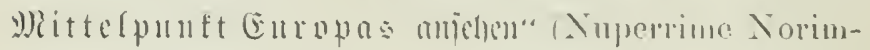
herganu mihi delegi domm perpetuam, tum propter commoditaten instrmmentormu maxime astronomi"ormun, quibus tota sideralis imititur di-ciplinal, tum propter unirersilem conversationem facilins habendam cum studionis viris uhiommene vitan degentibus.

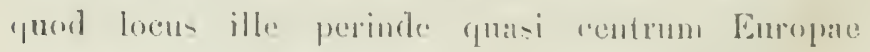

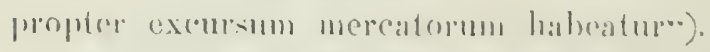

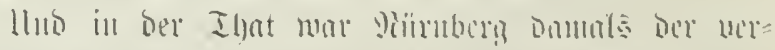

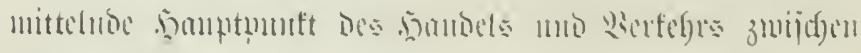

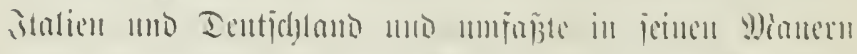

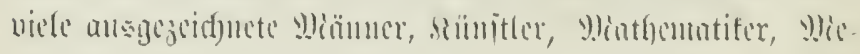




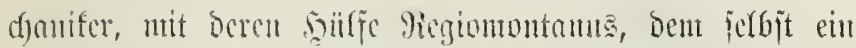

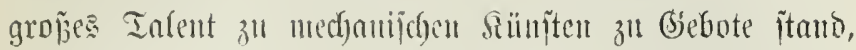

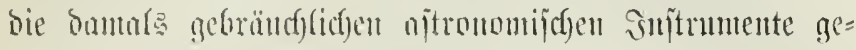

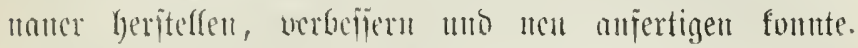

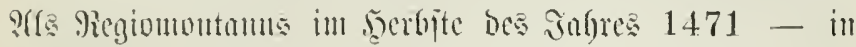

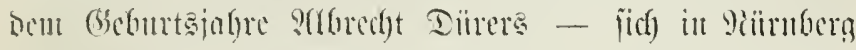

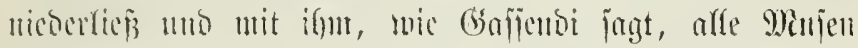

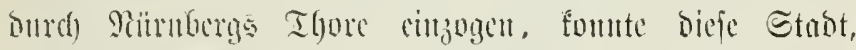
beren glüngembiti 3cif nou ber Mritte bes 15. Jafles=

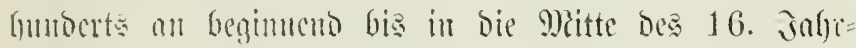

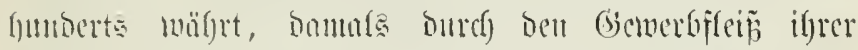

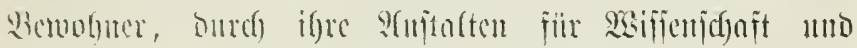

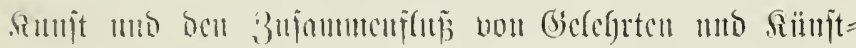

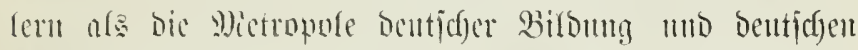

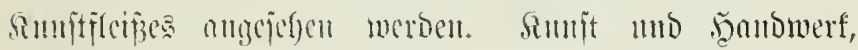

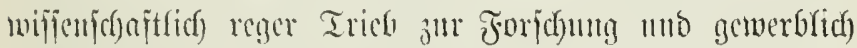

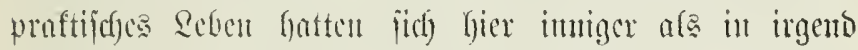

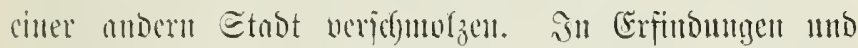

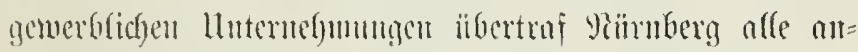
Deren Etübte.*)

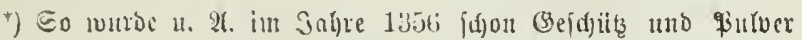
von cinem getviffen Meifter Sanger verfanft mo i. S. 1390 zu St. Sohantes $\mathfrak{T} a g$ joll Enmuenben vou ltumann Etromer, Fathsherr

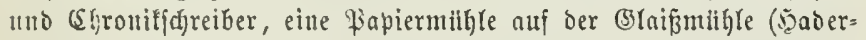
iniffle) erbaut. Sa es war bie Fapierfabrifation fdjon 1390 (f.

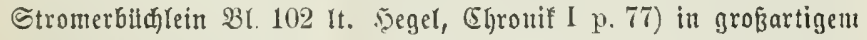
Maßftabe eingefiilfrt. Durdi bie Familie 5olbein war von giavens:

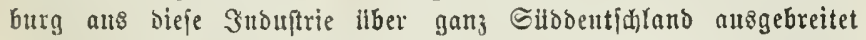
worben (vergl, Dis, Saje: Die Soburger હ. 28). Fermer erfand 


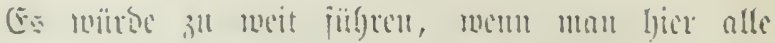

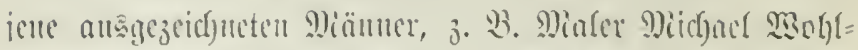

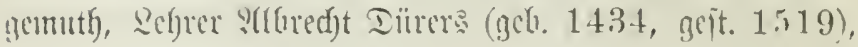

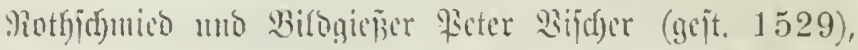

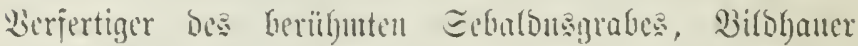

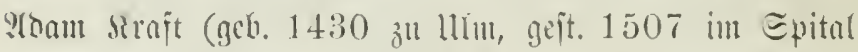
3.t1 S(f)wobad)), Derfertiger be Entrament

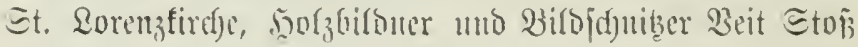

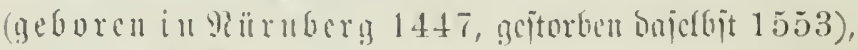

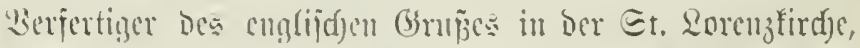

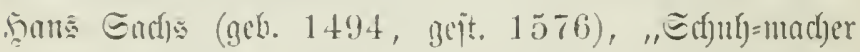

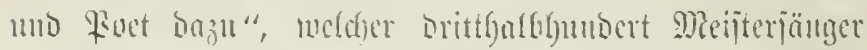

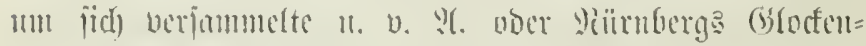

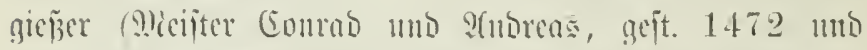

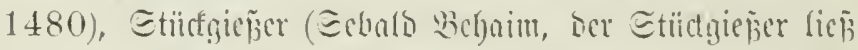

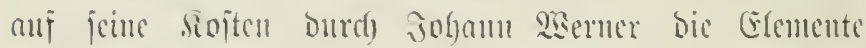

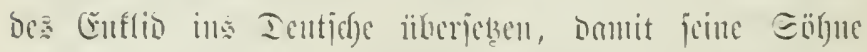

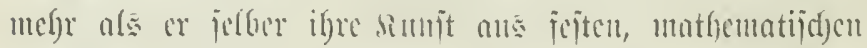

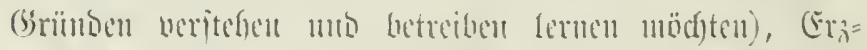

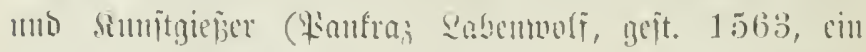

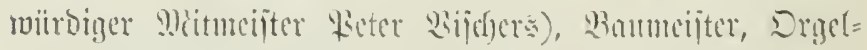

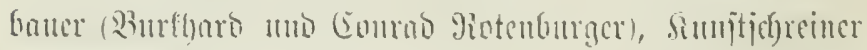

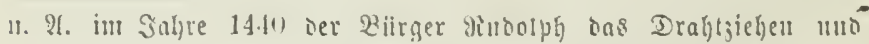

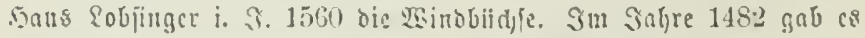

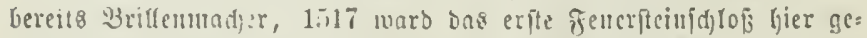

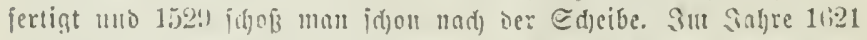

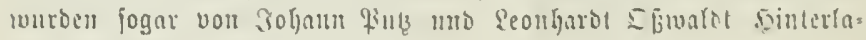
sungegewefre gefertigl. 


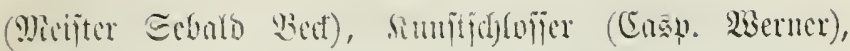

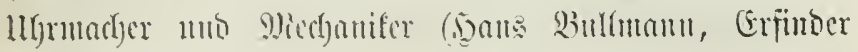

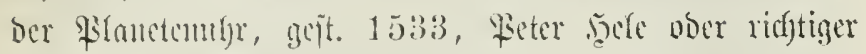

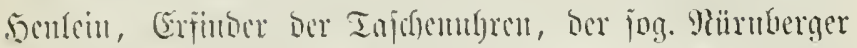

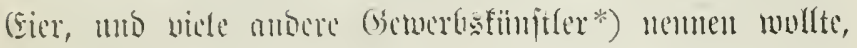

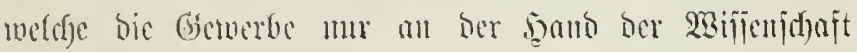

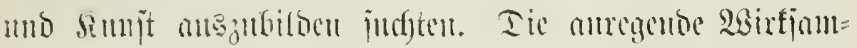

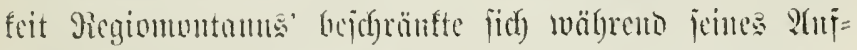

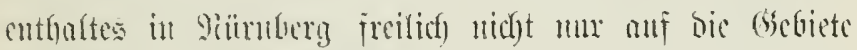

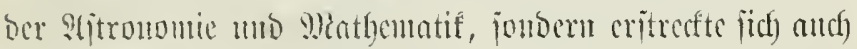

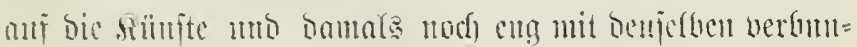

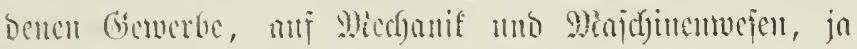

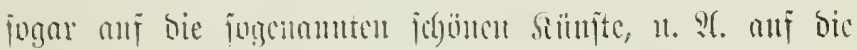

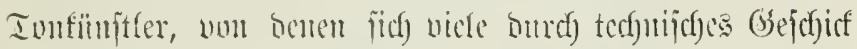

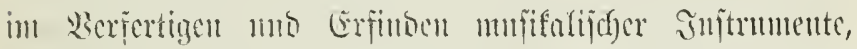

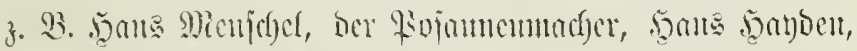

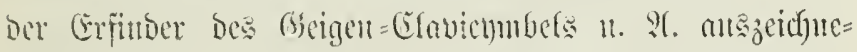
tell, wic bies Dr. (3). 5. Esfubert in jeiner geiftreidyen

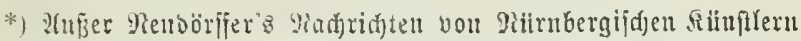
(herausgegeben von (Eampe nub it einer leiser midgt borffünoigen

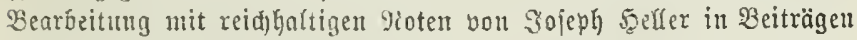

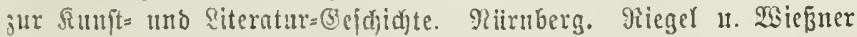

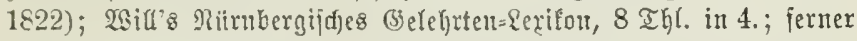

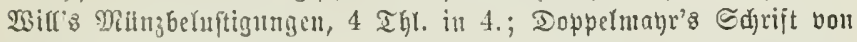

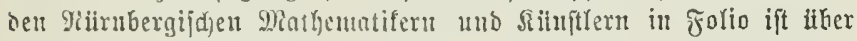
Sunft mo Siteratur in Sittruberg Das Reurejte: Sol. Baaber'\& Bei=

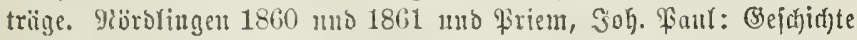
der Etabt yitruberg bon bem erfen minublidjen giadjweis igres

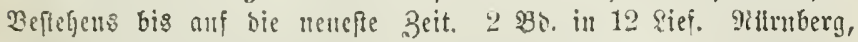
3eiiers Budh. 1873. 


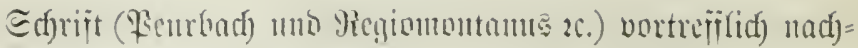
gewieju. \$on Regionnontanus, befien rajthjer reidjke=

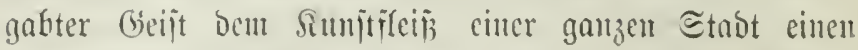

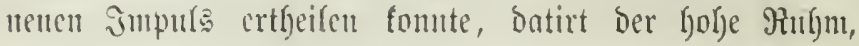

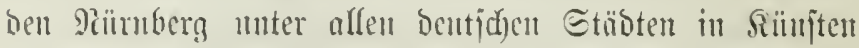

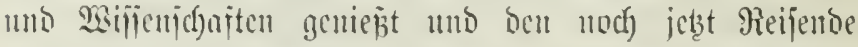

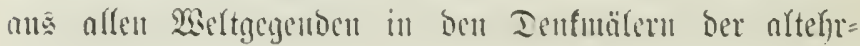
mirbigen Poris betwntobern.

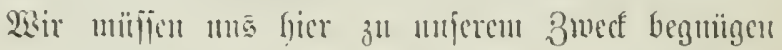

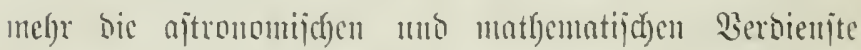

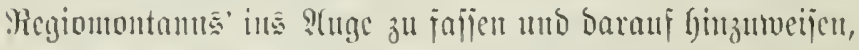

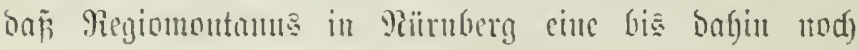
niemals in Ientidstano in joldrent Grabe voryefoumenc

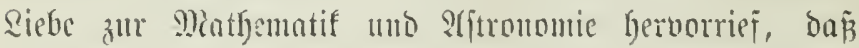

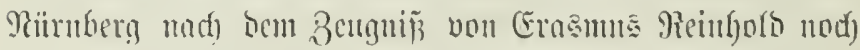

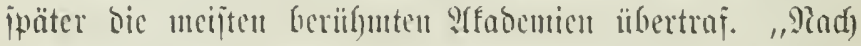

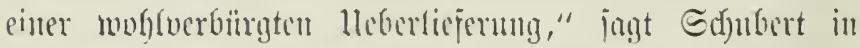

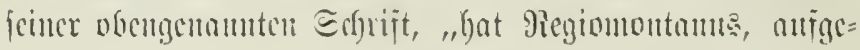

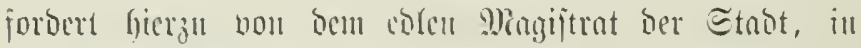
Peïnberg ijfentlicfe Borlejungen ïber Mathentatif unto

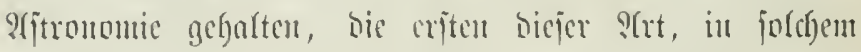

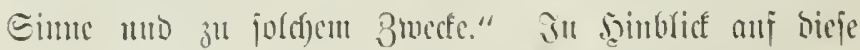

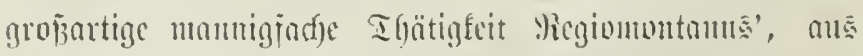

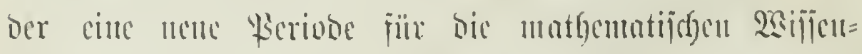

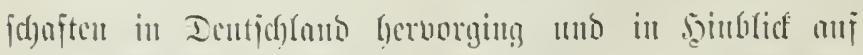

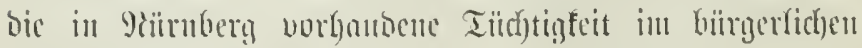

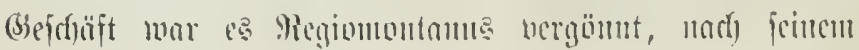




\section{$-17$}

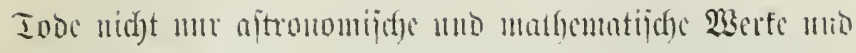

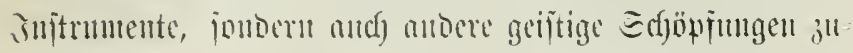

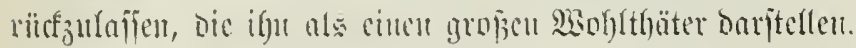

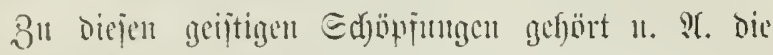

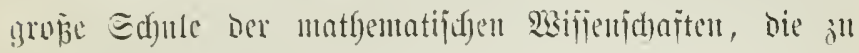

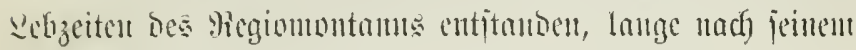

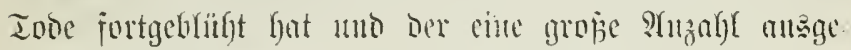

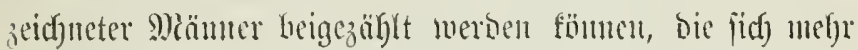
noer minder un bic veridjiebenen 3̈weige ber siftronomic, Mrathematif, S(ritfmetif, Geometrie, Dptif 11. i. M. wer= bient gemadyt hahen. Wian ben hantiädflichiten bieprä= jentunten Diejer Sdjute mögen Gier n. श. genamnt werben:

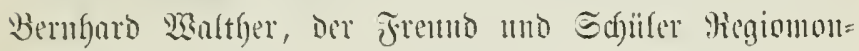
tamıs' (geא. 1430, gejt. 1504), Der jeinen Qef)rer um 30 Jalyre jiberlebte mo ber, werm er and nidyt ben viefjeitigen Geift und bie mamnigfaltigen Remutnific bes

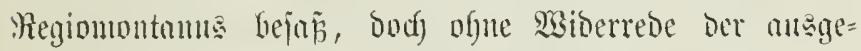

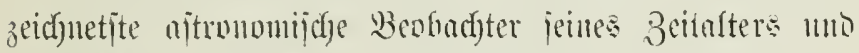

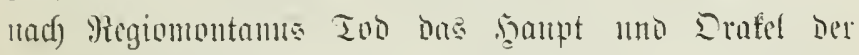

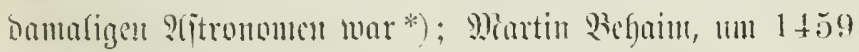

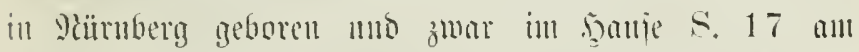

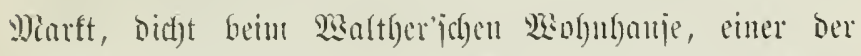

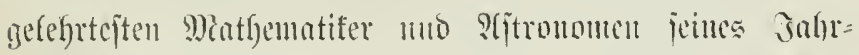

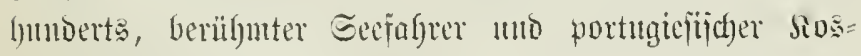
mograph, aud ein Sdjüler bes Regionoutams, gejtorben

*) (E. F. Ifpelt: Die Mieformation ber Sternfunde. ङ. 55 . 
in Sijīabon 1506 ; Joban Esemer (geb. 1468, gejt. 1528), cin guter bimmeloblenfordeter uno vorjüglidjer Genuteter, our 11. ?.

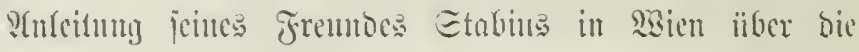

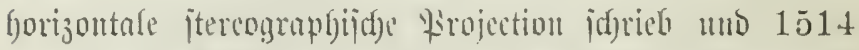

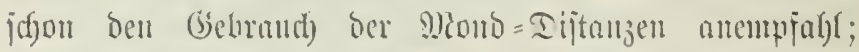

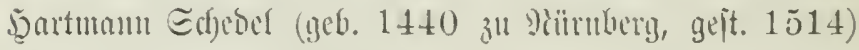
practigirte in jeilur bateritnot, nadfocm $\mathrm{cr}$ in Pabua

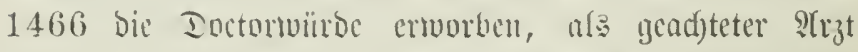
molo laty bjijtorijden uno antiquarighen Etubien oh, von

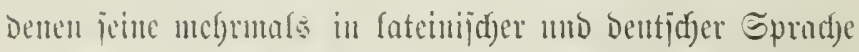

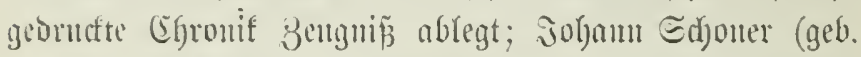
1477 zh Siarljtats it Franfen, gejt. 1547 in Sä̈m=

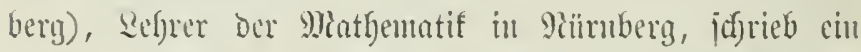

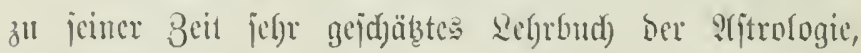

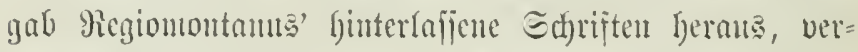

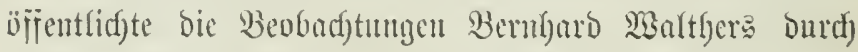
Den Drutf 154.4 mid verjaf̧te bas Opusculum geosraphicum, für sie (bej)idf)te oer Entmicfefung ber geo=

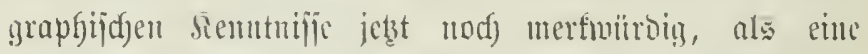

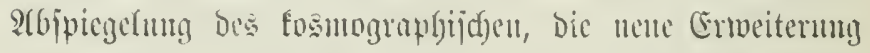

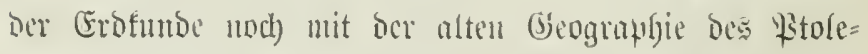

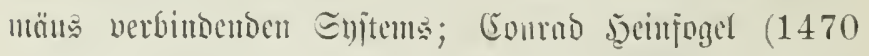

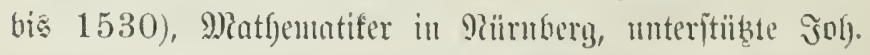
Berner bei Decranggabe ciniger geographijdyer Sacte und entwari nad) Den Boriduriften des Etabins eime Etem= farte, fiir meld)e s(rbsed)t Türer bie Jignuren zeid)mete; 


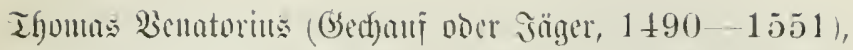

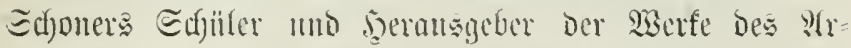

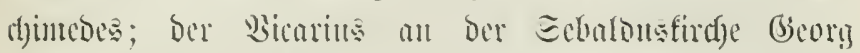

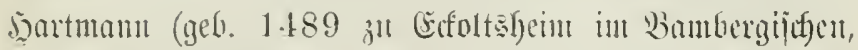

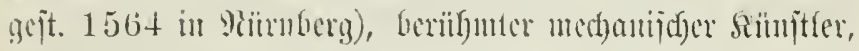

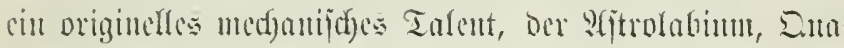

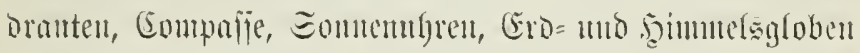

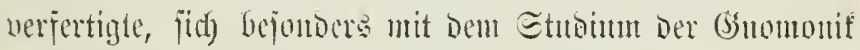

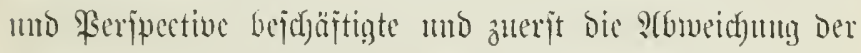

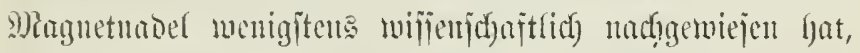
1538 dic Decfination Der Domals in ?) Cümberg $10^{\circ} 15^{\prime}$

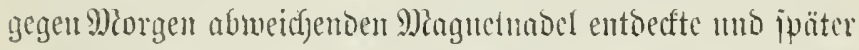

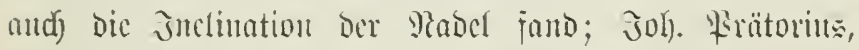

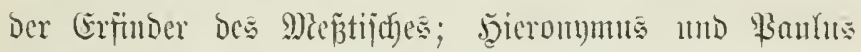

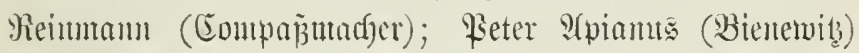
añ Zeisnig in Sachjen, der 1520 bic erjête Randofarte mit

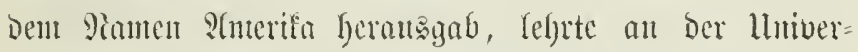

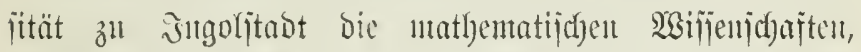

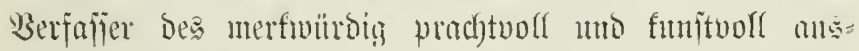
gejtattetell, Astronomicum ('iesareum ", Echülb̨ling

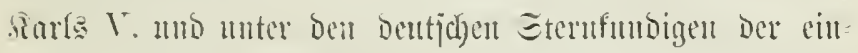
jige, ber nit bent siajer in perjünficher Berbinoung itano;

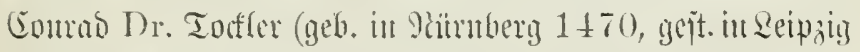
1530); Joh). (5ochlüus (gcb. 1479, gejt. 1532); Errburo

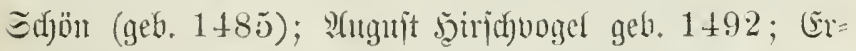

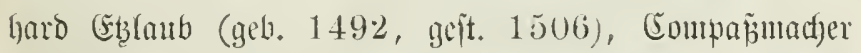
mo Mathomatifer, Berfertiger cinter sarte bon Dentich= 


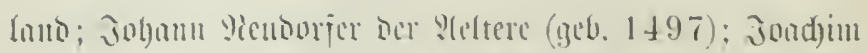
Eamerarins (ych. 1500): bor (volojd)micos, Med)antifex

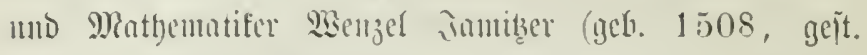

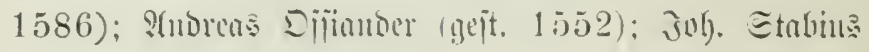

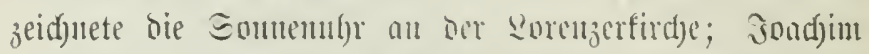

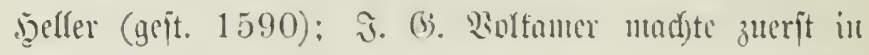
Den Jahyen 167

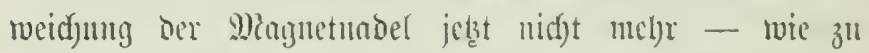

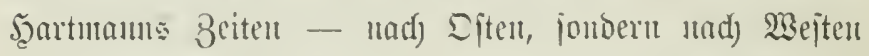

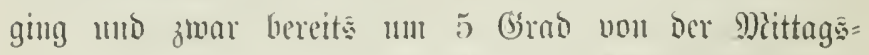

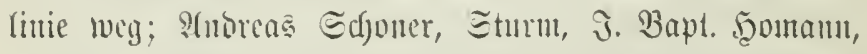
Begrïnoer ocr vormale Griilyenden Eandfartenjammfunty,

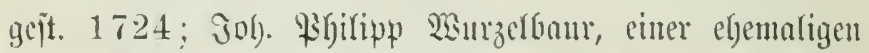

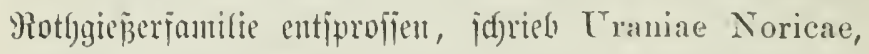
urricftete cinc adflecfige Stermuarte im giofentfal an

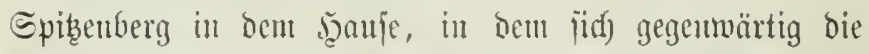

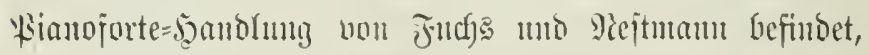
gejt. 1725; Georg Cryriftoph Eimmart, cin geborener Regensuburger, aber jeit jeinem 22. Saljer (1660) in

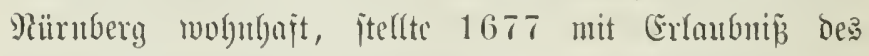

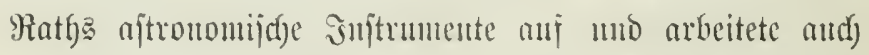

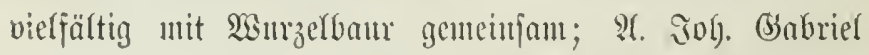
Doppelutand, Ioliag Maner 11. v. M.

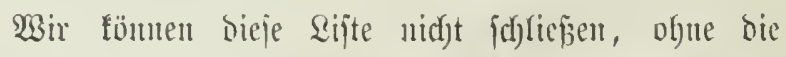

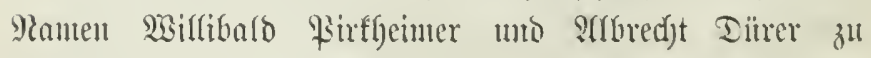
nemnen. Wie Regiontontanus als Der Rejormator Der

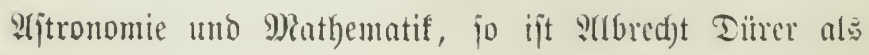


Der Meformator Der Dentichent Maferichule zu Getrad)ten.

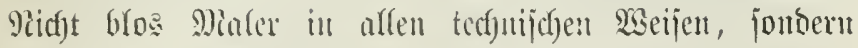
anch) Beidner, Sinpieritedfer, S(eber in Bim uno Eijen,

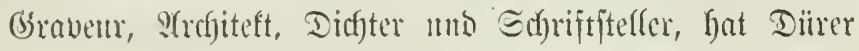
fid) and mittelfor um dic s(jtronumic Berbiculte $\mathrm{cr}=$ morben, intoen or einte Steruffate, wozu ifm der ältert

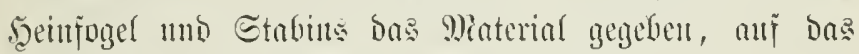

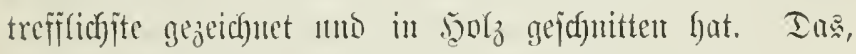
was Pirffeinter (geb. 1470 in Eicfjtäot, gejt. 1530),

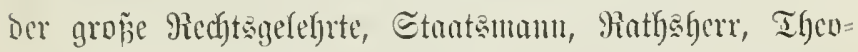

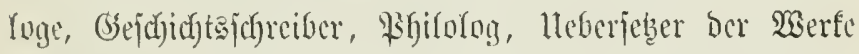

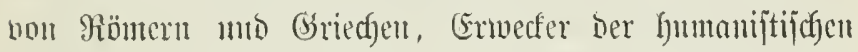
Stmbien, Renuer der D)ïnzen, der Sunnft, Fidjter, antw=

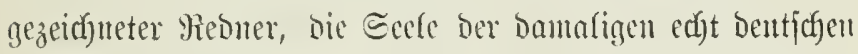

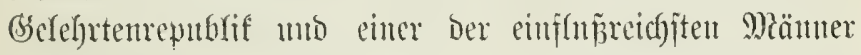
im Beitalter ber Reformation, zur Befüroermung Der ma =

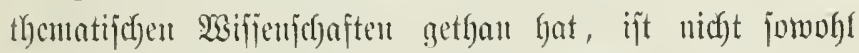

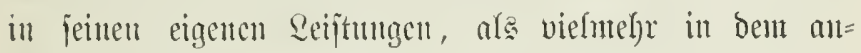

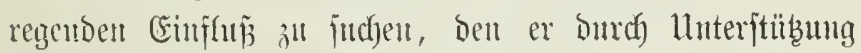
แnd 2(nregung alf bie Miathematifer jeiner Uumgebumg ansgeitbt hat. So gab, wic oben bemerft, Thomas be=

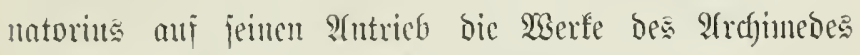
ans Der Firffecimerijden Bibliothef gerans.

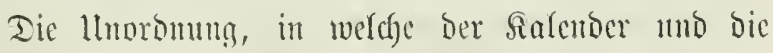

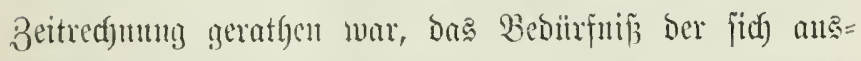

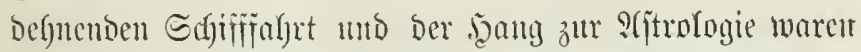
Dem Stubium ber sfitrontontic bejonbers gïnitig unb bic 


\section{$-22-$}

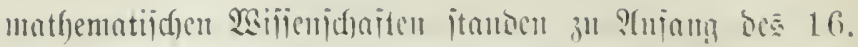
Inf)rfunderts nuch in höherem ?njefen als zu Enoe

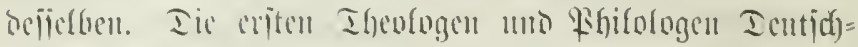

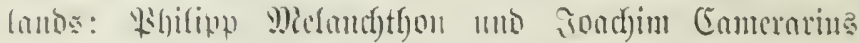

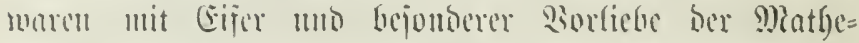

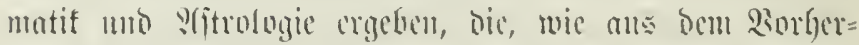

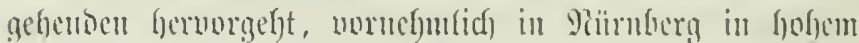

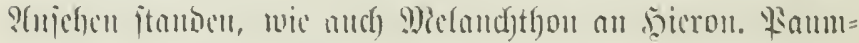

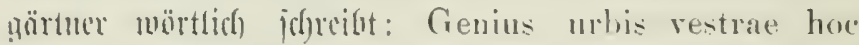
momine veneramlus est ommibns dectis, quod is disciplinis, yuan Mathematicar rocantur, adeo faret, It mequam majoral sint lerum studial, quam in urbe restril, et ingenia ad eas artes aptissima apud ros nascautur etc. Whe all bem Gejagten gefyt herbor,

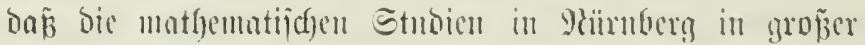
Bliithe itmoen (, in ea urbe studia mathematum

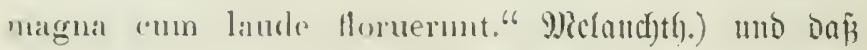

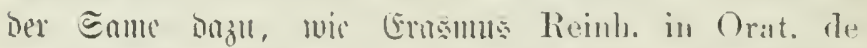
vita Regiom. hemert, unn Shegiomontoms sucrit mit Erjoly angeftrent murbe (.,hujus dectrinale semina

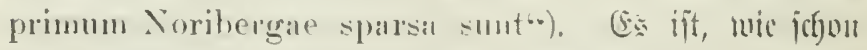

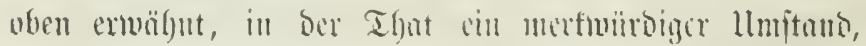

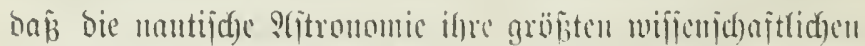

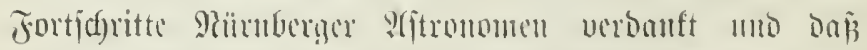

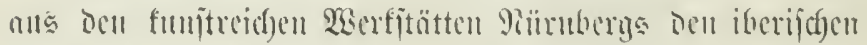
Seefal)rern bie beften nautijden isnjtrumente geficfert murben. S(uch) mag fier nidgt mermähnt hicilion, wic 


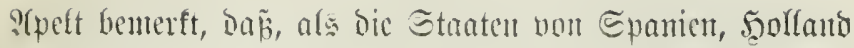

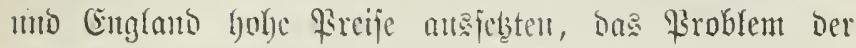

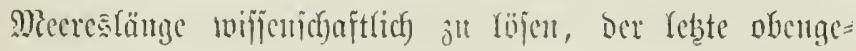

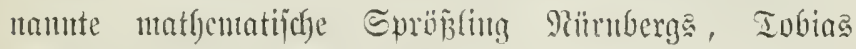

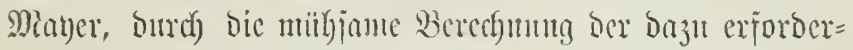

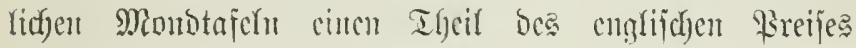
verbiente.

Dic supertigung vou mathentatijech uns aftrono= mijgen Smitrumenten lag Yicgiontontam - mic ans

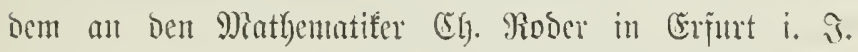
1471 geridyteten Brief johlagento Gerburgeht - bei jeiner

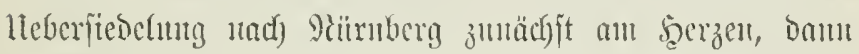

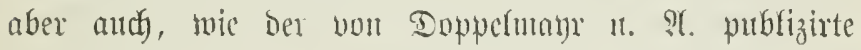
Index operum (,hatec opera fient in oppicio Nuremberga Germaniae ductu Jo. de Monteregio 14736) unt bie in Den Rexicis แntD Bibfingraphien von Roter=

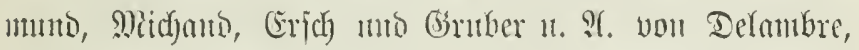
Stern veröfifentfichten Qiften uno 3 erzeichutific ber in

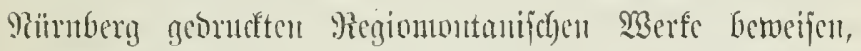
Dex Irutef jeiner in Itatien mb lingaru (Dfen) ge=

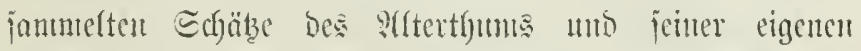

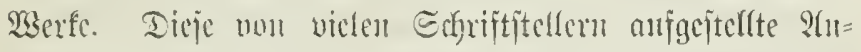

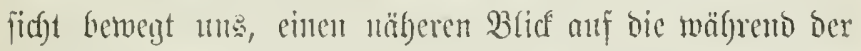

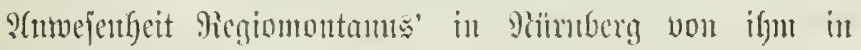
Druff erfyjencuen 2 Eerfi und banu auf bic banale in

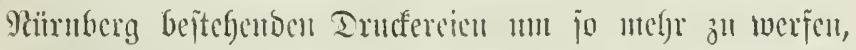
ale vicffach behanptet twirb, baj, ,Dicjen jecros ber gia 


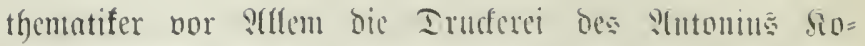

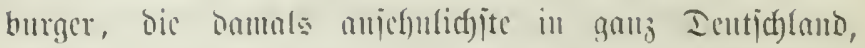

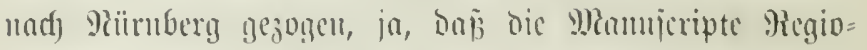
mbutme mit ifren Iajeh แnd nftronmiidgen 3eid)en von ber Bejdyajfenlbeit gemejen, baj ifr. Irudf jelbjt bic

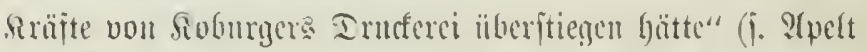

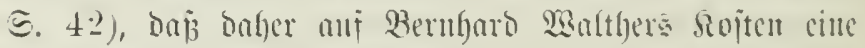
eigenc Intefermerfitütte erridfet morocn, fïr bie Reģio-

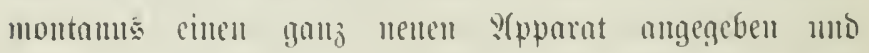
Durdh beficu Erpinoung or ïd) cin gered)tes Berbienit

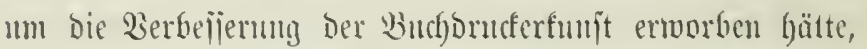

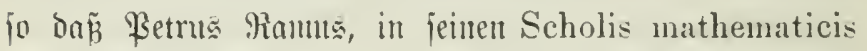
p. 64 feinen 2uitano genonunen, ifn afs eiten Miter= finder (?) Diejer Sumpt zul nemten.

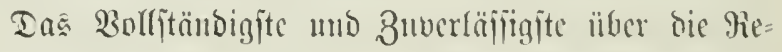
giomontanus=2iteratur findet man, wie fourerft, in oer

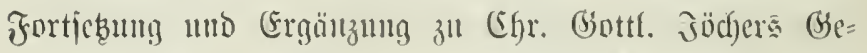
relyrten=2exifon vou .5.

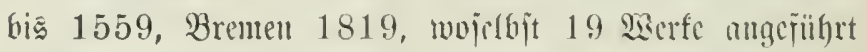
merben; ferner in ber ,Bibliographie Universelle ancien et morlern XXIX (Michand)", wo Delounture

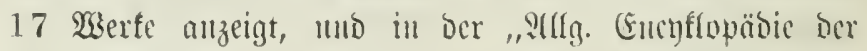

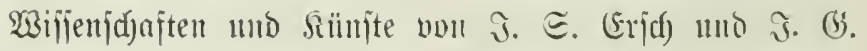
Gruber. Zweite Section. Zeipzin 18+3", wo Gtern unter Der Rubrif: "Johannes de monte regio" 24 in

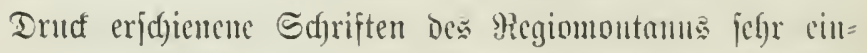
gehend bejpridgt. Wem wir bierzu nod) bic : 


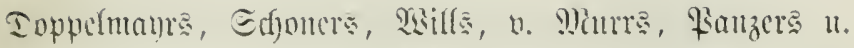

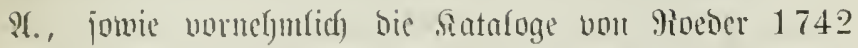

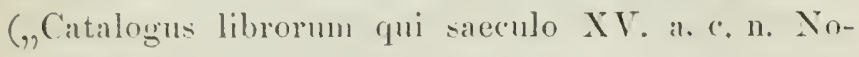
rimbergate impressi sunt collectus ef conscriptus a .To. Parillo Roedero. G. Ae. Rect.") un von 2 secibler (.,Jo. Friederici Weidleri Bibliographia Astronomica temporis, (no libri rel compositi rel editi sunt. Wittenbergae $1755^{66}$ ) redfuen, weldyer letztere 21 a

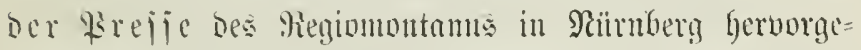
gangene Werfe angibt, jo bïrften bie betr. Enellen

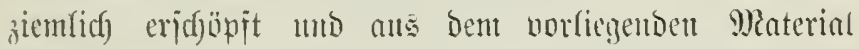
amtäberno Der bejte lleberblisf zn getwimten jein. Sehen

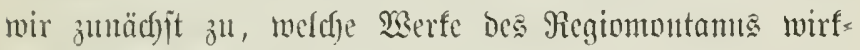
(icf) in Pä̈nberg georneft woroen fint.

P?ach Den chentangefülyrten Dutellen finto ofme Bweifel folgende Merfe bes Reginntontante während feiuer 2ruejengeit in Rïrnberg $(1471-1476)$ gebrufft worben:

1) Theoricae novae planetarmn Ge. Purbachii cum figurationibus opportmis in oppido Nurinberga Germaniae ductu Jo. de Monteregio. Eriffien 1472 ober 1473 (†. Sönthers (sel.= Rer.). Dieje von

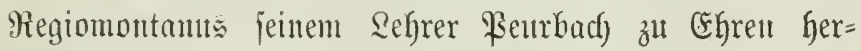
auggegebene S(f)rift wirb audf von Roeder in jeimem Satalog mit Dem 3ujab ductu Joamis de Monteregio aber olye salgregzagl angegcben. Delambre,

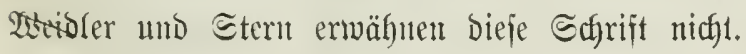


2) Manilii astromonicon ex officina Jo, de Regiomonte habitantis in Nuremberga oppido Crepmanie celchratissimo. Efyc Druffafhr, verumtflidy 1472 noce 1473 ill 4. (i. Söd)ers (Gel.=尺cx.). Ticje Edjuift

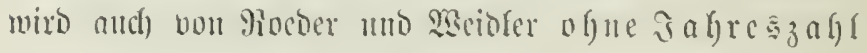
angegeben, vou bem eritgcuanuten mit bem 3ujaks: ex officina de monte regio in Nuremberg. Defanture ıno Etern fïfren bicje Edjrift nidjt ant.

3) Ephemerides, quas vulgo rocant Almanach ad triginta duos ammos futuros. Norimb. ductu Jo.

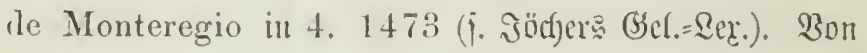

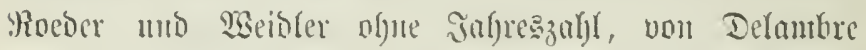
unter Deut Titel angegeben: Ioannis Regiomontani Ephemeride astronomicae ab amo 1475 ad annum 1506. Nuremberg in 40. ?hd vou હteru unter

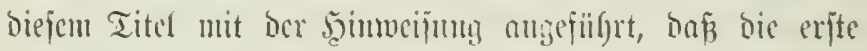

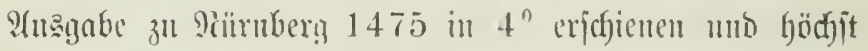
jelten zul jein jufeiue. Maciofer (hist. astron. p. 316) bejd)reibt cin Exemplar nus ber Biblintbef ber Witten= berger affadomic, ocr Titel febll, um Eubs itefen bic Worte explicitum est hoc opur anmo Chr. D) 1474. ductu Joannis de lionteregio. Jod) uermutge auz

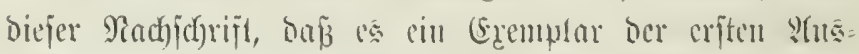
gabe ift, ba and) bie crite Stuggate bes Sulenters bic:

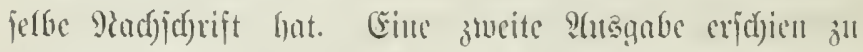

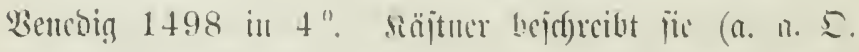

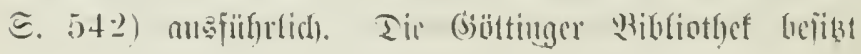




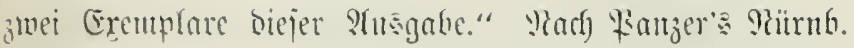
Bufforuferigcidj. p. 166 ïn Ephemerides roelestes 147. 4. ans der eigenen Irufferei bes gegiunton=

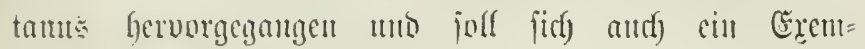
Hlar Der Ephenterioen auf ber 9eiirnberger Biffintgef

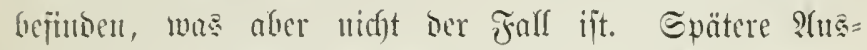

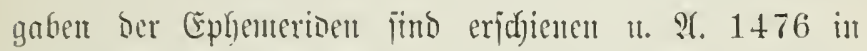
Benedig, 1484 ebembajelbit per Erh. Ratdolt, Ephemericles ab an. 1492-1504. Venet. 1492. 4., Ephemerides accuratissime calculate. Sequitur tabula regionum. Prognostica in compendium redacta. Venet. 1492 impensis et arte Tinc. Benalli in 4., ferter Ephemerides ab an. 1474 ad an. 1506 am Ende 31. Iec. 1506. 4. Dhue Sagr unt Dot; To. de Monteregio - in Ephemerides aut diurnales commentarium. Etelyt in Almanach nova plurimis annis renturis inserventia. Per .Jo. Stoeftlerum . Justingensem et .Iac. Pflaumen, Ulmensem etc.

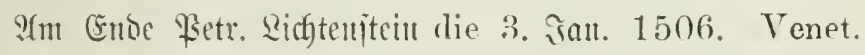
in 4. ih. 1507 . ib. 15213 . 4. Fermer ijt erjojienen: Jo. de Monte Regio: zerman. decoris etatis nostre astronorum priucipis Lphemerides: an Ende foll 8. b. explicitum est hoc opus 1484. 2. Non. April. Venet. in 4. per Erh. Ratdolt mo: Jo. de monte regio germanorum decoris, etatis nostre astronomorum principis Ephemerides itr 4. Dhne Jafr und Ert. Sft in \&eipz. 2 . L. 2 \&. 1798 num. LIV. Col. 


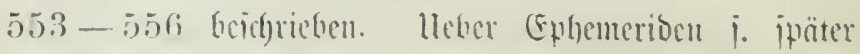
9äherts.

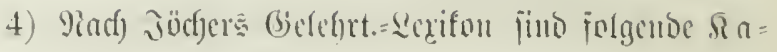
I c noer erfichienen:

a) Callendarium latinum Jo. de Monteregio 1473. 4.

b) Ior teutiche Salember uon 3of. v. Sumgêperg 1473. 4. Er ijt nod) mit in .onlz geidjnittenten ganzen Tajelu geonuct.

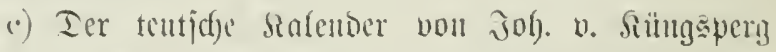
1473. 4. 2lkit bemeglidgen Tajefn gedrudt.

d) 3or. vou Säulperg satenber 1475.4 .

c) Calendiutium novum arl an. 1476. 4. Venet. 1476. Fol. c. Fing. 1482. 83. 84. 85. in 4. Iie Edfrifi gieg. De reformatione calendarii cridjicn Benedig $148 t$.

f) Calendanim, Venet. 1476. Fol. (c. Fig. Erfeint von der vorigen veridjicoen zu jein.

g) Il Calendario di Ciinv. Regio Montano. V'enet. 1476 gr. 4.

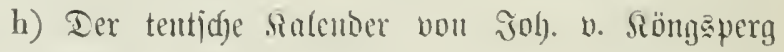
n. Эagr $1+76$ in 4.

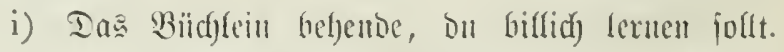

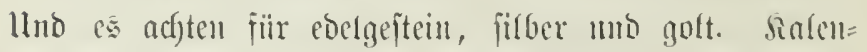

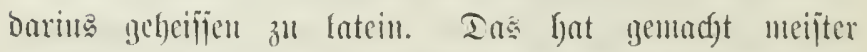

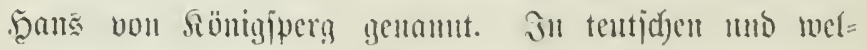

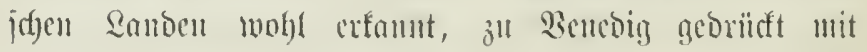




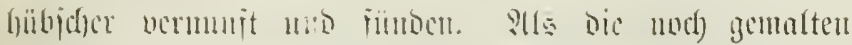
meifter moff fünow $(1+78)$ Bermbart. maler Erfyart

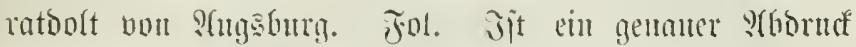
Des tentidgen Safmoer vom gabre 1473.

k) Almanach magistri .To. de Monte Regio ad aunos ITIII accuratissime calculata. Erh. Ratd. Augusten - industria et mira imprimendi arte: qua nuper Veneciis: nunc Auguste vindelic. - tercio ydus Sept. 1488. 4.

1) Calendarium. Venet. 1489. 4. M)aittaire gifot ben sitel an; Io Regiom. de reformatione Calendarii.

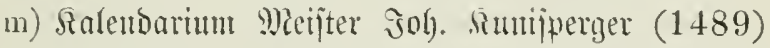

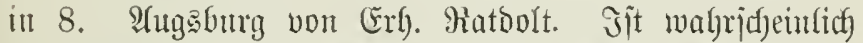

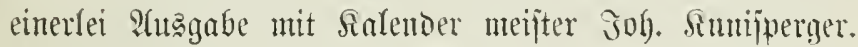
2usgb. von Meifter Erh. Matbort 1489. 4.

n) Salender ?atgăb. von Erb. Ratbort 1496. $t$.

o) CFin falember mitt fünem nümen แnd ftunden us - Jof. funjpergers practic mo junit bil jubtifer jacfen mit viel f̈guren ale man am nädjiten Blatt lïtrer ntel= Dung f̈ndet. Bürid) 1508.4.

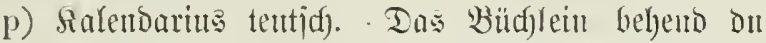
billid) lermen jollt (llno es ad)ten für edelgejtein, jüher ImD golt) Salendarits gebaipen zu latein - von ben 12

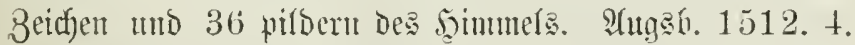

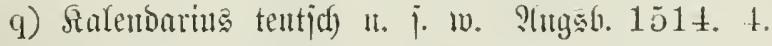

r) Salendarius tentid) น. i. w. :(ugsh. 1518. 4. 


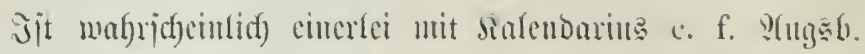
1518. 4.

s) Sïnigiperger Salcmonrits Der 7 Plancten, 12

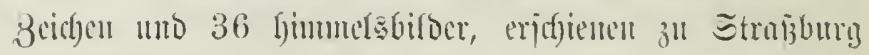
i. วิ. 1535 .

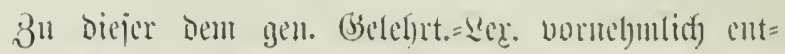

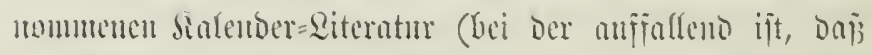

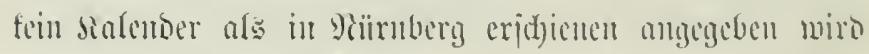

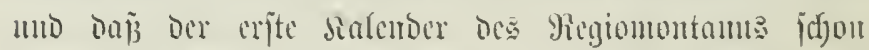

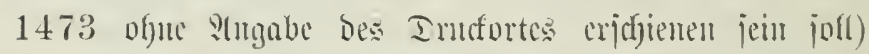

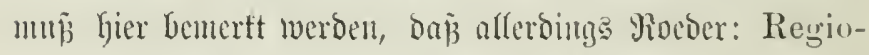
montani Kalendarim novum etc. Nurembergae duetu

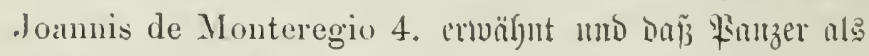
Froducte an Regiomoutami 多rejie nud zuei

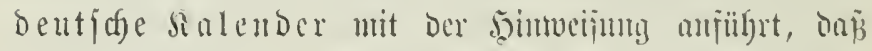

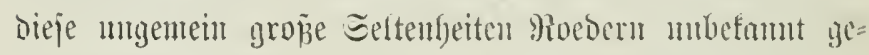
blieken jeient.

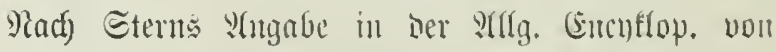

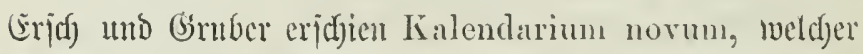

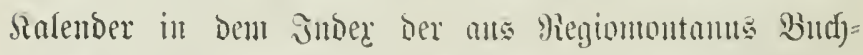
Drufferei gervorgegangenen als jertig antgezeigt miro, zu

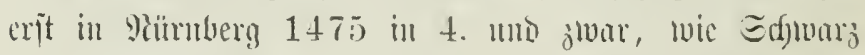
(a. a. D. כ. 63) madyucijt, jull glcidjer 3eit bentid)

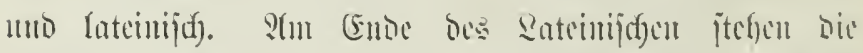
2Borte: ductu Joannis de monte regio, an Eutoc

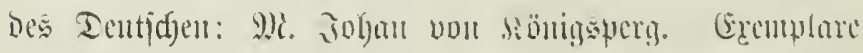

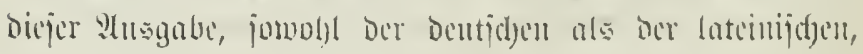




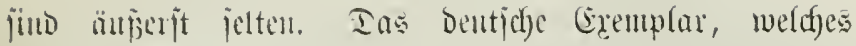

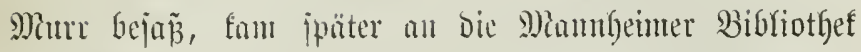
(De Nurr not. tr. codd. p. 20 n. Sigf. autf) Mutre Nemorab. bibl. Norimb. T. I. p. 321).

"Ier Salember," jugt Etern, "ijt mijprïnglich für bie Julgre 1475, 1494, 1513 beftinme, Die In! 19

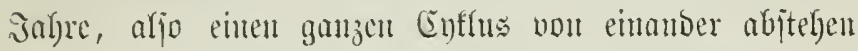

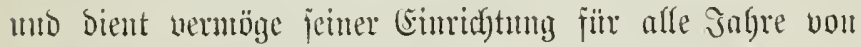

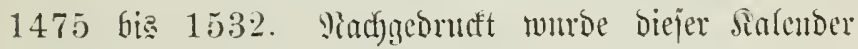

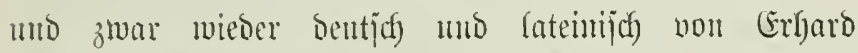

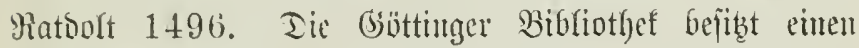

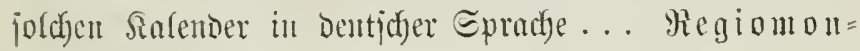
tan's salcuber ijt jedenfalls der erjte, der in Europa georud worded ijt, wem es antíl juon friiffer anbere gejdjriebene gab uno er Gat lange als Majter gebient, jo baz audj die jpäteren sialender, bie ïber bus Jafyr 1531 hinalagelfen, bod) nod) nadh Regionontans Mamen genaunt merten.*)

*) Der 1474 jowohl in Rüruberg al: ill Bentedig in Folio er=

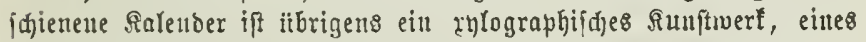

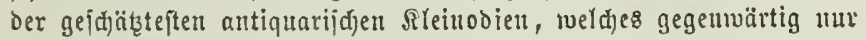
nod) in 5 Exemplaren (wo?). vorganden ntto bei jeinem Erja)eitten mit zuölf ungarifden (sologulden bezaflt worben fein jolf. - Der SaYender if ons nothwendigfie aller Bitd)er uno bas Beourfuin, bie Beit in gewiffe अerioden eiuzutheilen, mujte fehr frith gefiihlt werden.

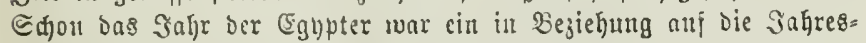
seiten beweglidjes mit bem Rondlanf in feinem Bujammenfang fehendes Sommenjahr von 365 Ingent, getheilt in 12 Monate bou

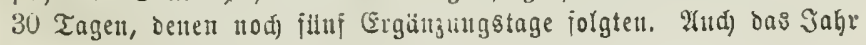


:)(udd) Ectambre bejoreibt Kalendarimu normm.

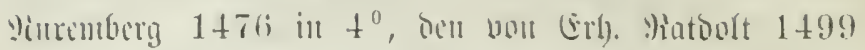

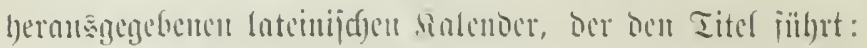
Kalendarimu magistri Joannis de Monte Regin viri peritissini. Ier und) Stern 1485 (?) in Wencoig or=

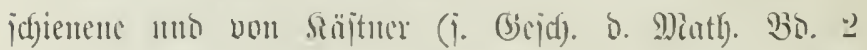

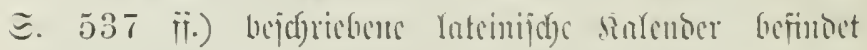

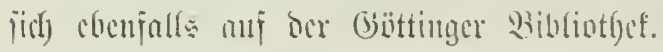

5) Dialogus contra Gerh. ('remonensis in Platnetarum theoricas deliramenta. Nor. 1475. Fol.

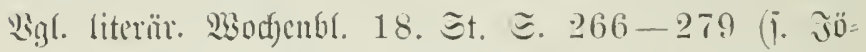

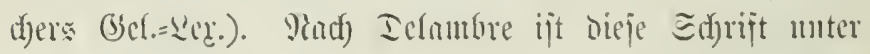
Dent Titel Disputationes contral ete. in ?lïrnberiz $1+74$ uno Ipüter in Bentedig arjejiente. Etefhen it Jo. de Sacro Busto opusculum Sphatericmn Impr. (Venet.) Wrh. Ratdolt 2. Nor. Inl, 1484. 4. ihid. 1485.

ber Suben, (Bried)en แ1. 2. beftans fajon in ben alteften Beiten, wie nod) gegenwärtig bei ben Snden, aus 12 Mionomonaten. Don Sntius

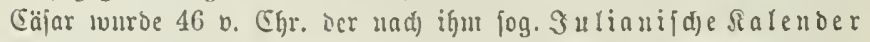

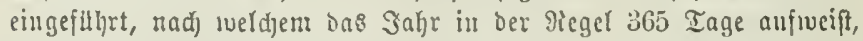

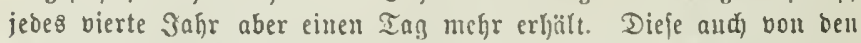
Egriften ofne Aenderung angenommene Sultianifde Einfdultunge. methode ift über 1600 Jahre beibehalten worden, bis Papft (5regor XIII. in 2 fuitrage bes Tribentiuer Concili.ms eine genanere eins führte, bie unter bem Mamen bes (5) regorianijaen Ralenders befant ift. Dor Gulianijale stalender ift ur bon oen Fiufien uno \#berhaupt von ben Befennern ber midt mirten gried. Rirdje beibe= halten worben, weshalb fie binter oer ilfrigeu siselt (jeit 1800) um 12 Tage zurilfgeblieben fino, bie fid) 1900 anf 13,2160 auj 14 Tage bermegren twerten. 


\section{$-33$}

Venet. 1488. 90. 91. in 4. in Sphaerae tract. Jo. de Sacrobusto. Venet. 1531. Fol. S(ud) nad) Stern ijt Dieje Edfrift unter Dem Titel: Disputationes contral

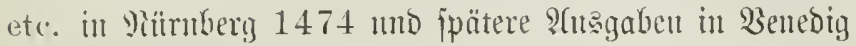
1496, Fol, Damt Bajü 1543 und 9?orimb. 1530 unter bent Titel: In Ptolemaei magnam constructionem, quan Almagestum vocant, libri tredecim conscripti a Joanne Regiomontano erffjienten. Die Phïrnberger Stabtbibliothef bejiz̧t (?) cin Erentplar ber erften Saggabe. Die tabula fecunda muñ Regiomon= tant: bei Stusarbeitung biefer Sdjrift nod) nidjt gefannt haben, rwie Delumbre (hist. de l'astr. dn moyen àge p. 285) bemerft.

6) To. Regiomontani Index operum, ber eigent= liche Titel ift: haec opera fient in oppido Nuremherga Crermaniae ductu Jo. de Monteregio 1473. Fol. pat. 2(unf Der erften Colnmme fund bie Ecfriften amberer, auj ber zweiten fene eigenen angezeigt, bie er

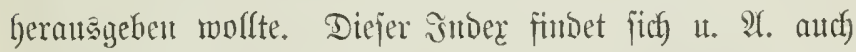

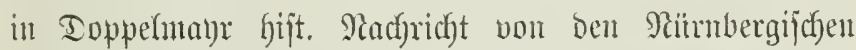
Minthematicis $\subseteq .23$ mo in Panzers ,A Annales Typograph." Tol. II p. 233 abgeorucft. Regiomontants verïfifentlicfte bicjen siatalog bon vielen anderen noch vou ifm zu crwartenden mathematijchen Werfen und fulfr, menn jich andf) viele ber belefrten, Denen biejer Siatalog zur Begutachtung vorgelegt worben, ungiumtig Dartiber ansgejprodjen, mit bem Drulfen weiter fort. 
Radf) Dem Tode des Regionontants, bemerft Toppel= mayr S. 12 , funs bie melyrjten in jeitem ferausgege=

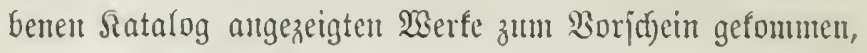
wie eben biejer Satalog bemicje, Der von Regiomontum in zmei Theile gejondert jei. Der erjte Theil gibt bie Titel bon 20 Serfen an, meijt $\mathfrak{B e r f e}$ verjuiedener überjetzer oder verbefjerter alten Miathematifer. Der zweite Theil weift 22 9'umnern auf und gift von ben= jentigen Werfen Madfridft, weld)e Regionnontants bei

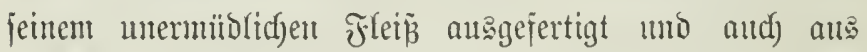

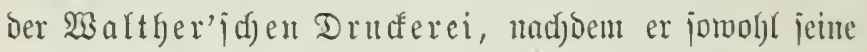
Ephemeriben als cinen Salentoer, vermöge jold)er allbereit bejördert, flt ebiren im Siml gebabt.

7) Tabulae directionum, Nor. 1475. Fol. Venet. 1524. Fol. Tübing. 1554. Witteb. 1606. 4.

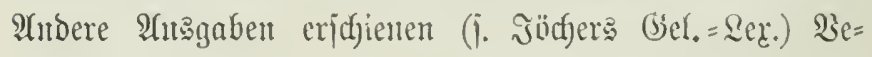
medig 1485. 4; fermer Tabulate directionum profectionumque in nativitatibus multum utiles. Aug. Vind. 4. Non. Jan. 1494. Fol, eine andere ?lusgabe Excud. Ulr. Morhard, Tübingae 1530. 4., in 9üirn= berg ohne Jahr in 4., Venet. 1504 per Erasm. Reinholdum Salveldensem supputatae Witteb. 1584.

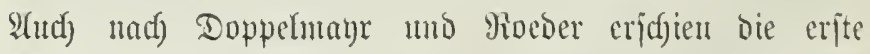

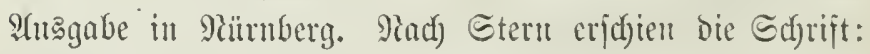
Tabulae directionum, profectionumque zuerjt unter Dem Titel: Joh. de Regiomonde Ludus Pannoniensis, quem alias vocare libuit Tabulas Directionum (No- 
rimb. 1475.4). Sie ijt bejonders zu ajtrologijfen 3 weffen bejtinment mo in Dien für den Erazhiffdof Gran, Der ein Siebraber ber slitrologie war, gefdrieben utno murroe nodf bor Riegiomontans gicife nach Rom in Rïrn= berg gebrufft (vergl. Jac. Aug. Thuani Hist. Lib. 90

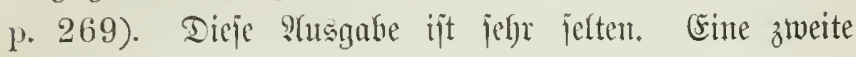
ebenfalle jeltene Salugabe hat bie Mradjidyrift: Opus tabularum directionum profectionumque .... per magistrum .Joannem de Regiomonte compositarum Anno Dei 1467 explicit feliciter Magistri Joannis angeli viri peritissimi diligenti correctione. Erhardique Ratdolt mira imprimendi arte: qua nuper Venetiis nunc Auguste Vindelicorum excellit nominatissimus, 4. nonas Januarii 1490. 4. Ŷtndere Ánggaben: Venet. 1524, August. Vindel 1552, auf Dem IitefGrait heipít es tabulae .... non tam astrologiae judiciariae quam tabulis et instrumentis astronomicis rariis conficiendis plurimum utiles (Tnbing 1554, Wittenb. 1606). In Der Bibliographie

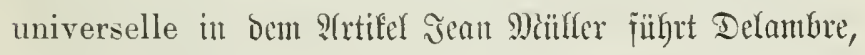

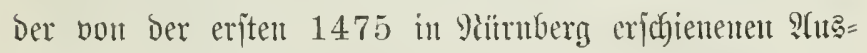

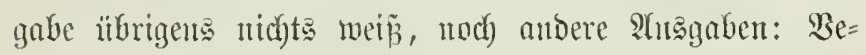
mesig 1485, abgebructi 1490, 1504 mit einer Siluts: tajel, 1550 mit einer Simntajer für bie einzeftent Mi=

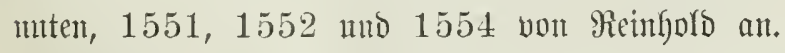

3n Der Borrebe nenut finf ber Berfaijer Johannes

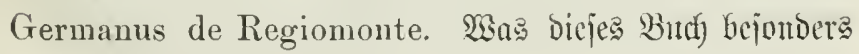


anseicfuet, ift bie tabula fecund:1, D. h. nad) unierer Epradje ente Iangententajer, weil jie bie erjte ijt, von ber man mit Bejtimmtheit weirj, baj jie in Europa bered)net worben ift. S(bor in bem llmjtanoc, ba je fie

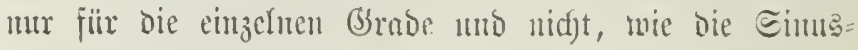
tajel, fïr bie einzelnen Mkinuten beted)uet ijt, liegt and)

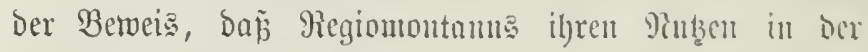

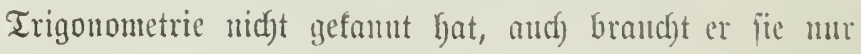
ale Şürf̧stafel bei einem ipeciellen Jalle, wälreno bie Traber jajon 500 safure frither Tangententafiln hatten

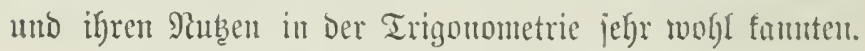

8) Tabula magna primi mobilis cum usu nultiplici rationibusque. Norimb. 1475. 4. 9(ut) vou

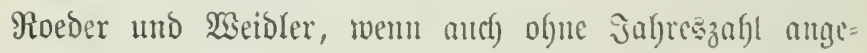
füfrt. Sิm Index auctorum wiro Regiomontamis als Berfajier bezeidnnet. Tabuli primi mobilis Jo de Monte regio. Steht in Tabulis Eclypsium Magistri Georgii Peurbachii. Tiennae Austriae 1514. Fol. 2udf) Delambre filifrt ofiges Sert als i. Э. 1475 in Sürnbery erjofienen an un jagt, Dã Tabulae ecclipsium Purhachii, Tabula mobilis a Monteregio. Venise

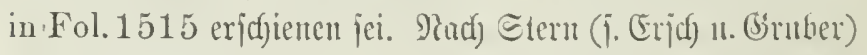

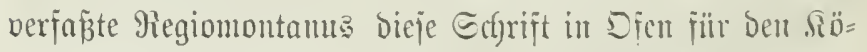
nig Mattfia Corvimn. Eic fan zuerit nebjt anderen

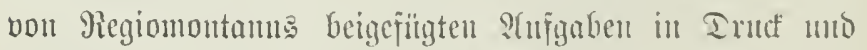
eriffien zuerjt in stumberg (1475?) in 4. Eie entrält

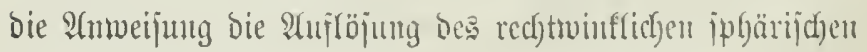


Dreicfs: und Davou abhängentor Fragen, wie bie Dechi=

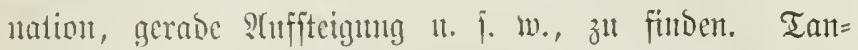
jtetter hat iie mebijt Remrbacje tabulale eclipsium 1514 3n Wien herntagegeben, baun Echoner in Nenburg an

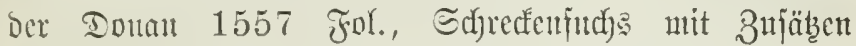
(Bajil 1567) Fol. Dazu gebört Fundamenta operationum, quae fiunt per tabulam generalem Neuburg 1557. Fol. Dieje Tafeln finto eben fo wie bie Tabulae Directionum und bie Epheneriben volf fiföpfe=

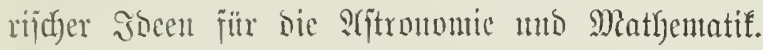

Sou bicfun angefilfyrten Sanptworfen Dee Regid=

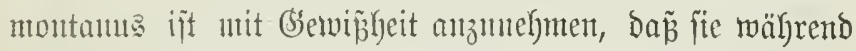

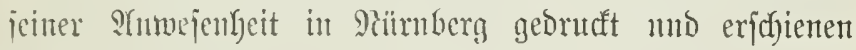
fint und Daj Demuach) biejer Stabt bie Eryre zufommt,

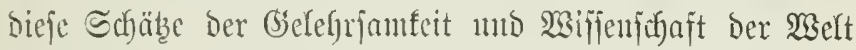
zunüchjt mitgetheilt jut Gahen. Die ïbereinjtintmentoen Entelfentangaben iprechen cviosent bafït. Bei ber grofien

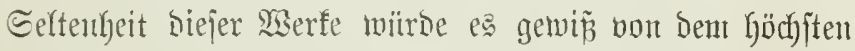
snterefic jein, zu erfalyren, in weldyen Biblinthefen bie

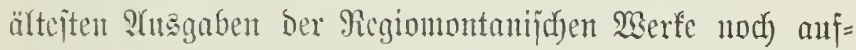

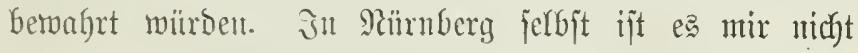
gelungen, bie meltberiifunten Ephenteriben und ben $\mathfrak{I} a=$

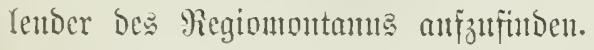

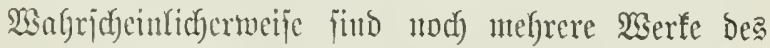
Regiontontants, añ̃er Den angeriifgrten, wäfrent ber $\mathfrak{A} \mathfrak{n}=$

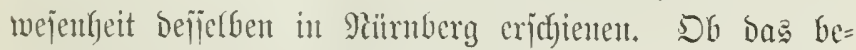
ritfmte $\mathfrak{W e r f}:$ De cometae magnitudine etc. in Nïrn= 


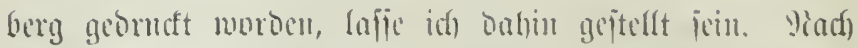

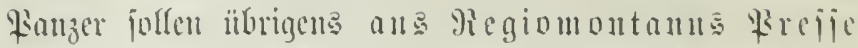
and) bic Edfriften Maffei Vegii Philalethes in Enturto (jaturijdecs Gocbidft) umb ,Magni Basilii Liber ad Jurenes quantum ex gentimm libris ad litteras proficiant in Quarto herungrgegnengen jein.

Es entiteht mu bie interejiante Frage, in meld)er

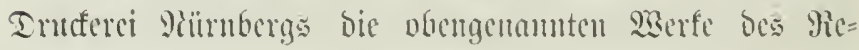
giomontanu wäfrento jeiner s(nuejenfeil in bicjer Etabt (1471 bis 1475) georufft morben jein mögen. In bem

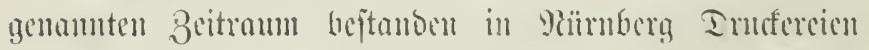

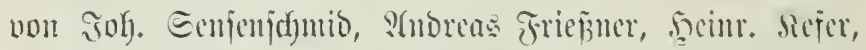

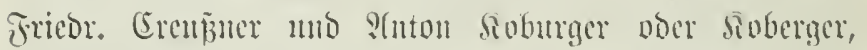
medche 9)iämner bie 1479 andf) bic cinzigen gerwejen, bic

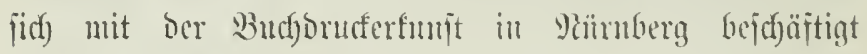

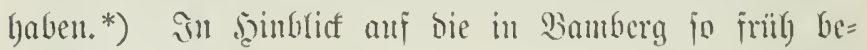

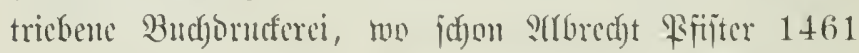
unb 1462 georuteft, ja jugar żvijdyen 1453 mo 1455 eine ganze lateinijacte Bibel zu Etanoe geforad)t

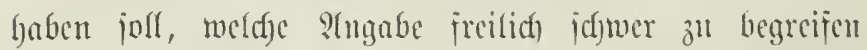
ijt, Da in Mlainz 1455 noer 1456 , madj antoren Satgaben 1450, Gntenberge crites grojes Truffucrf,

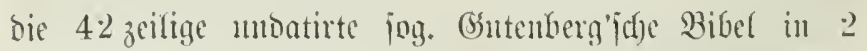

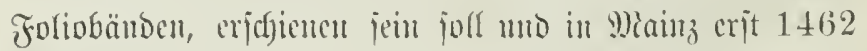

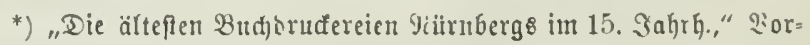
trag, gehaltent dajelbft int Riterarifdyeit Berein am 20. Plärz 1873. Eergl. Sorrefp. v. 1t. f. D. D. 22. MRär 1873. 
Die ganze lateinijhe Bither fertig murbe, ift es auffallemo,

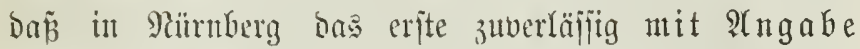
Der Beit uto des Druffortes (aber nicht ber Drufferei) georucfte Butc): „Francisci de Retza. Comestorium vitiorum" (meldfer fitriojer Titel vielfeicht mit "Epeijejaal Der ¿ajter" zu itberjeben jein bitrfte) erjt 1470 erjojeint, werdyes nad) Pantzers Forjafungent unzweifelfaft ans Senjenjajutios Prejle gefommen, ben man aljo mit Hedft den erjten Päruberger Inuffer menten fönnte. Bor bent Jahre 1470 ijt bennach in Rïrnberg fein B̉uch gedrudt worden. Tas 1472 erjofienene Werf des gelefyrten Iontherrn arfbert v. Enf

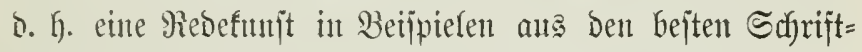
itellern, ijt aber bas erjte mit Senjenfadmios ?amen und

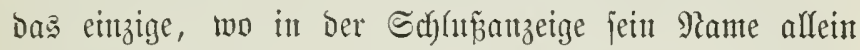
vorfommt. Das im Jahre 1472 in গitrnberg erjujenene Buch „Alcinoi disciplinarum Platonis epitoma" hält man fïr bas erite Bucf), das aus 2 (ntou Soburgers Brefie mit ber Snzeige Des Druffortes, aber ohne Pamen gefoumen ijt. S(ls bas erite Budf), Das Soburger mit

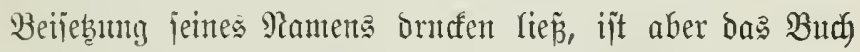
Des Boethits zu betrachten, das int Jafre 1473 eridfienen

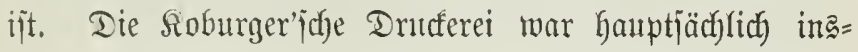
bejoutbere Dutch bie präcthtigen uno johnell auf einander folgentoen bentichen uno Yateinijdyen 24ägaben ber Bibel berïfmt. Die erjte it Mü̈nberg gebruffte Yateinifde

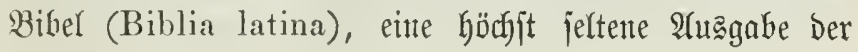


Bulgata, ijt wou ?fut. Sinkurger 1475 gedrufft worben. Das erfte filmptlerija) reid) als Gurgers firefie ift aber bie bentide Bibel von 1483. Im Gianzen fino bet ben siofurigern $(1472-1540)$ : 28 la= teiniface, 1 bentjuje แno 1 böbmijaje, zujanmen 30 Bibel= ausgaken gebutuft worben. Iie Piadjpiger bes i. \$. 1513

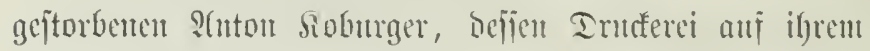
5äbepunt 24 \&rejien gelfabt, an benen 100 Perjomen

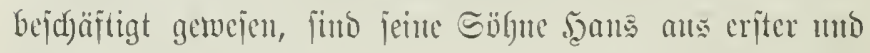

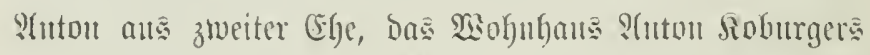

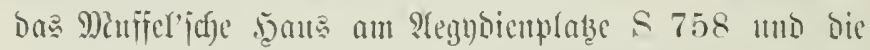
Dondferei, wie audf Serr Dr. Lodfuer vermuthet, in ber R3urgiträ́ S 529, ber હtabtbibliotbef gegenïber, gewejen.

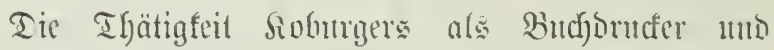

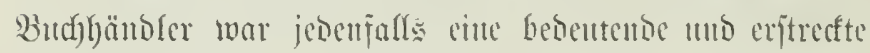
(id) anf bie gejamunte bamalige gelefrte 2 selt. Bon aflen

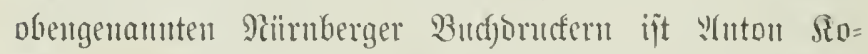
Eurger in ?ajefung ber wablen Berbiente mu bie Bud $=$

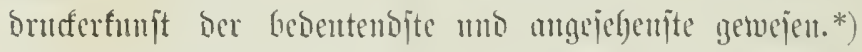

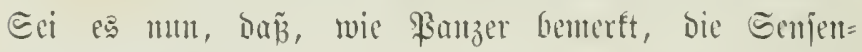
j(f)mib jage Inteferei nicht eingericftet gewejen, um bie

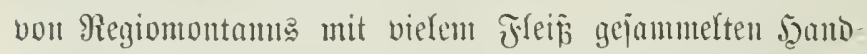
idyriften griechiface und anberer alten matbematijacu

*) Rergl. "Die Sloburger, Bud)gänbler = Familie ju Yiurnberg. (Eine Darftellng des beutid)en Budhandels in ber Beit bes llebers gangs von ber jđolajtijd)en Rififen[d]aft jur gieformation von D\&car .̧aje. Seipzig, Breitfopf uno Fertel. 1869." 


\section{$-41$}

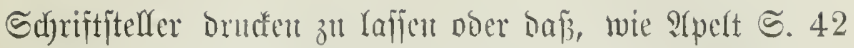
antimut, bie Danufferipte Regiomontans mit ifren $\mathfrak{I} a=$

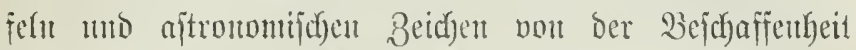
gewejen, Dafi ifr Drutf bie Sräfte ferbjt von Soburgers Drufferei überftiegen uno Soburger Den Druff abgelef)nt babe, was um jo wafrifheintidyer jei, als man bamals (wic ans den Disputationes Joh. de Regiomonte contra Gerhardi Cremonensis in Planetarum Theoricas deliramenta in Folio hervorgehe) nodf nidjt mit

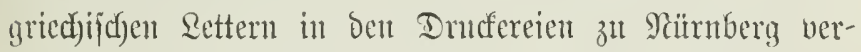
fefent war unto Reginnontan in affe Eremplare ver= jafiebene barin vorfonuntento griedjijche Wärter in bie zmijact Den lateinijacten leer gelajienten Spatien mit eigener .̧anto gejd)riebent habe, wie bergleid)en ?(nzgafen anf ber Shïmberger Stabtbiblinthef Gemeifen (j. Dopper=

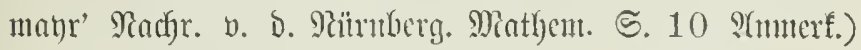
- foviel jteldt fejt, wie aud aus bent Satalogen won

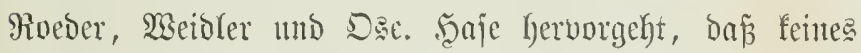

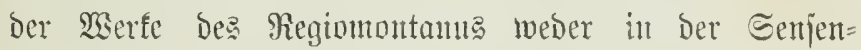

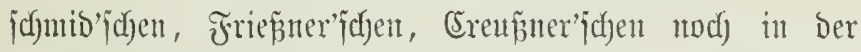
Soburgerijgen Drufferei zum Dutut bejürocrt sworben ift.

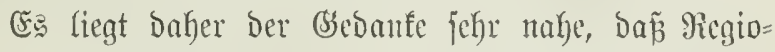

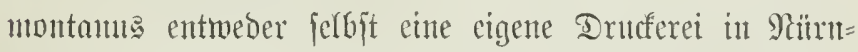
berg gehabt hat poer jeine Werfe in ber Drufferei Des ifn befrentudeten Berulfard Walther, der nadj bielfachen ?(n= gaben auj jeinte Roften eine Druffermerkftätte erridjtet hat, bruten lię́. Der vorfommento Âs̆orud ex 
officina de monte regio, ats Regiomontans Irufferei (i. Hoeber und Panzer) bentet Darani hiu, baj Megin= montante in ber That cine Intferci in Mïrnluerg be= icfien lyake. Sou Picyioutnutane Drufferci, dic er

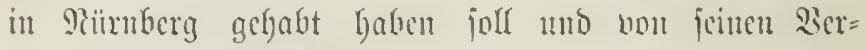

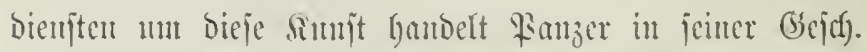

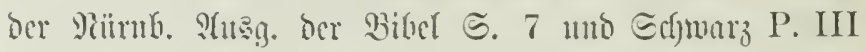
documentos. typograph. S. 46 1. i. S(tud) faum man

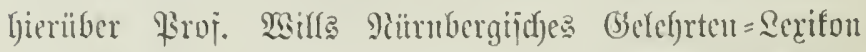

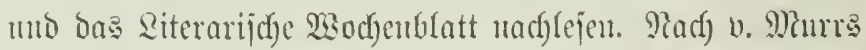

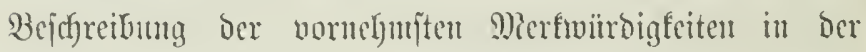

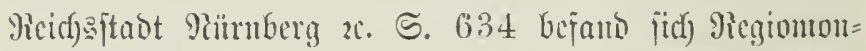

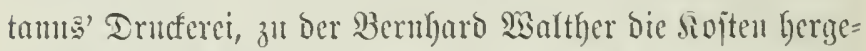

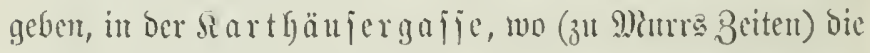

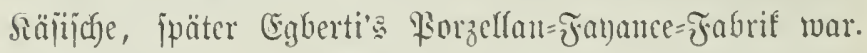

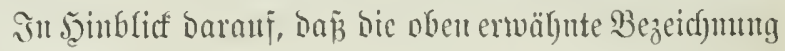
„ex officina de monte regio" mur cimmal, utto zmar,

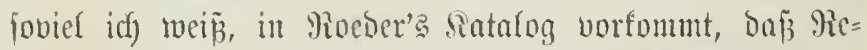
giontontamt: bei feiner vorïfergethenten Sumcjenteit in

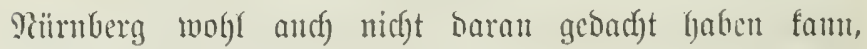
cine cigene mit grojen Geldopfern verbmtone Duncferei

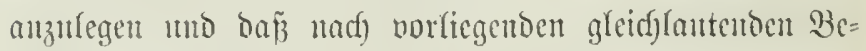

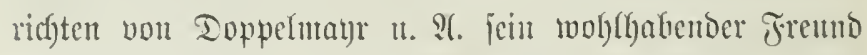
Bernfard Salther auj eigene Siojten in snterelie des Regionontams eine Drutcerei crrichtet hat, fo ijt wohl

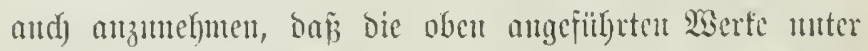
Der Seitung uto S(uffirfft (ductu Joannis de Monte- 
regio) in biejer SEaltger'juen Inteferei hergejtellt worden

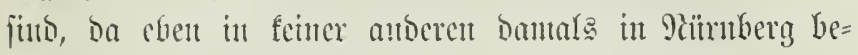
Itebentoen Intuferei, twic wir oben gezeigt, bie in Neitrn= ferg cridjienenten siserfe bes Regiontontants gebuteft morben jins. Späteren Forjdyutgen muj es ïberlajien

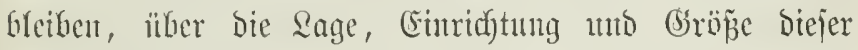

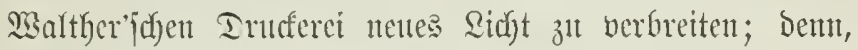

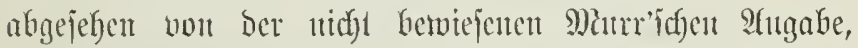

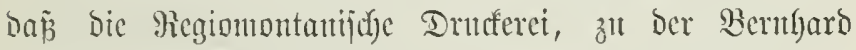
2isaltfer bie sojten hergegeben, in ser Sarthäujergafie

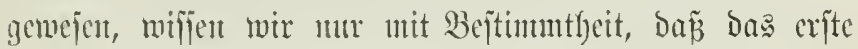
von Sebals Fren), eittem Dhm S(lfrect)t Dürers, gefaufte

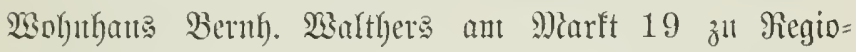

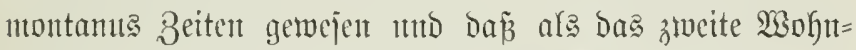

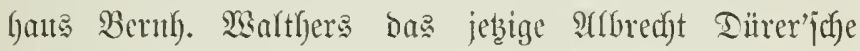

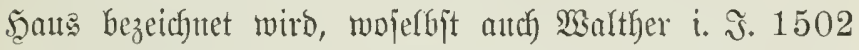
(futrze Zeit bor jeinem i. Э. 1504 erfolgten Tode) mit

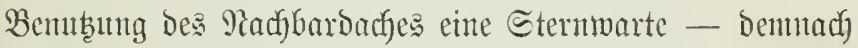
bie źmeite von Waltfer lange nadi) Regiontontanus Tobe gebaute Sternwarte - erridgtet hat, ein Bemeis,

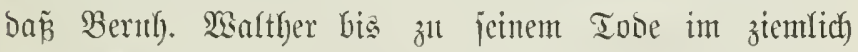

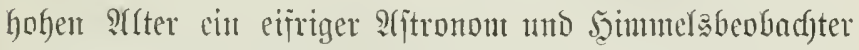
gebliebert ift. Smmerfin breibt es merfwiltoig unt auf=

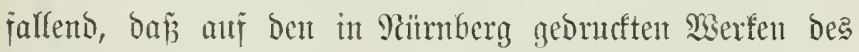

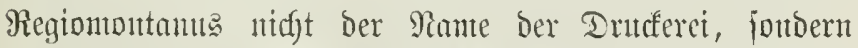
mur b̈fter ductu Joannis de Monteregio angegeben ijt.

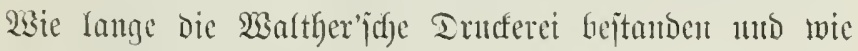


viele siserte als berfelben hervorgegangen, bariber liegen

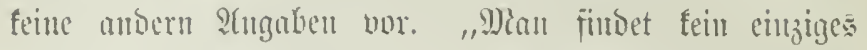
3ud)," jagt Ioppefmatr, "Das 2 alther von Regionon= tauts nad) befien Tob zum Imnd bejürbert bätte (afjo nad) 1476), baber wolje glanblidj, baj jence jeinc Dructerei gar bald, wegen viclen bieriiber gehabten $u_{n=}$ muthes cinum Sudforuffer verfonit ljat."

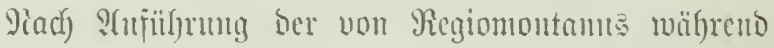

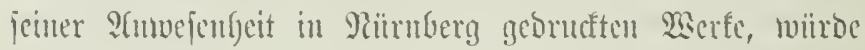

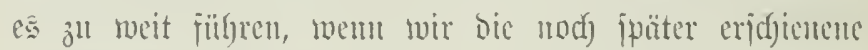
Regionuntume = Siteratur fojer ausfülyrlidjer bcipred)en

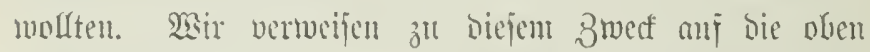

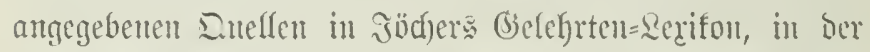
Bibliographic miverselle (Michand), in Der (Enetflopäbic

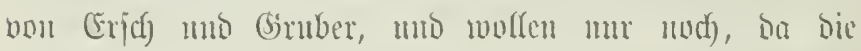
Siteratur Ḧber gegiomontanız, fovid แn hefannt, feinerlei

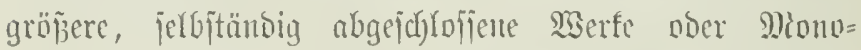
graplien umifapt, ani jolgende Edfriften bi zu

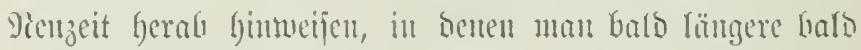

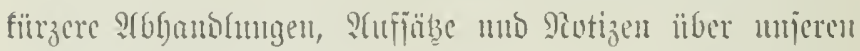
berïfmten Sandsmann finten wirs.

Joly. Gdyoner in jeinen opusculis geogr. Norint. 1533. cap. 2. scripta regiomontani.

Toh. Edjoner: Joh. de monte regio, de cometie ungnitudine ete. problemata XVI. Norimb. 1544.

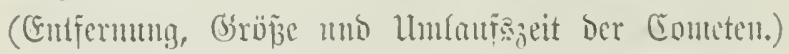

Joh. Schoners scripta clarissimi Mathematici 


\section{$-45-$}

M. Joannis Regiomontani. (Hegiomontamus befauptete lantge vor Sioperuifus dic Betwegntur Der Eroce unt die Somme.)

Erasmus Reinhold in Phil. Melanchth. orat. select. T. III. p. 503.

Erasmu: Reinholdus oratio de .Toh. Regiomontano, gefyalten zu Sittenber! 1549.

Georgii Penrbachii et Joamnis Mülleri Regiomontani vita in Petri Gassendi Miscellaneis. Tom. V. p. 519 (Lugd. 1658) แtmo n. Sifjautb p. 38.

Discursus von bem Juffre 1588 แ. \%. w., enth). biogr. Potizen ïber Regionnontame. 1684.

Petrus Ramus in jeinen scholis mathematicis

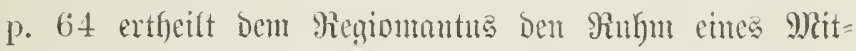
erfinders Der Budforucferfunft. Bergl. ๔. 24.

Joh. Trithemii catalog illust, vir. p. 163 men.

Panl Jovii elog. viror. erudit, p. 218 (Jieg.

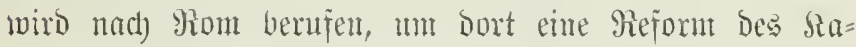
Yentor: zut begrïndent).

Werners Lorredo 311 Georgii Amiercei opusc. gुeogl'.

Mieldi): Actam., vit. pliilosophr. p. 16 (Miingerg

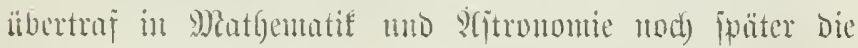

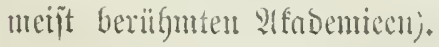

Jaluricin, hibliotheca IV, 353-361.

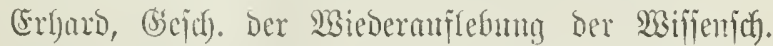
iu Ieuticulano, III, 497-ら10. 


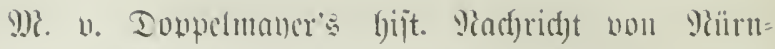
berger Minthematifern mo Simittern. 1730. p. 23 ï. Edyuazim: Notitiae le Regiomontano 1740. Boje, (5. 9) .: Nommullal de vita et scriptis 1757. Mellin, (ธ̧. Э.: Commentatio de.J. Regionontani in humanitatis studia meritis. Jenae 1763.

将anzer Amales Voll. I1. p. 233. Nürnb. 1794. Siebentees (jojrath nuto Bibfiothefar) fl. Chroutif Der Etabt 9hüruberg (ङ. 23 (5ompañmacher).

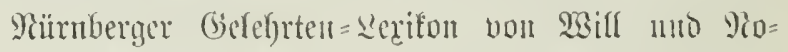
pitjc), III, 273-282 uno VII, 226--237.

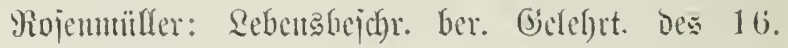
Iahrif. I. $196-219$.

Levensbeschryving ran beroemde en geleerde mannen. p. III, $59--90$.

v. Marre: Notitia trimu codicum antographorum Johannis Regiomontani in Bibliotheca Chr. Theoph. de Murr. Norimberyae 1800.

v. Miur memorabilia bibliothecarmu publ. Norimb. I. p. $74-205$ enthält cinen Briejwechjer des

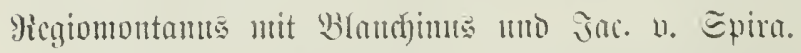

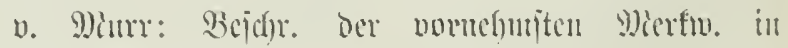
?itritb. i. ob.

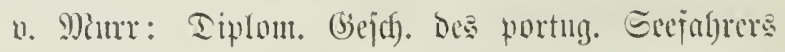
Martin Bchaim.

Baif(y), Histoire de l'Astronomic moderne I. p. 317 年. 5 3. \&aris $1775-1787$. 
Geory Shritoply Qichtenberg (geit. 1799), firoj. żll (Söttingen: vermijghte Edfriften 6. B. in Dem Reben des Sopernifus. 5. $22-28$.

Frouramm vou Dr. F. C. \&. Siffer. Sgiloburgh. 1816: Ias Qebeut Des herüfunten STtronomen umb eines

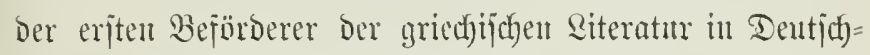

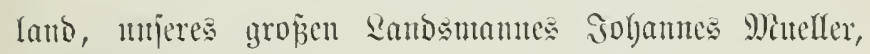
gen. Regiomontauts.

Rentrbad) ntud Regiontontamis, die Wiederbegründer einer jelbiäubigen und unmittelbaren Erjorjchung Der

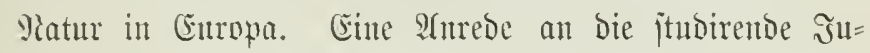
gent wou Dr. (5). 5. Edfubert. Errangen 1828 bei Parm nud Enfe.

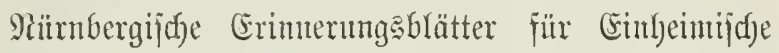
und Frembe ober 9ä̈rnbergijudje Denffääter ober Stimmen

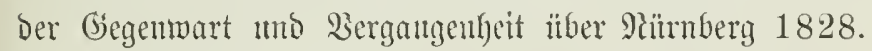
Э. 2. Edjrag's : Berl.

Die Peformation Der Sternfunde. Crin Beitray jur Deutjajen Eutturgejdjichte von Frieor. Miaufe 1852.

(Siejuidyte des Seefayrers Ritter Miartin Behaim uach Den ältejten vorbandenen Irfinnden bearbeitet von

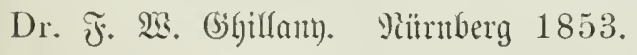

Urago, Biographie 1855 (†. Oeurres complêtes Paris 1854. 1855).

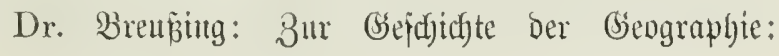




\section{$-48$}

Regiombutamm, Diartin Bebaim mo Der Tafobitab f. 3eiticf)ritt für Erofunde. Berl. B3o. IV.

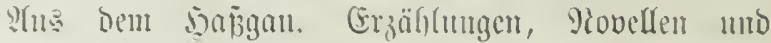

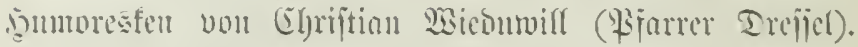

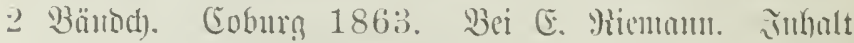
Des zrveiten Bäntochen: Biegiomontan!s.

Iic ïher Riegiontontamm handelnden Eteflen bei

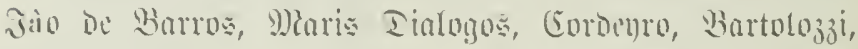
Mkafiei, Afmoretti, Wigajetta, Irigozo, Sllxamoer uou

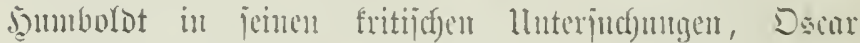

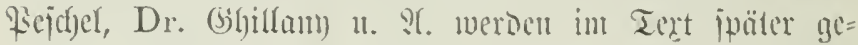
naner angejüfrt werden.

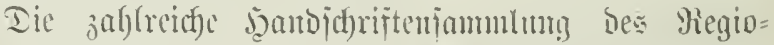

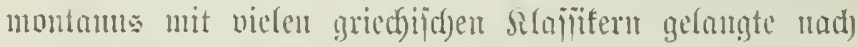

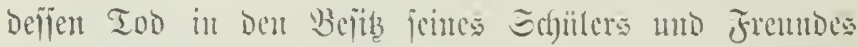

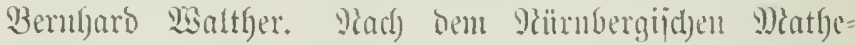
matifer Werner (i. Gassendi in Vita Regiomontani

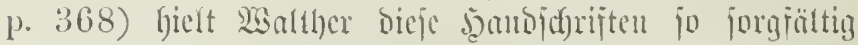

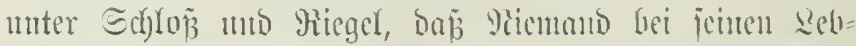
zeiten etwas babon zn jeljen nuo ju rejen befaur. Siad) Walther Tod (1504) beriuflenderten mto vergendeten

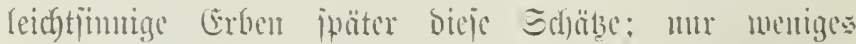

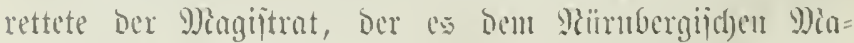

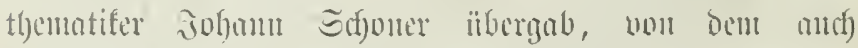

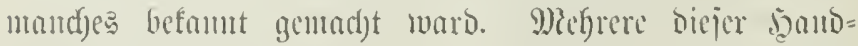

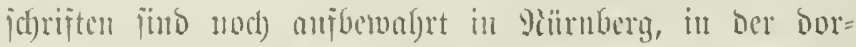

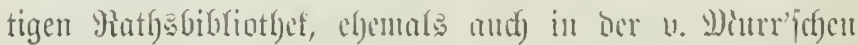




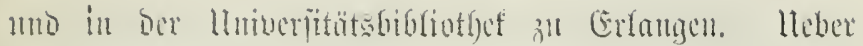

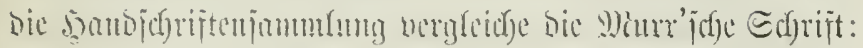
Notitia trium codicun autographorum ohamnis Regromantani in Bibliotheca Chr. Theoph. de Murr. Norimbergate 1800 . Fing (odices warm:

1) ('odex antographus Jol. Regiomontani Fol. 63 in rersionem facobi Angeli Florentini Geographiae Claudii Ptolomaci aduotationes, eine jefrer

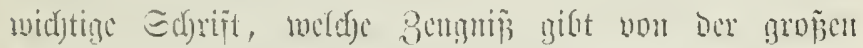

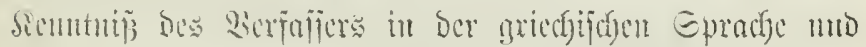

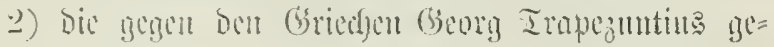

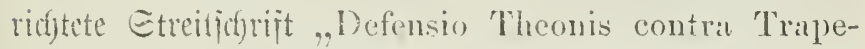

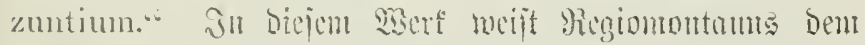

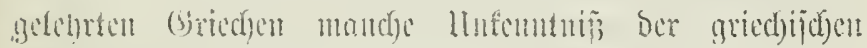

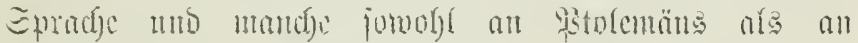

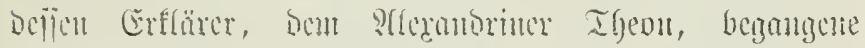

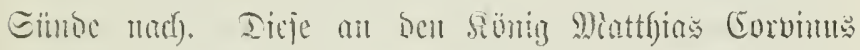

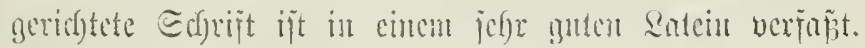

3) I) Triangulis omninodis Lib. V. Fol.

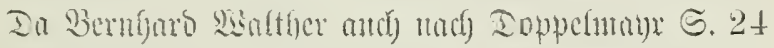

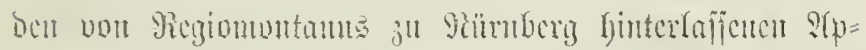

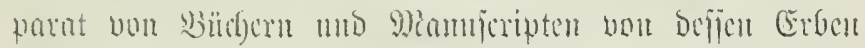

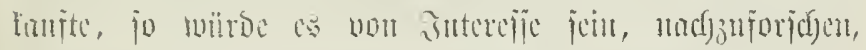

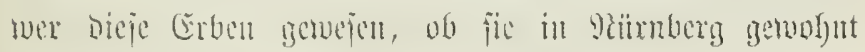
a. j. w.

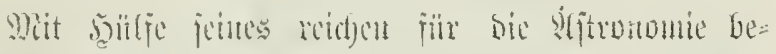

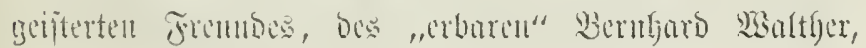




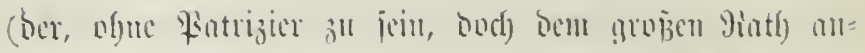

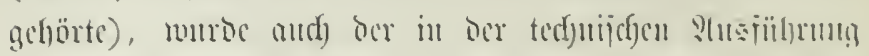

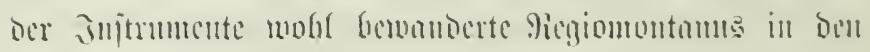

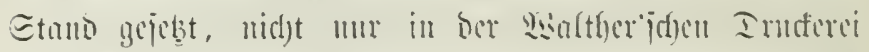

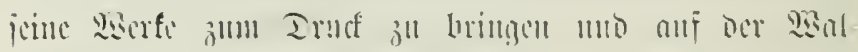

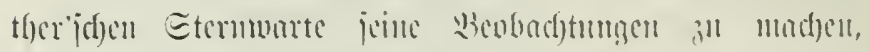

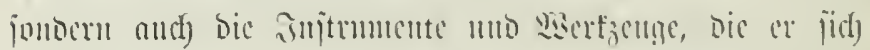

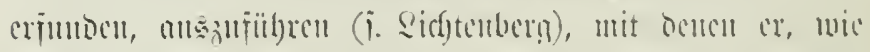

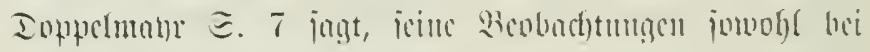

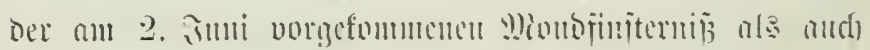

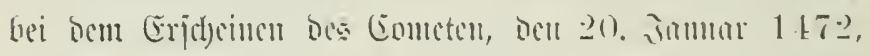
madyte. Eo murom

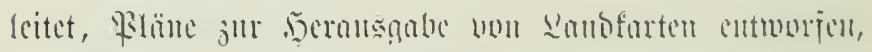

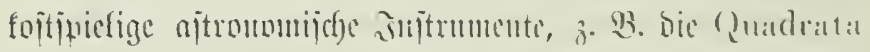
Geometrica mo Torpueta, Dic Radlii Hipparchici, dic Regulae Ptolemate Armillae mo Astrolabient mis Diciïng, Meteornscopen, Astranim, Planetarimu,

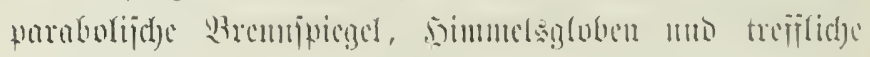

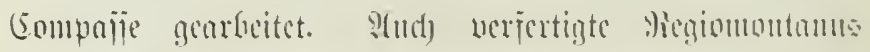
(und) Inppetmant $\Xi$. 9) in jeiner officina fabrili cin

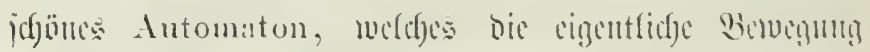

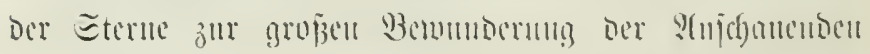
Darjeflte.

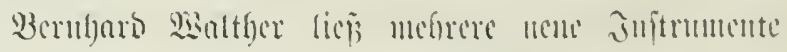

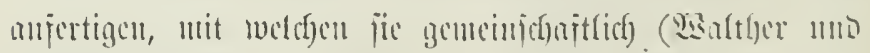

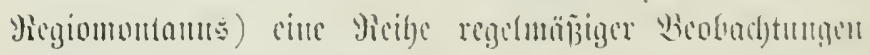

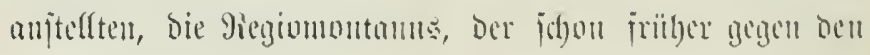




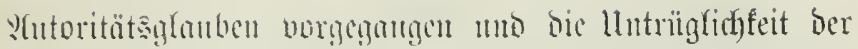

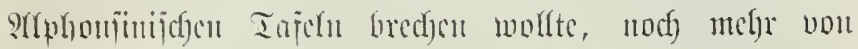

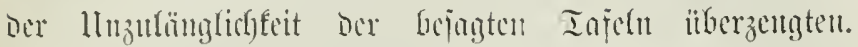

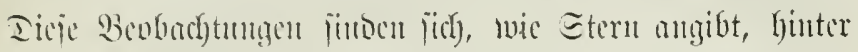

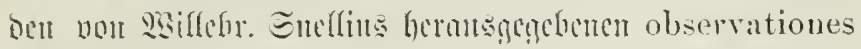
hassiarae.

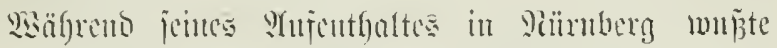

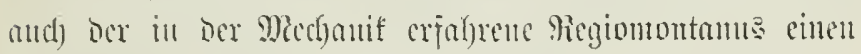

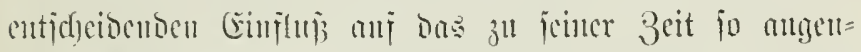

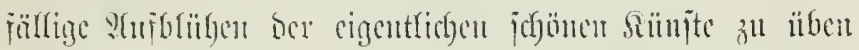

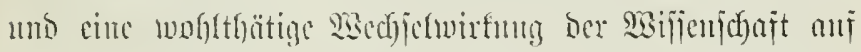

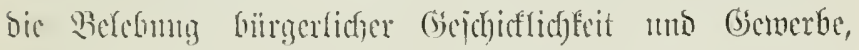

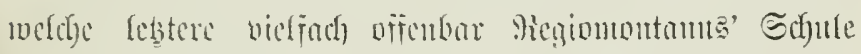

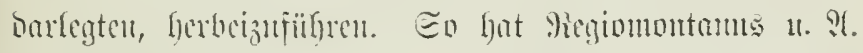

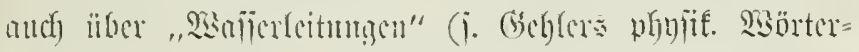

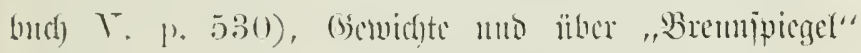

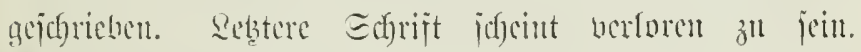

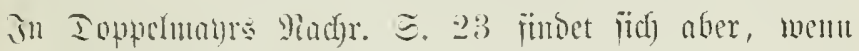

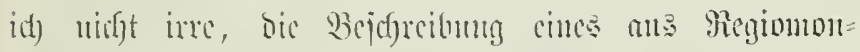

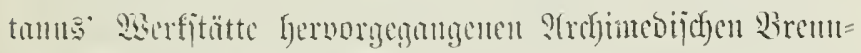
ipiegcts. Studf vout Astrolahium armillare jobrieb Megiombutame cincu Iruetat, ber in ber Siünberger

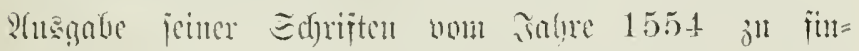
Den ijt.

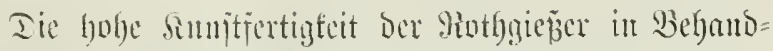
fumg bes wafridgeinfidy and in diliruberg erfuntonen

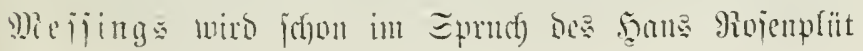


vou 1447 gepriejen mo Eiebenfes berichtet in jeines

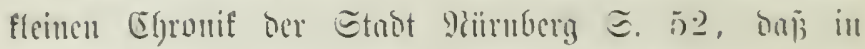

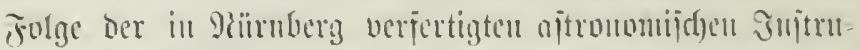

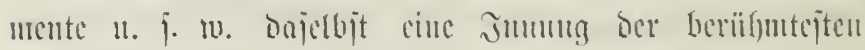

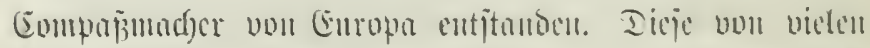

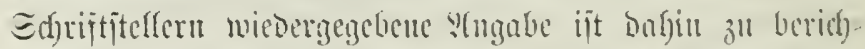

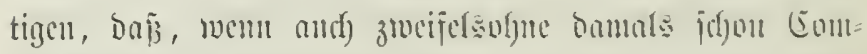

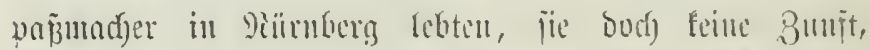

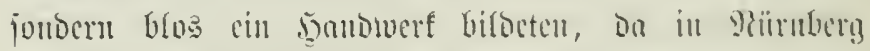

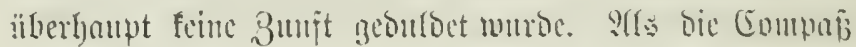
mad)er 1520 jupplicirten, ifucu cine Eroum 3 "geben,

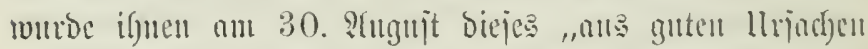

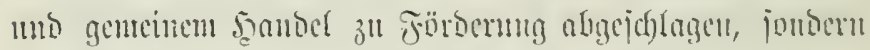

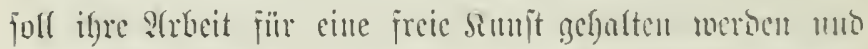

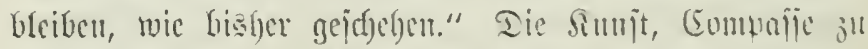

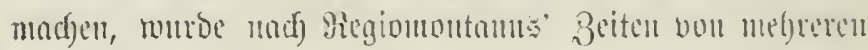

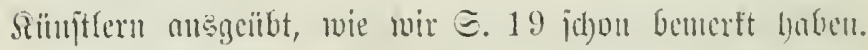

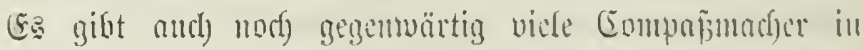

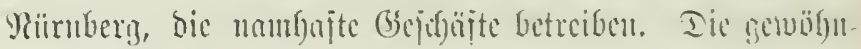

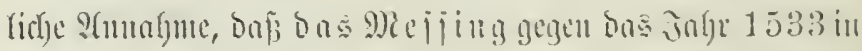

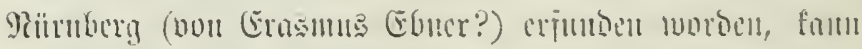

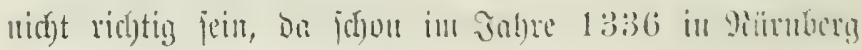

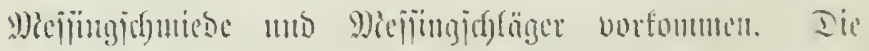

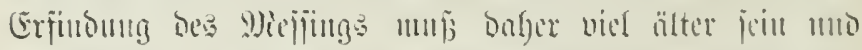

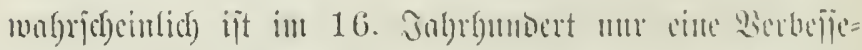

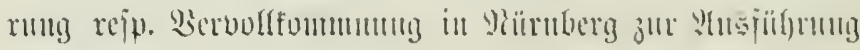
gefoument. 


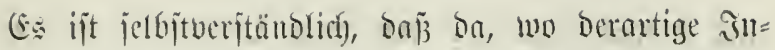

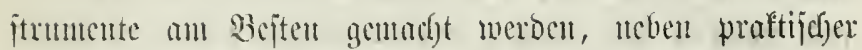

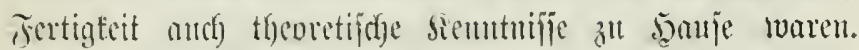

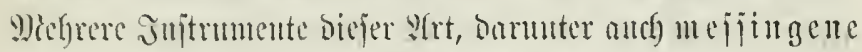
Sfitrorabien ans girgionuntam Eammlnug, die ber Minth Dor Stadt, wie bonerft, i. 3. 1505 von den

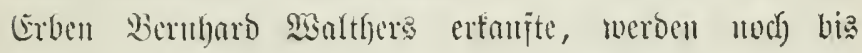

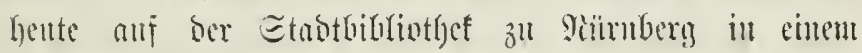

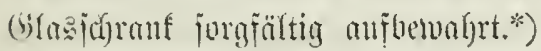

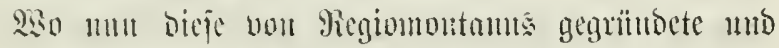

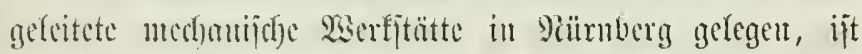
cbeu fo menig mit Bejtimuntyeit ju ermittern, als wo bos

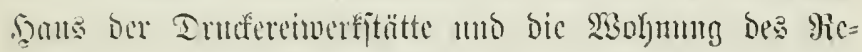

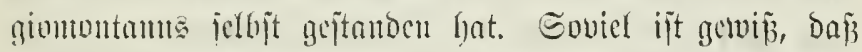

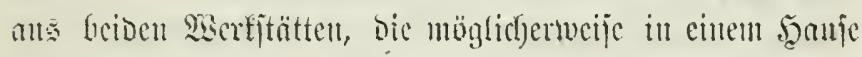
gemejan fem fümen, werthoolfe Probutete herborgegnangen

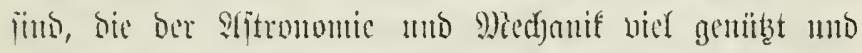

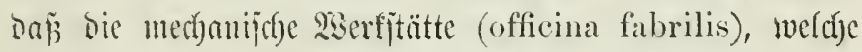

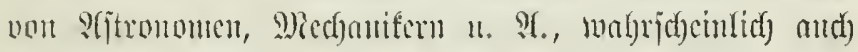
won Shartin Befaim, bejucht worden, matgematijuche umo ajtronomijibe Snjtrunente gefiejert hat, bic fïr bie nan=

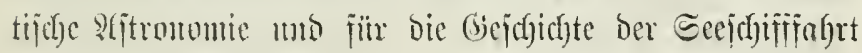

*. Istrolabium diametro 10 digitorum. 1468.

Aliud quinque digitorum diametri.

Astrolabium arabicum cupreum, diametri 6 digitorum literis cuficis, lineisque argenteis distinctum (i. Murr, memorabilia bibliothecarum publicar. Anrimbergens. p. I 9. 499 4h0 II. 317 sqqq. 
แnD Entocefungen unu cminenter Sisidftigfeit gemejen fino

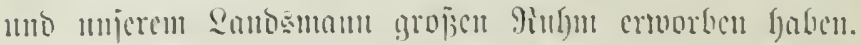

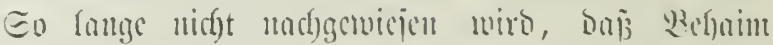

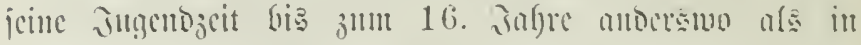

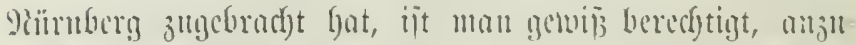

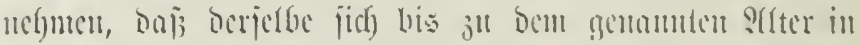

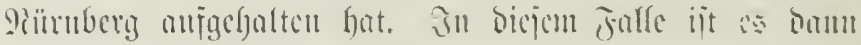

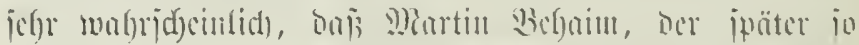

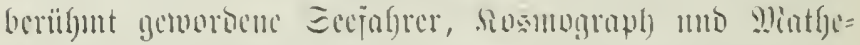

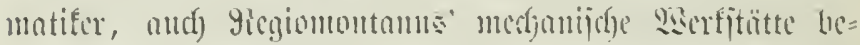
fudft hat, ba jie ifm, ocr ans cincr angejefonen Fomilic

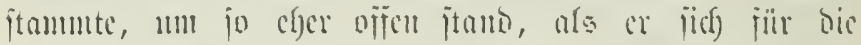

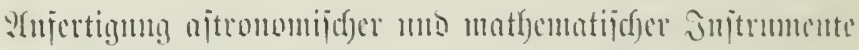

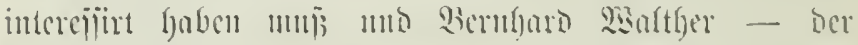

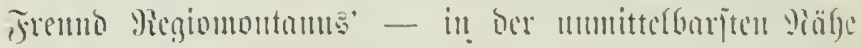

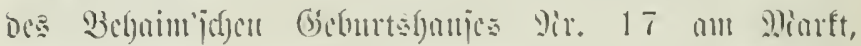

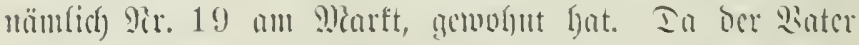
bes Eecjabrer me 1.574 gejturben ift mo ber mit

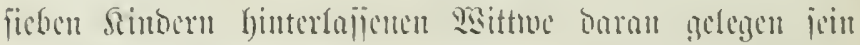

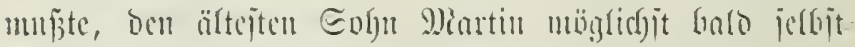
ftänbig ju machen, jo gat shartiu befaim molyt art

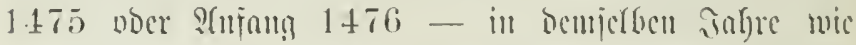

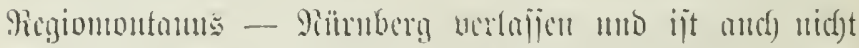

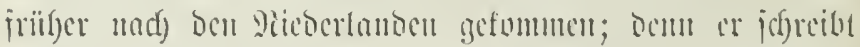

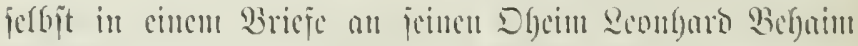

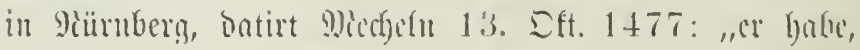

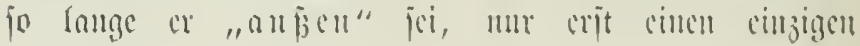




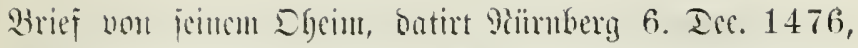

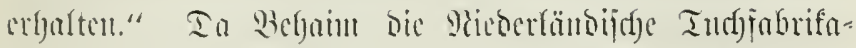
tion ans cigener ?hipt)anung femen femen jolfte, wic er

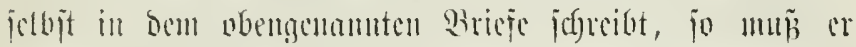

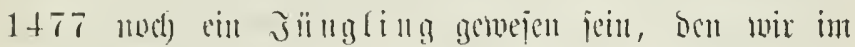

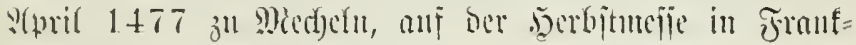

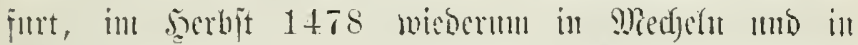

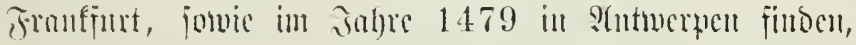

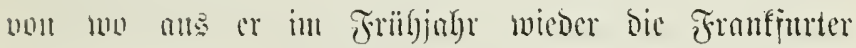

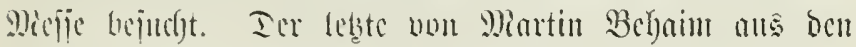

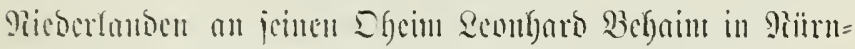
berg gericfetete Brief ift ans s(ntwerpen wom 8. Juni

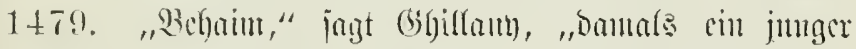

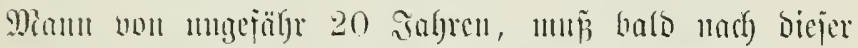

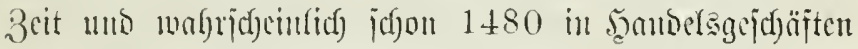

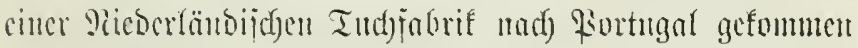

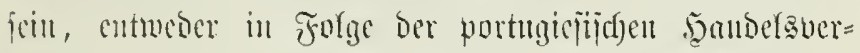

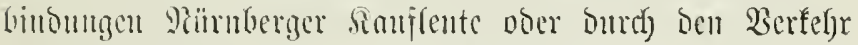

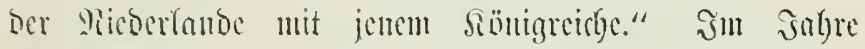
1483 ijt 3ebaint wicber bon Rortugal nad) ocm Rieder= fanben mo bon fjer nadi ?ä̈rnberg gefonmen, twie Dr.

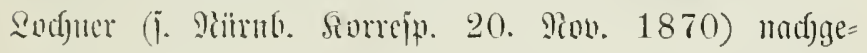
wicjen; Dem den 1. Mä̈r 1483 miro Martin Befanm ill Silimberg, weil er ant 19. Febr. befietben Jafres mit ben Jnoen anf einer Seodjocit getnuzt hatte, bei Be= fegenfycit ciner Pofizeiptraje, zu ber cr verurtfeift. Inurbe, wernoumten, Bebaim mutroe adyt Tage mb siächte uno 


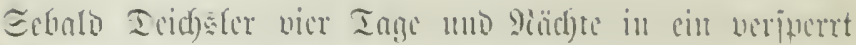

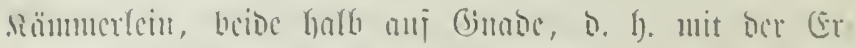

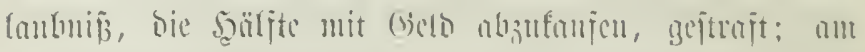

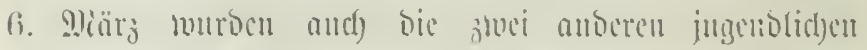

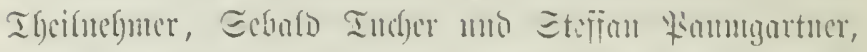

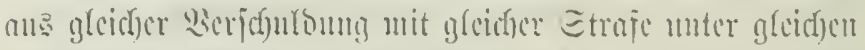

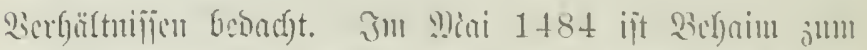

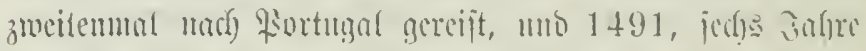

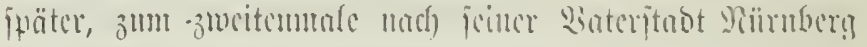
juriicfigefe(t)rt.

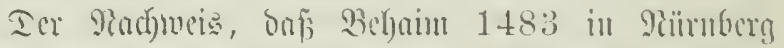

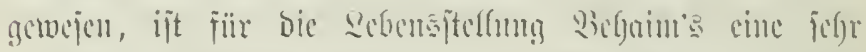

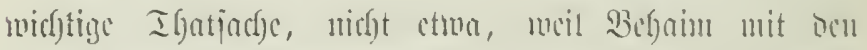

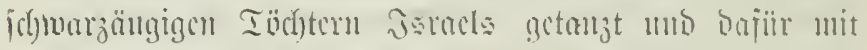

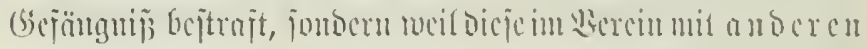

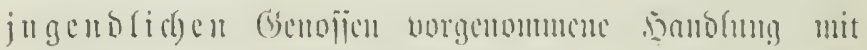

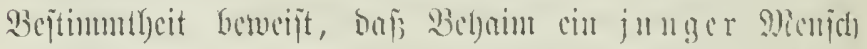

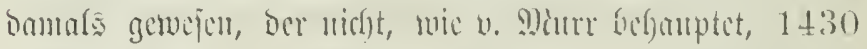

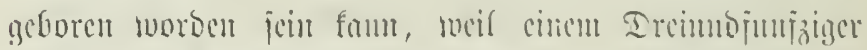

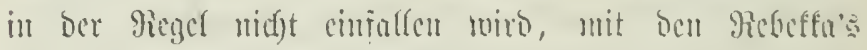
mo Eara's ber Intongajic im luiftigen Tantse fich ju brchen.

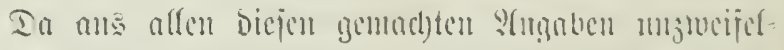

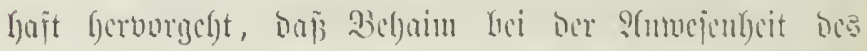

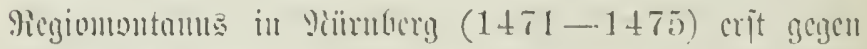

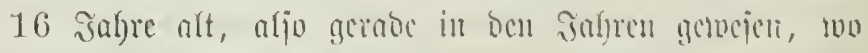
man fid hoügcren Etmbicu jumcigt, fo foum or in Fullge 


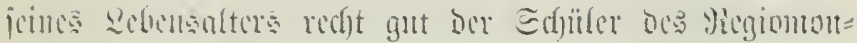

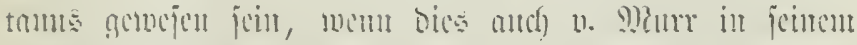

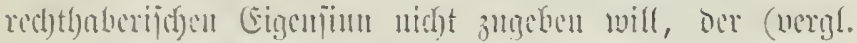

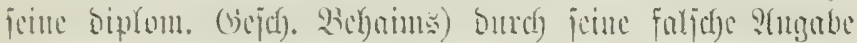

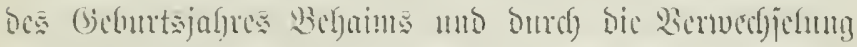

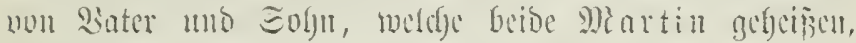

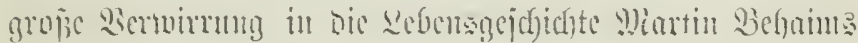

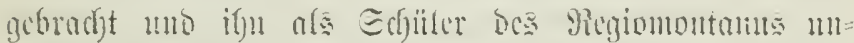

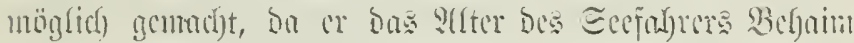

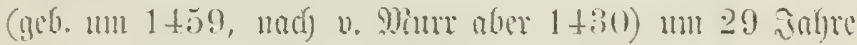
fit hoch gacict hat.

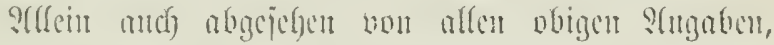

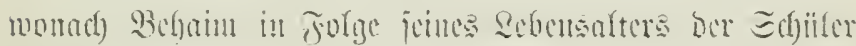

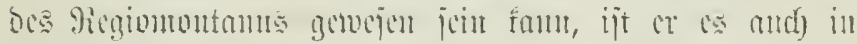

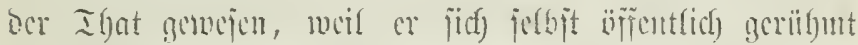

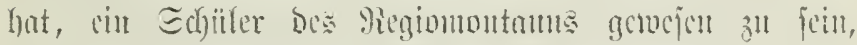

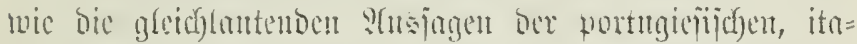

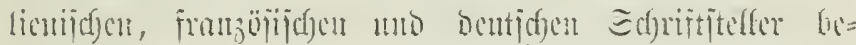

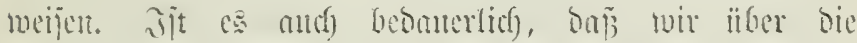

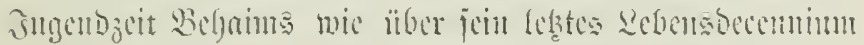

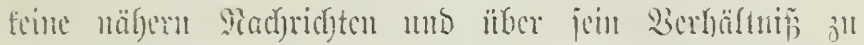

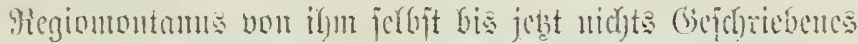
ober Geoructes bejitien mb bieffeidyt bie vben ani Grumb

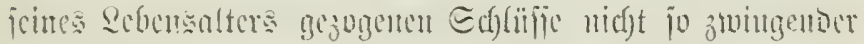

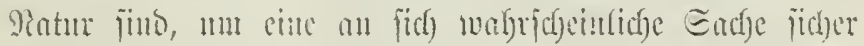

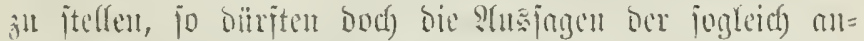

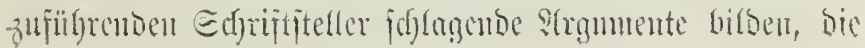




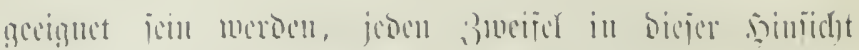

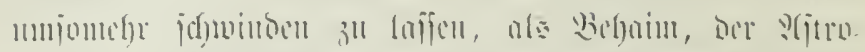

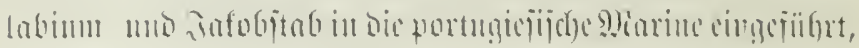
Dommas . montmus jofon in s.

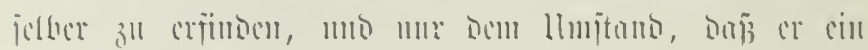

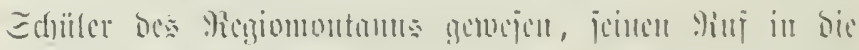

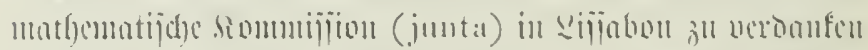

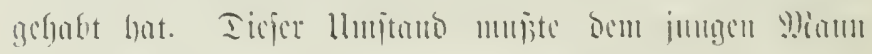

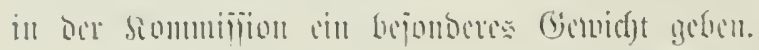

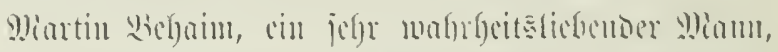

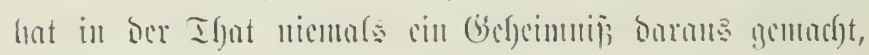

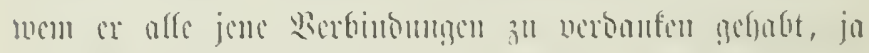

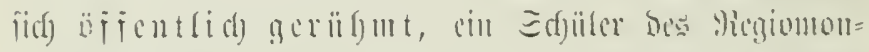

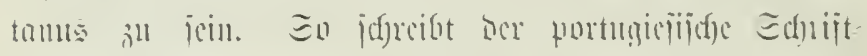
ftefer hoino de Barros in jeincu Decaden I, lih. IV.

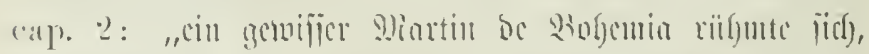

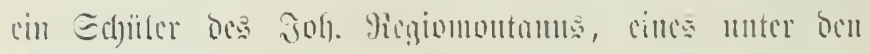

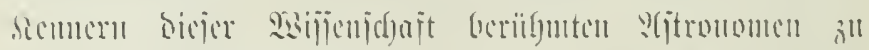

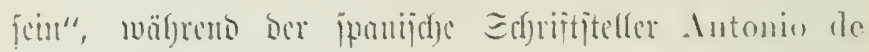

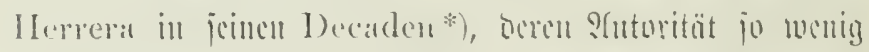

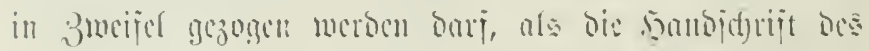

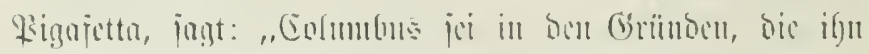

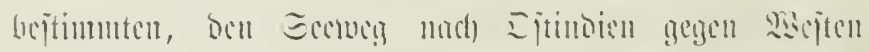

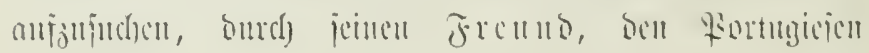

*) Herrera, dec. I, lib. I, cap. ¿े unto dec. II, lib. 2, cap. 19. 


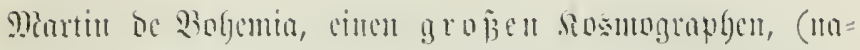

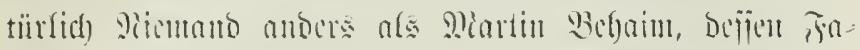

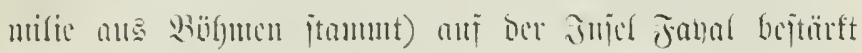

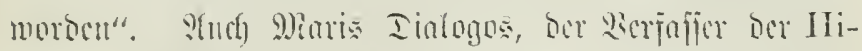

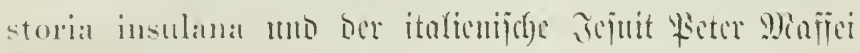

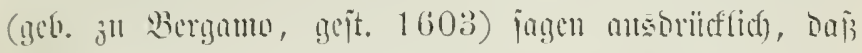

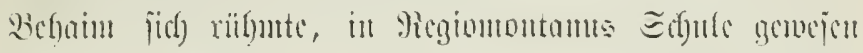

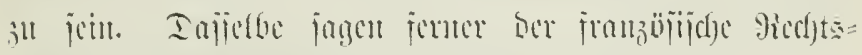

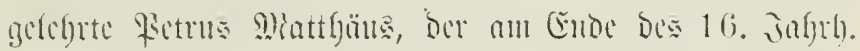

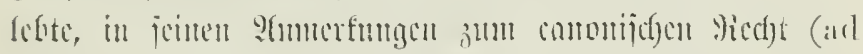
VII recretalimu lib. I, tit. IX de insulis novi orbis),

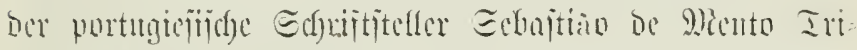
gुoza (Memorials de litteratura Portugne\%al ete.) uno

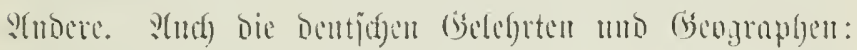

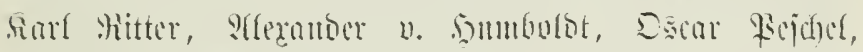

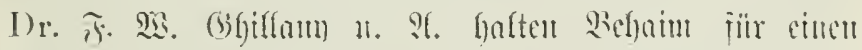

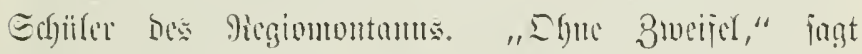

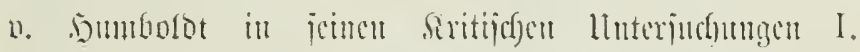

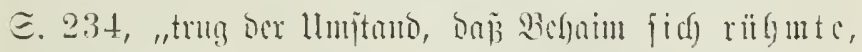

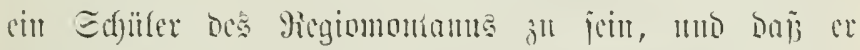

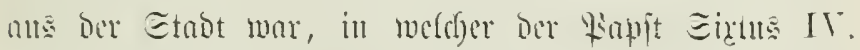

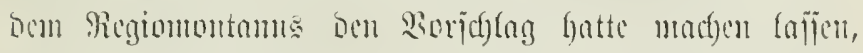

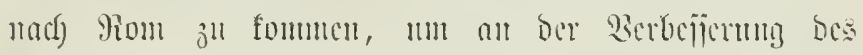
Salender zu arbeiten, wejentfich Dazu hei, feinen giuf

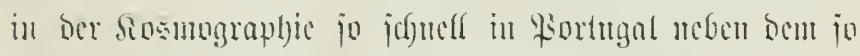
vieler anderen Miänmer zu begrünben, wefdye fich mit

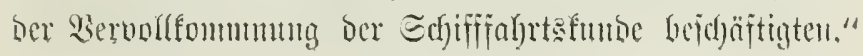




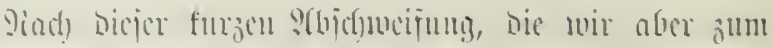

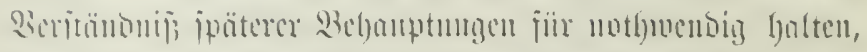

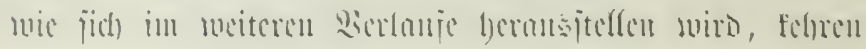

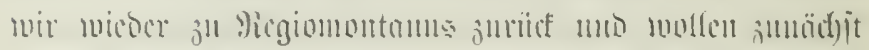

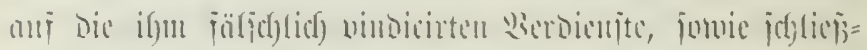

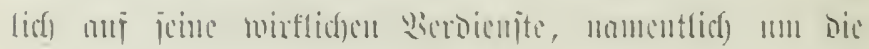

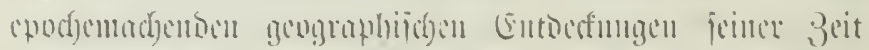
nälyer cingelyen.

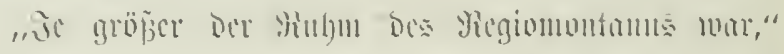

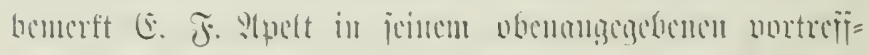

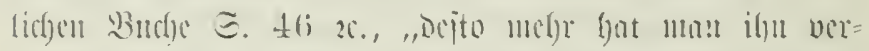

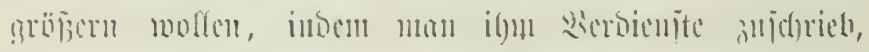

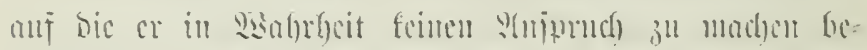

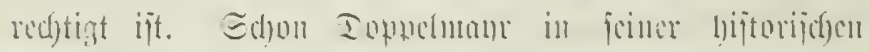

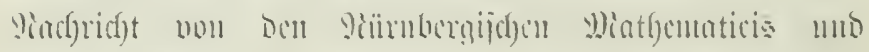

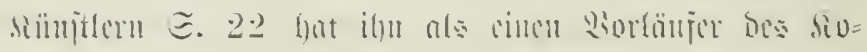

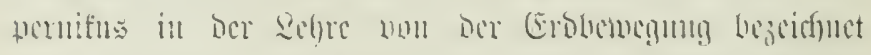

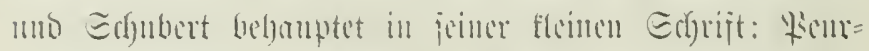

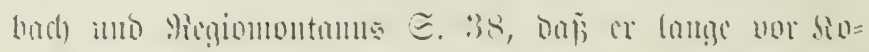

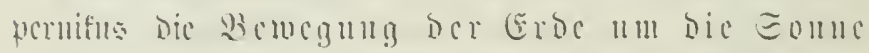

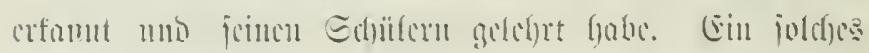

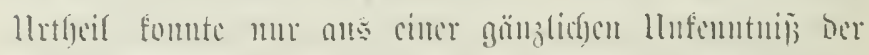

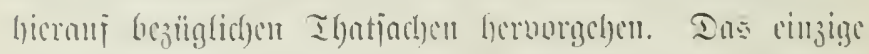

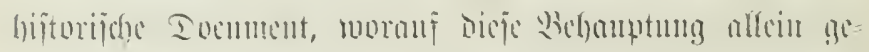

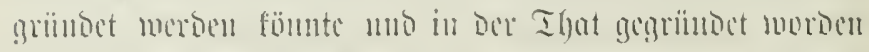

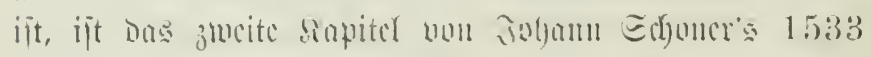

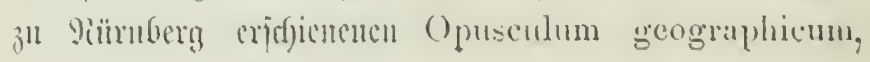




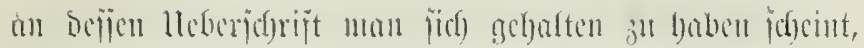

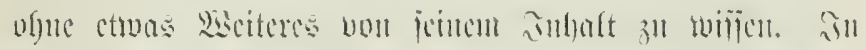

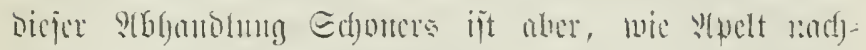

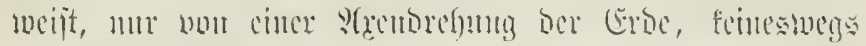

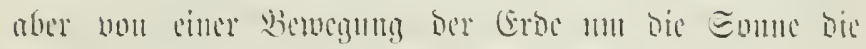

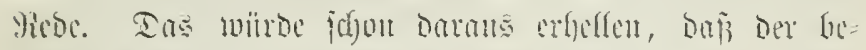

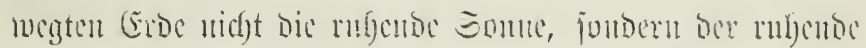

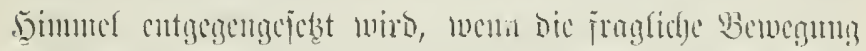

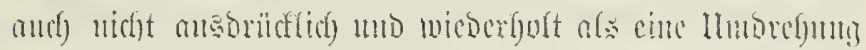

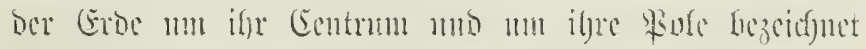

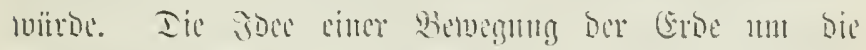

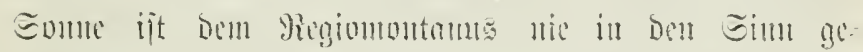

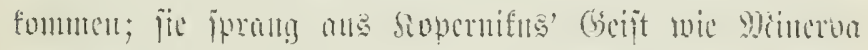

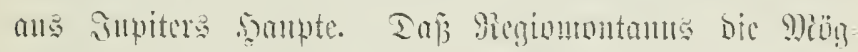

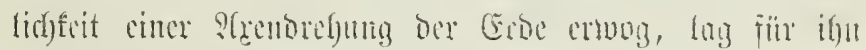

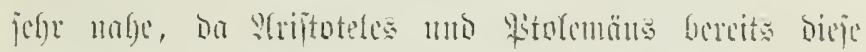

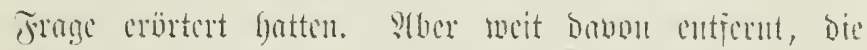

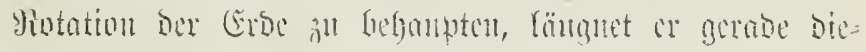

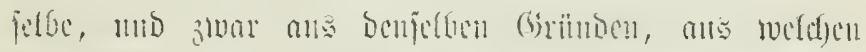

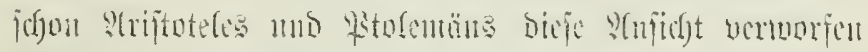

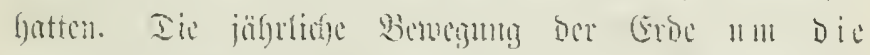

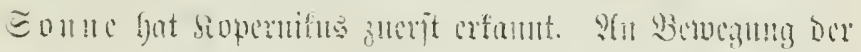

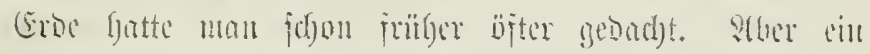

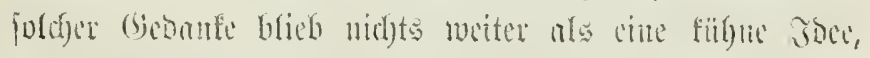

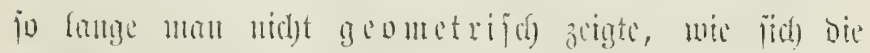

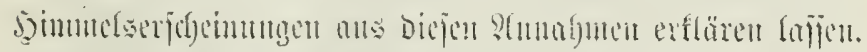
Das max ca, was sioperuifus leijtete." 


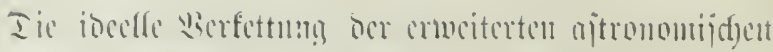

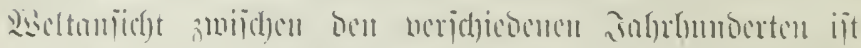

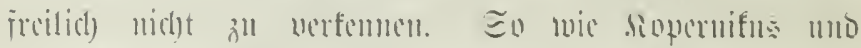

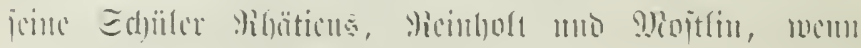

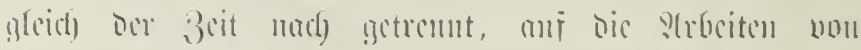

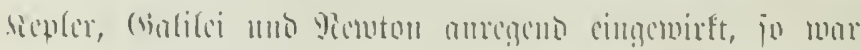

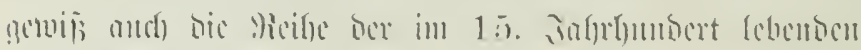

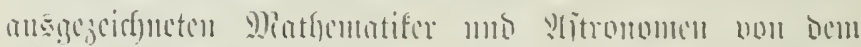

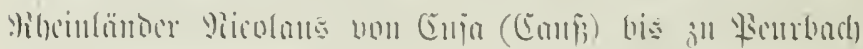

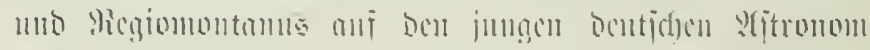

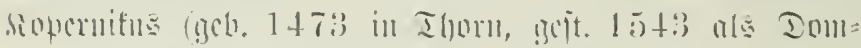

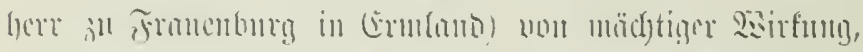

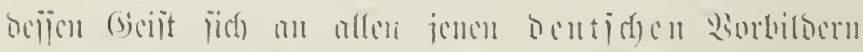

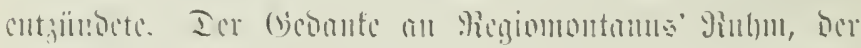

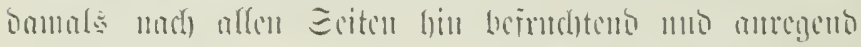

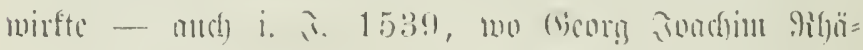

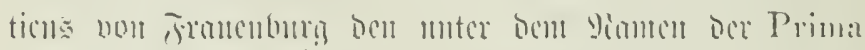

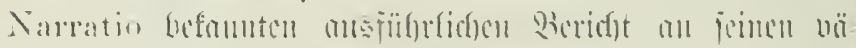

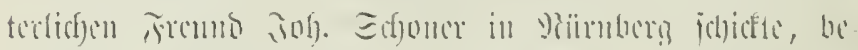

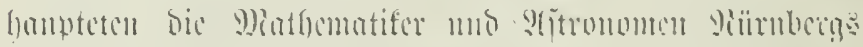

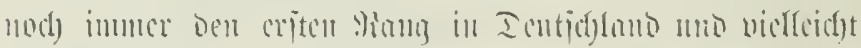

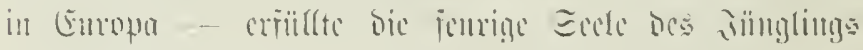

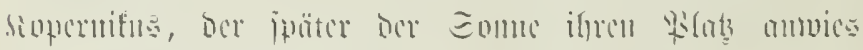

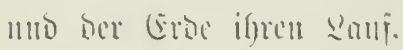

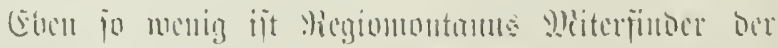

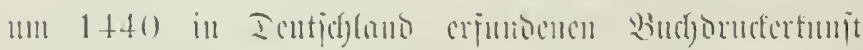

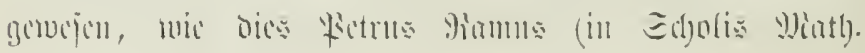




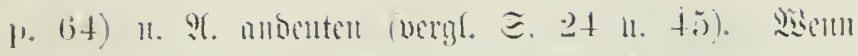

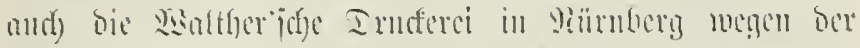

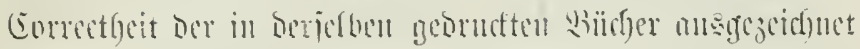

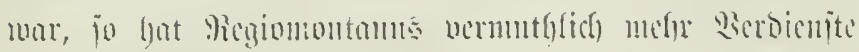

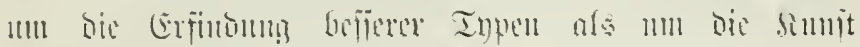

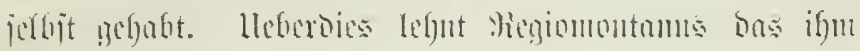

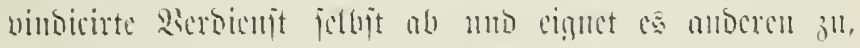

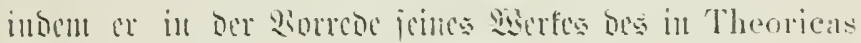

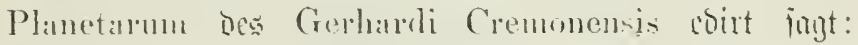
Quis nescit mirifican illam formandi artem muper ¿ mostratibus excogitatam?"

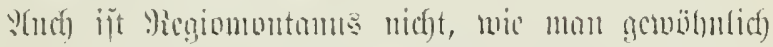

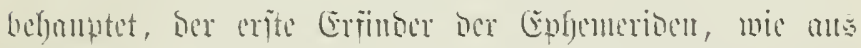

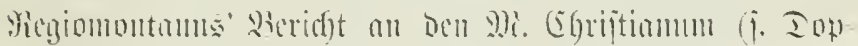

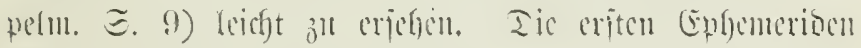

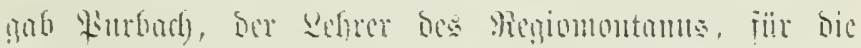

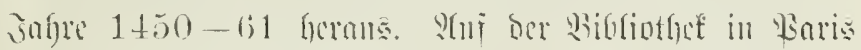

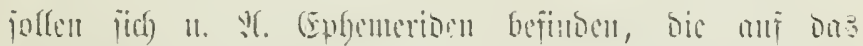

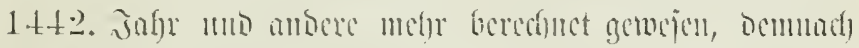

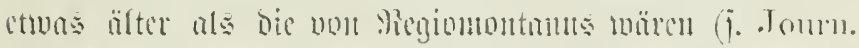
des siar 1702 1). $3+7$ mo 年. Gifan in vita Re-

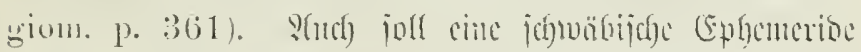

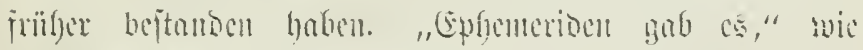

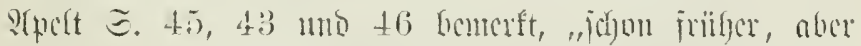

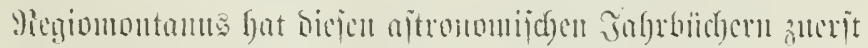

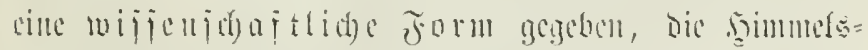

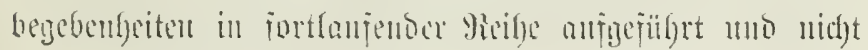




\section{6.}

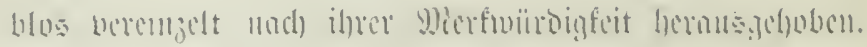

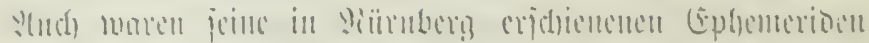

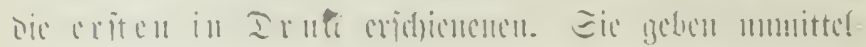

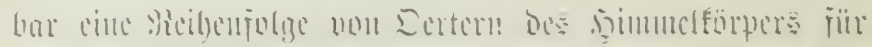

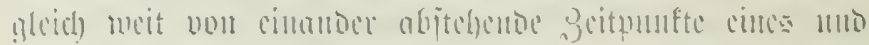

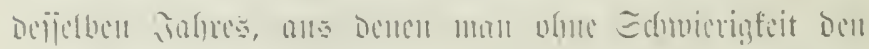

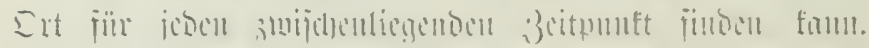

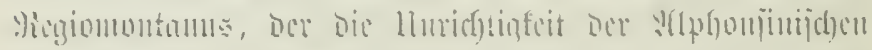

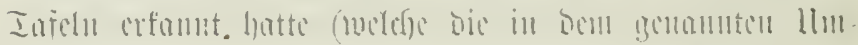

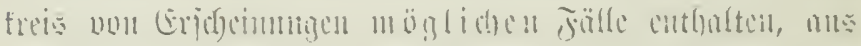

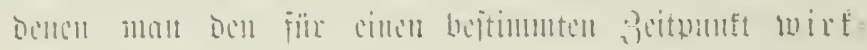

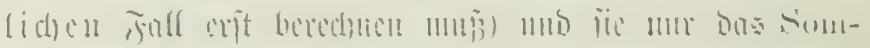

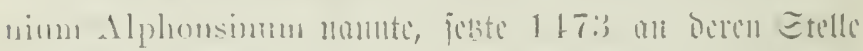
jeine Ecritgunten n

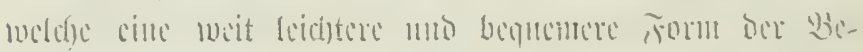

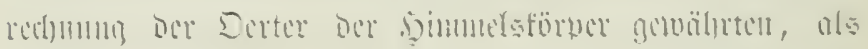
dic Intilli."

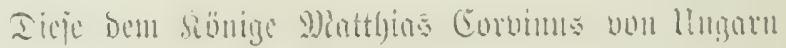

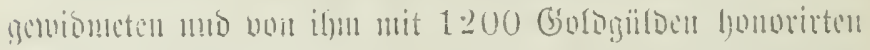

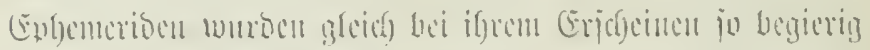

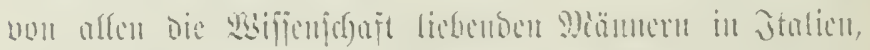

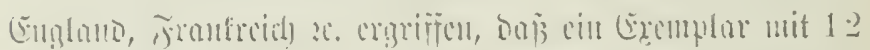

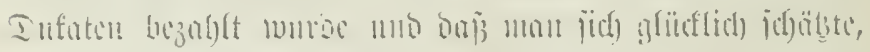

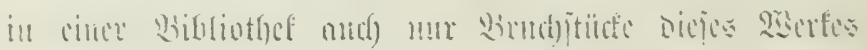

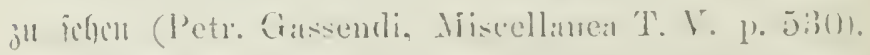

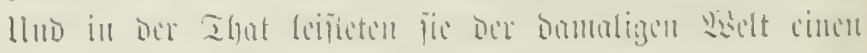

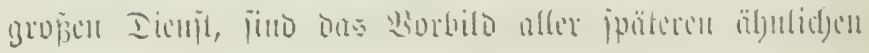




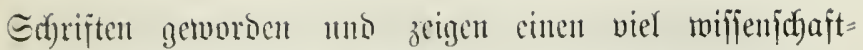

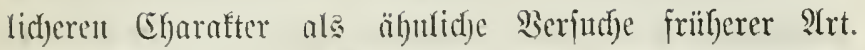

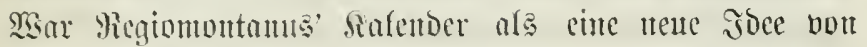

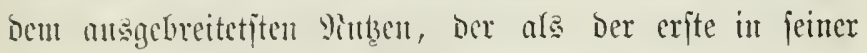

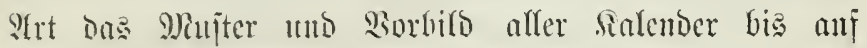
Die nencite 3eit gewordon, jo befanten bie Ephemeriben

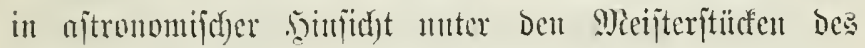
menichlict)en

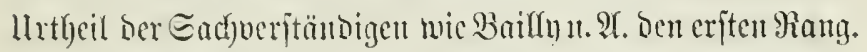

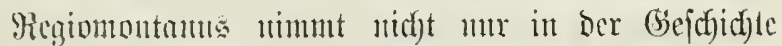
ber sitronomic, jonbern viefmelys nod) in ber (5ultur=

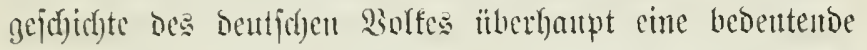

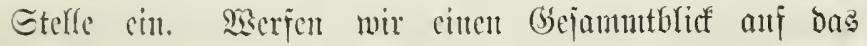

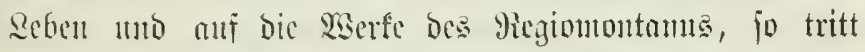

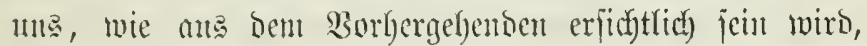
biejer bedutiane beutiche Mann in vier djarafterijtijchen

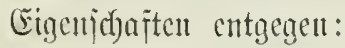

1) als ein groper Bejürocer ber griechifden Spradie mo siteratur in Dentiditand vergl. ธ. $6,7,8,9$,

2) als cin groper Beförocer ber Mathematif, Der ?frgebra mo Trigonometrie \{. $5.7,16,36,63,64$,

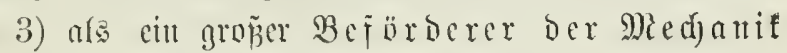
i. ธ. $50,51,52,53$,

4) als ciner ber bedentenditen dentjden

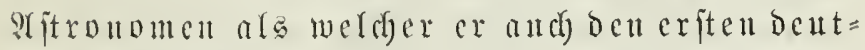
jucu Sartuder gejdrieben fat, f. S. 28, 29, 30, 31. 


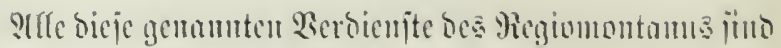

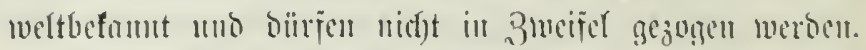

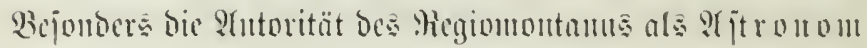
faum in feiter saceije bejueijelt mo bejtritem merbeu;

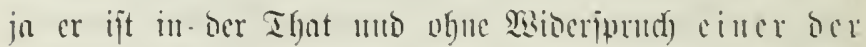

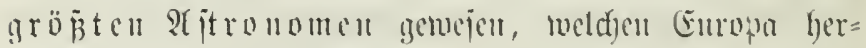

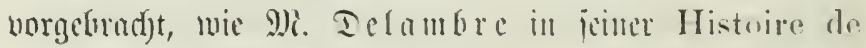
l'astronomie du Moyen àge. Paris 1819 p. 565 ("Regiomontan itait sims contreclit le plus savant astronome qu' ent encore produit Earopers) jefle ridftiç

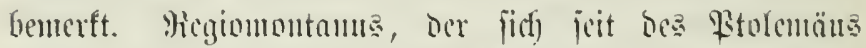

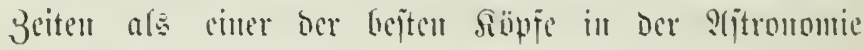
hervorgethan, ijt nidgt mur nfa oer Bater ber neueren

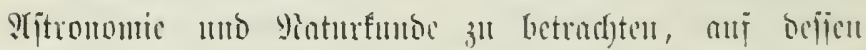

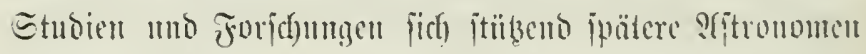

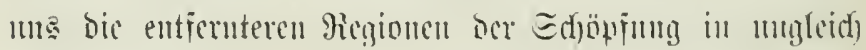

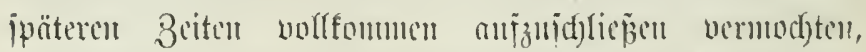

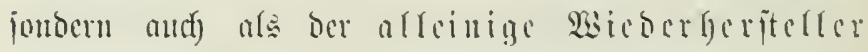

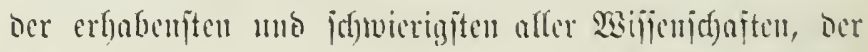

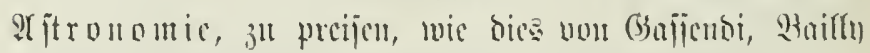

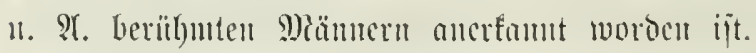

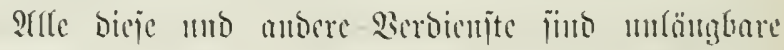

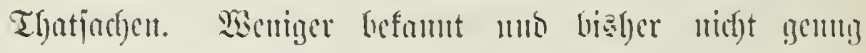

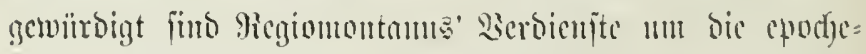

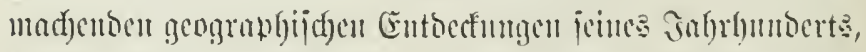

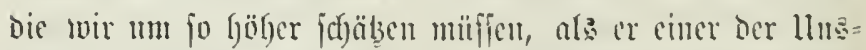
rigon gemejen, oer tiej in bentichen Bintentande geboren 


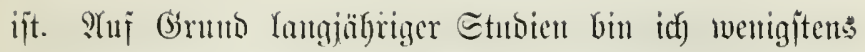
zll ber feîtell lleberäengutig gefangt, baj Regiomontanus

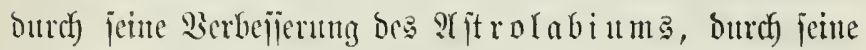

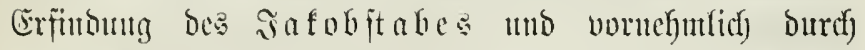

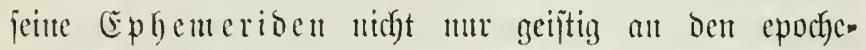
machenoen Estutbenfungen jeines Jafyrhunoerts betheiligt

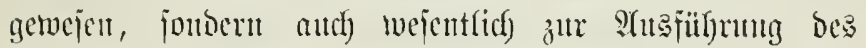
RIanes vou Columbus, nach siejten zu fegeln, beige= tragen bat - mitfitu itl eriter Sinte ebenfo rote

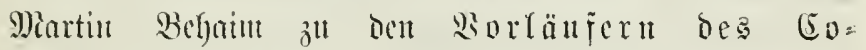

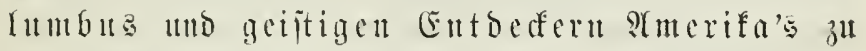
उäh)len ijt.

Meinen obigen Bemerfuttgen, onż Martin Behaint

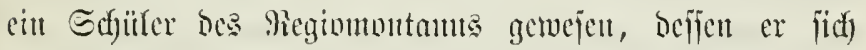

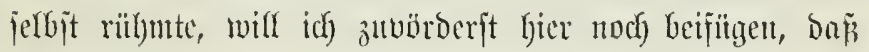
bic bcioen Ecejafrer Bchaim un Columbus, wic aud

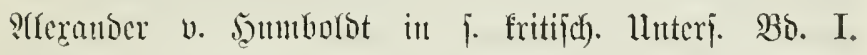

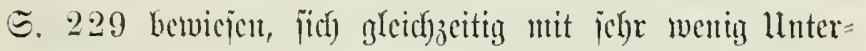
frechungen in ben Jabren 1482 und 1484 it Siffiabon

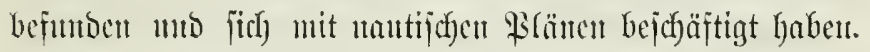

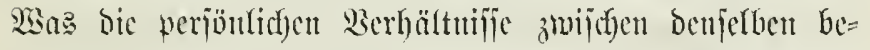

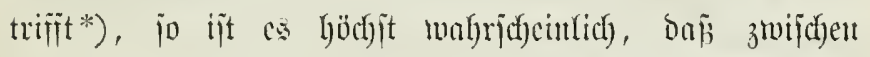

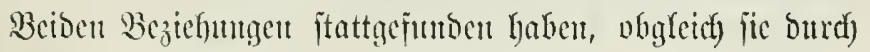

*) Columbus, 1456 geboren, foll fajon 1470 in eincm alter bon 14 Эabren juerft nad) Sifjabon gefommen jein vergr. Auslano Sir. 50, 11. Dec. 1866: lleber das Geburtsiagr des Columbus. Zou Dr, Desc. Bejdel. 
nid)ts mmittelbar nad)gemicjen werben fömen, Imen aud), wie jodjon oben bemerft, beerrera jagt, baj (5olumbus in jeinen Jocen $\ddot{b} b e r$ bic geringe Entjernung ber Ditfiijte von :(jia Durd) jeinen Freund 9)intin oc

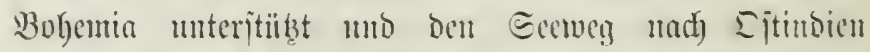
gegen Sisejten aujzujuchen bejtürft worbeu.

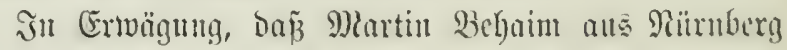
Der Edjüler bes giegiomontant: mo mit Columbus be=

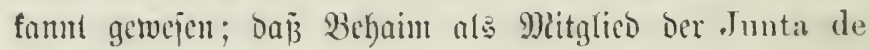
Mathematicos angefourt, weldye nad) Barros Dec. 1.

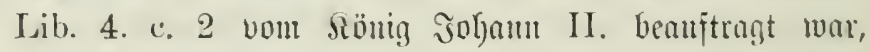
eine Methode anzugeben, nad) ber ভoumenhöhe zu idjiffer (maneira de navegar per altura do Sul);

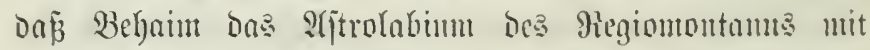
Der jtereograplijhben Dorizontalprojection gefannt, joldes wahridfeinfich bei ber Jnuta vorgezeigt unb fief)er auf jeinen jpäteren Reijen mit Diego Cano ober Càn ge=

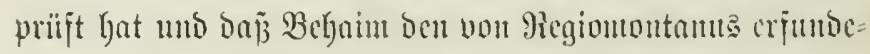

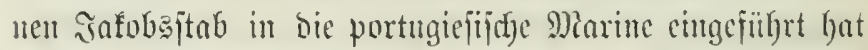

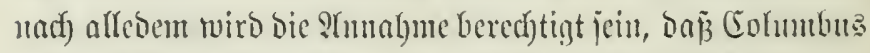

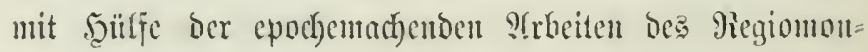
tanus, bejen Ephemeriben er itberbies nachers= (ic) mit am Bord feilles Schiffes batte, mo

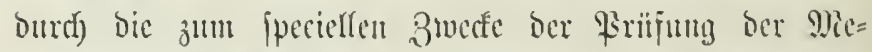
thoben unternounuene geije Martin Bebaims feine groje

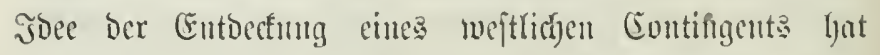

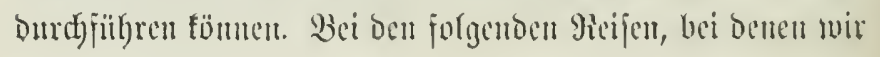




\section{$-69$}

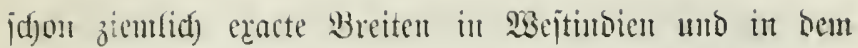
amerifanijafgen Continent niedergelegt fütben, fam ber Ëmflñ biejer Strbeiten fanm bezueif̣elt werden. Die in Den Jalyrell 1500 mis 1508 erjofientenen Sarten

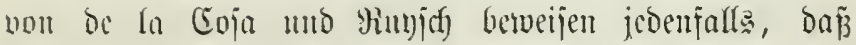

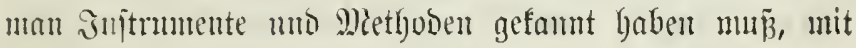

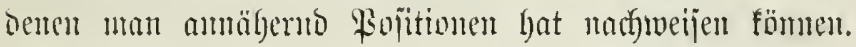

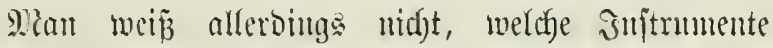
Columbur anf jeiner Reije bemtze lyat; ba aber bou italienijgen Jujtuntenten jedenfalla feine Rede ijt, jo mitifen es beutiche apparate mo Mietgoden gewejen

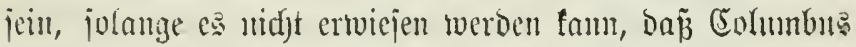
italieniigac bemubzt lyat.

Ia Columbus, der bie erjten \&ängen jür zmei

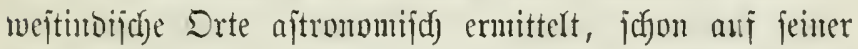
critten Bicife cine bejtimmte Breite angibt und nads.

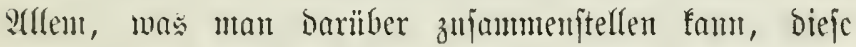

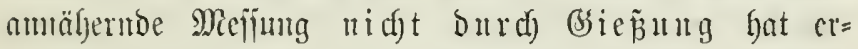
lantgen fömen, fo mun er ntothwendigerweije fid) eites Inftrumentes zur beftimung und Beobachtung ber Eonnenlyöge bebient haben, un fidd in ber Breite jull orientiren, ba Gefountlidf) bur(t) Den Contpa man wohll nach der șinmelagegend ftentern faum, der Eeefahrer aber ungewiés bleibt, in welden Breitengraden ber Erofungel er fich befintoet.

Und weldjes Inftrument wäre zu biejent Bwecfe

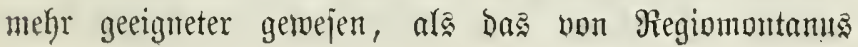


nerfertigte verbefferte ? Iftrofabium uno ber nou ifmerju=

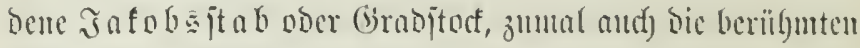

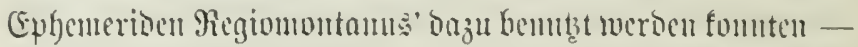
jene njtronomijiden Iajelu, in benen ber Ert bes Etmoes

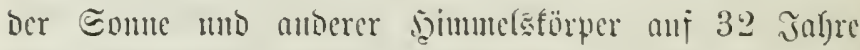

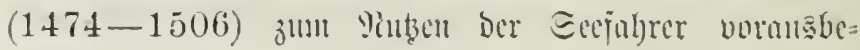
rectinet war?

Sit and bas angeblid) vou seippard)u $(16+-128$

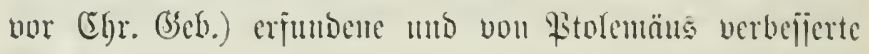
S(jtrolabitum - \$tolemäus jpricht im britten sapitel bes arften Budfes jeinter beographic wou cutem injtru=

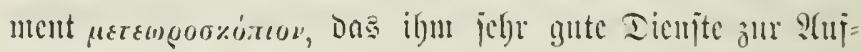
finturg ber \&änge แแ⿰ Breite our Drte geleiftet babe

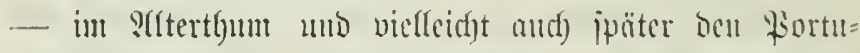
giejen befamm gewejen, fo ijt bod) jein (Gidrandf), wie

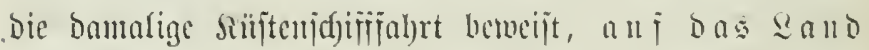

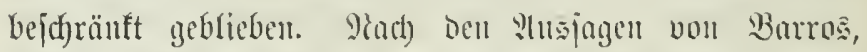

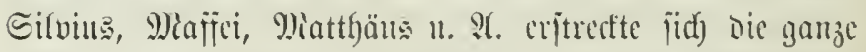
bamalige Edjitifafhrt ber Eevleute mur läntys oer siijte.

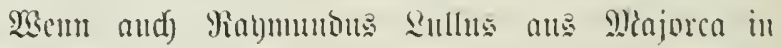
jeiter Arte de naregar jwei Jahrlfumberte vor Beljaim

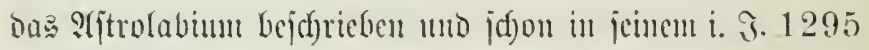
nerfaf̧ten Budfe: „Fenix de las mararillats del orbe6.

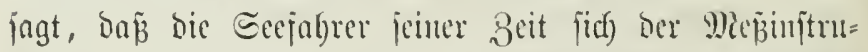
mente, Der Eecfarten mis ber Miagnetundel bebienten (tenian los mareantes instrumento cartil compas !

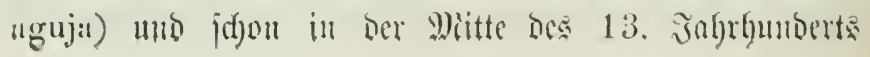


in Der Marute Der Catalauer umb ber Snjel Majorea nautijuc suftrumente üblid waren, um bie Beit burd) Eternfg̈hen ju finden unt twern anth bie

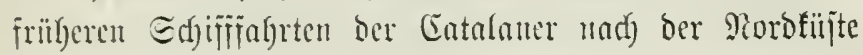

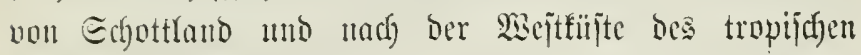

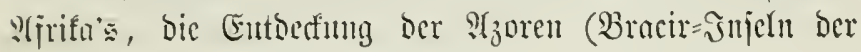
Meltfarte von Picigann 1367) burdy bie Pormönner $\mathfrak{u}$ j. W. une crinucrn, baj Iange vor Cohumbus man ben weit=

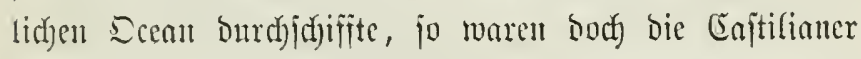

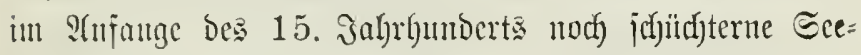
jabrer, bie fid jefbit in Mittelmeer ängittich an ben

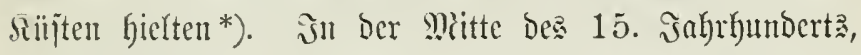
un biejelbe Zeit, wo Der Portugieje Plonius ben von Regionuntanus erjubenen Gradjtodf zum Beobaditen aui Der Ser bejdreibt (Coimbra 1546), erjajen in Epanien bas jeiner Zeit berilfmte $\mathfrak{S e r f}$ von Mebina ïber Stener= mants:

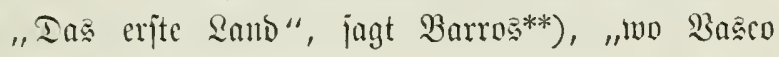
be Gama vor jeiner Sfufunjt am 23orgebirge ber guten Şofïnutg anlangte, war bic Bai, bie wir jeb̧t Et. Şelenta nemen, fïmf Mronate, nadjben cr von Sijiabon abgejegelt. Şicr jtieg or an bas Sand, un :

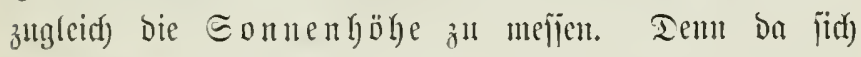

*) (5ilid. Des Zeitafters ber Entbectungen. Sion D. Befder. ธ. 160 .

*) Barros Asia Dec. I. Lib. IV. c. 2. nach Dr. Ereufing's

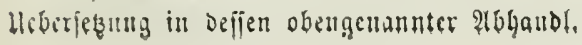


Sic Eeetente Diejes gieidje erit jeit furjer Beit ju biefen Gefdjäpte bes ?fitrolabium bebienten und bie Edjiffe flein marelt, fo getraute or fird) wegen bes

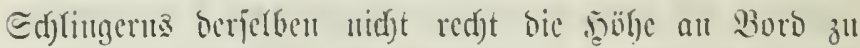

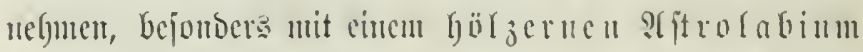
non brei Rafunen Iurdymofier, bas man auj cinem Irei=

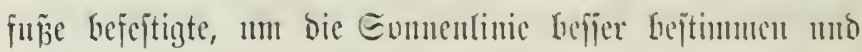
bie wahre Däbe jencs Drtes genanter mo richtiger antghen zll fömen, obroble man and fletuere sfitrolabien

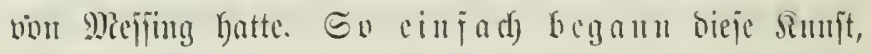

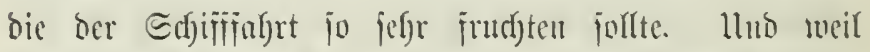
biejerbe in biejen Piedace juerjt auj bie Edjifjalurt angewendet wurbe, jo wirb es nidft unpajiend erjejeinen,

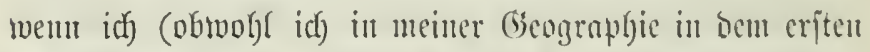

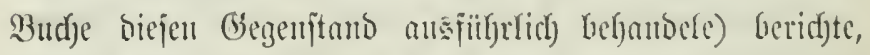

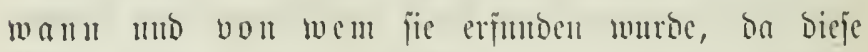

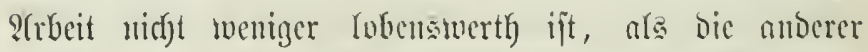

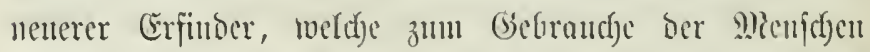
bienlicfe Eadyen bergejtellt gnben. 3m 3cit, abs ber

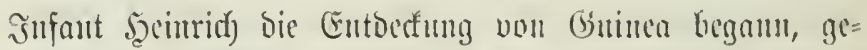

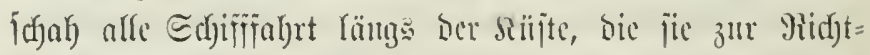

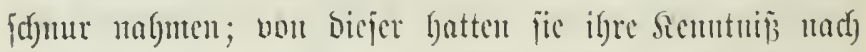
Beidjen, ans benen fie "Eegelanmbijungen" madjten, wic

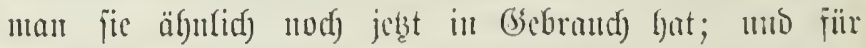

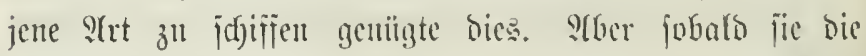
entbeften Reidye jo befalyren wolften, baj jic bie Siijte

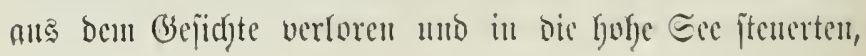


erfanuten jic, mie jefre jie jid) in ber E(f)äbunt uns

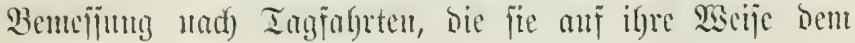

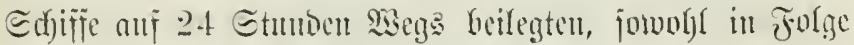

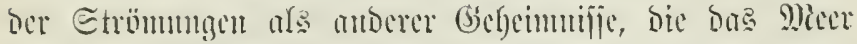

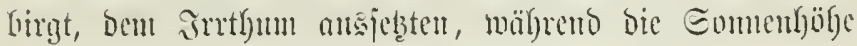

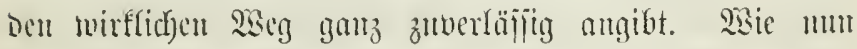

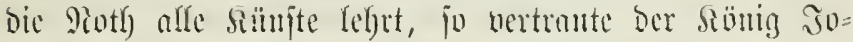
yom II. biejes Gejejäft in peiner Beit bem Micifter gio=

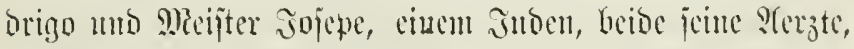

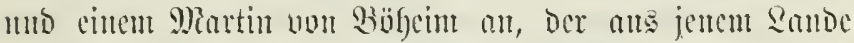

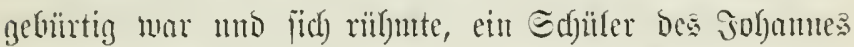
Regiontontants ju jein, cines nuter ben fientem bicjes sijijenjefaft berïfmten s(jtronumen. Dieje erfandon num

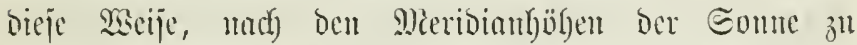

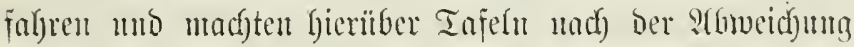
berferten, wic es jekt unter den Eectenten im Brantde ift uno ztwar genauter ale jul ?tufang, wo man fich nod) biejer grofaen bülgernen afitrolabien bediente."

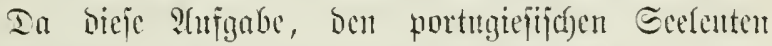

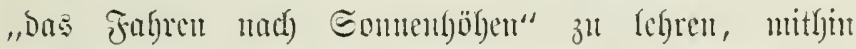
Mittel an bic jęand zlt geben, wie man bie Breite und

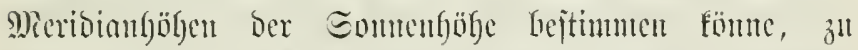

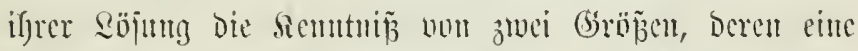
bie 9) Der Some ijt, nerfangt; die S(lphonfinijd)en Tajeln von Denen Des giegiontontamb gaitz abgejegen - ben Drt ber Somte bamals aber fojom fo gemm angaben, 


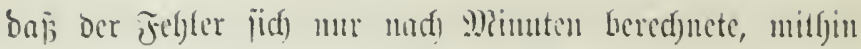

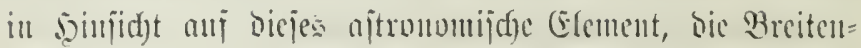

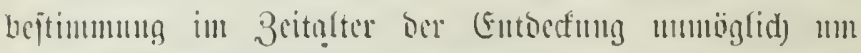

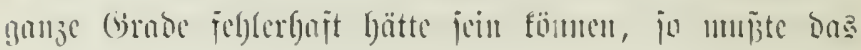

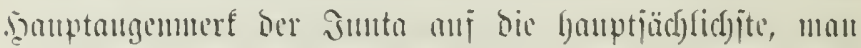
möd)te jagen, bie cinzige Esf)mierigfeit geridftet jein, bie

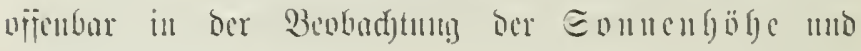

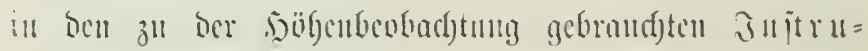
mentenlag.

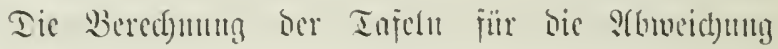

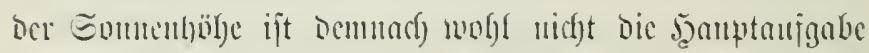
ber Junta gewejen, fontorn bie sul ber Beobacktung ber

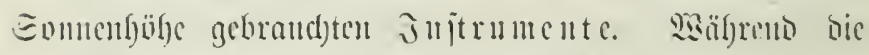

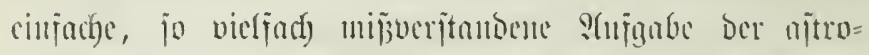

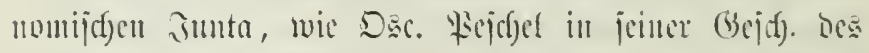

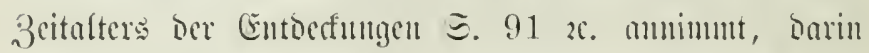

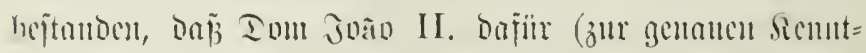

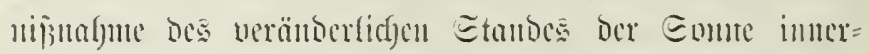
balf ber Jafreszeiten ant Taye ber Brobact)tung) neue

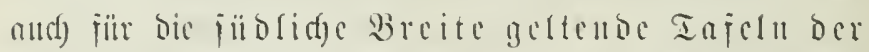

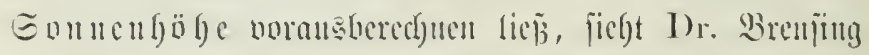

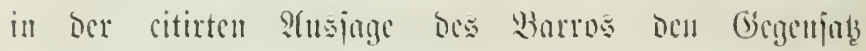

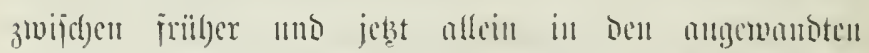

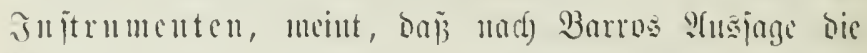
Junto offenbar bie $23 e i j e$ ju benbachten gejunden babe,

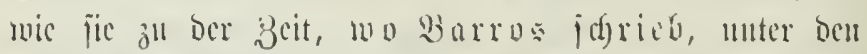

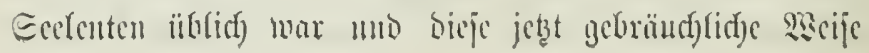




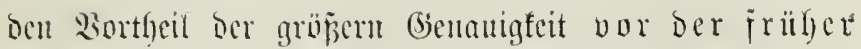

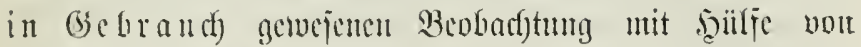

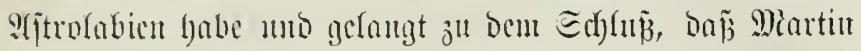

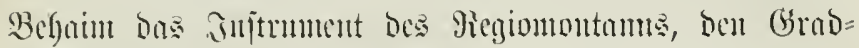

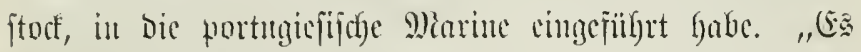

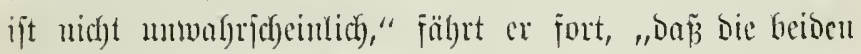

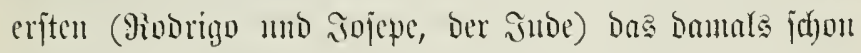

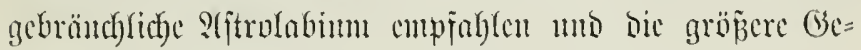

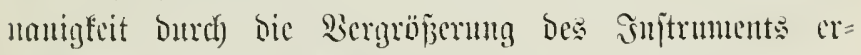

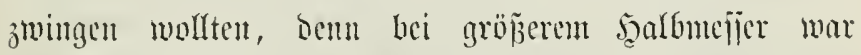
alferbings cine genanere s(blejunt möglich. Bisher waren

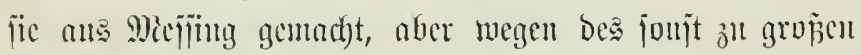
Metallgewichts mur in fleinerem Mapjitabe ansgejiil)rt.

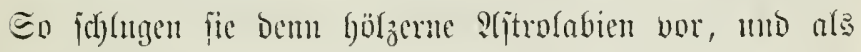
Saseo de Ganta feine Bieife antrat, nafmer, anjer ben gemöfyulidyen fleineren mejfingenen S(jtrolabien, and) cin gröperce fjölzernes an Borb. S(ber anj Ece liej jict)

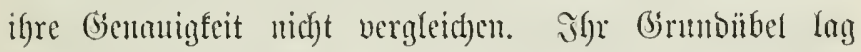

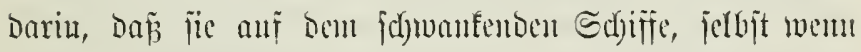

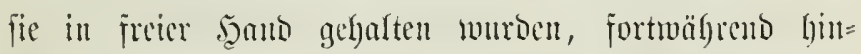

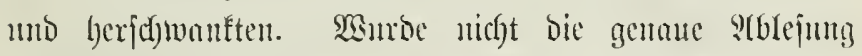

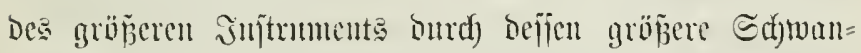
fungeu wieber angelyoben? Llu bice ju unterjuchen,

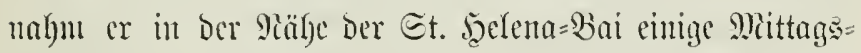
böhen auf Eee mo fticy dam an bas Sans, wo ber

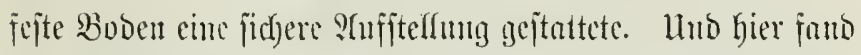
or, was er nicht ander: finden foumte, baj bie Beobad)tunģen, 


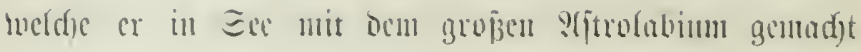

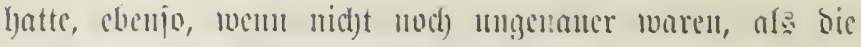

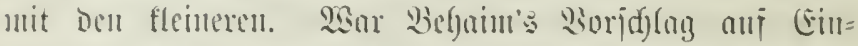

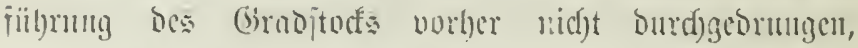

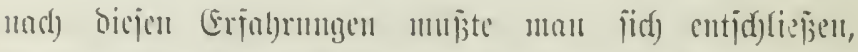

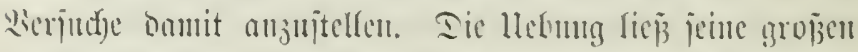

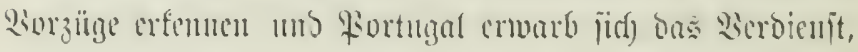

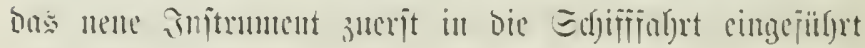
ill lyaben."

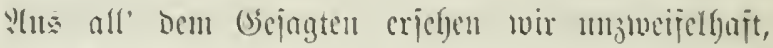

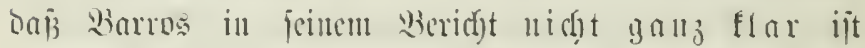

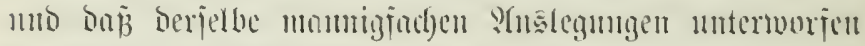

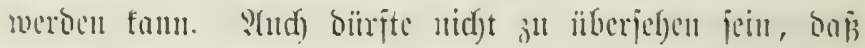

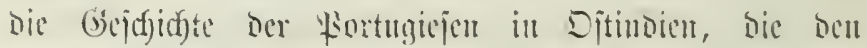

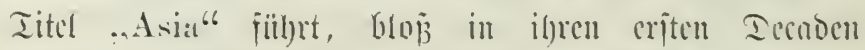

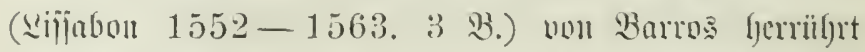

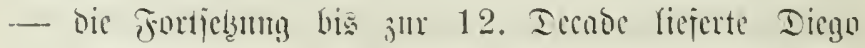

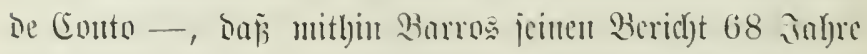

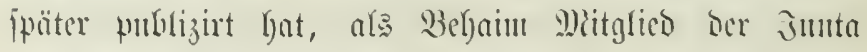

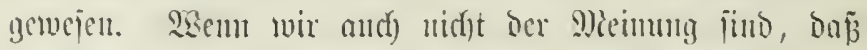

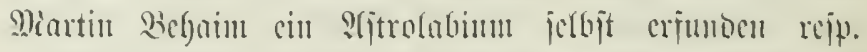
angefertigt, weil er mod) ju jumg gewuejen, noce cin grojes

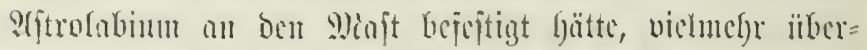

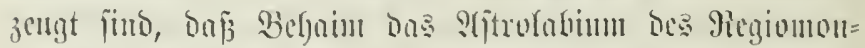

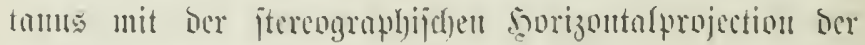

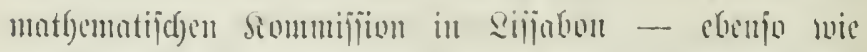
ben Jafohegtah bes gicgionmutums - vorgezeigt hat, 


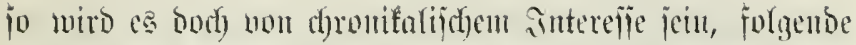

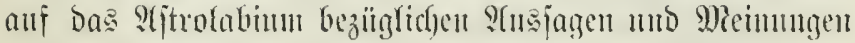
Gefonuter Eibriftiteffer bier anzufiulgren.

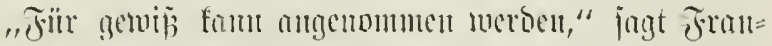
cišco be Borja Garcão=Stocfler in Fortugal ither Martin

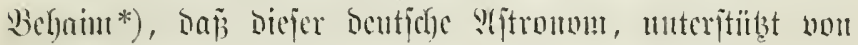

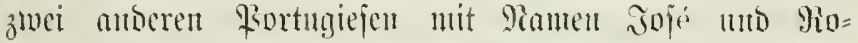
Drigo... Der Erfinder bes aftrolabiums war, weldes

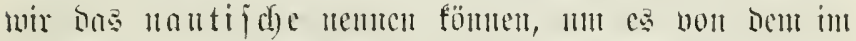

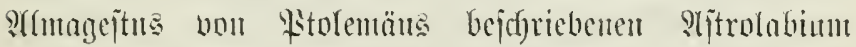

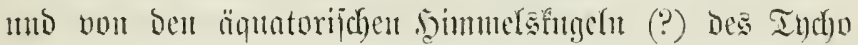

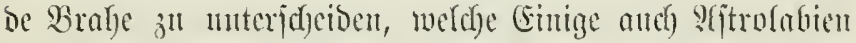
nemen, suftrumente, beren fümreidye Eimridftung mafyr-

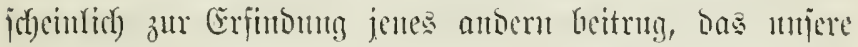

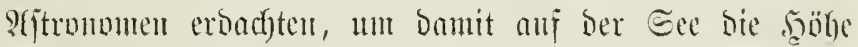

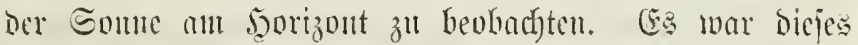
S(jtrolabium 1 mI fo nöthiger fïr bie Fortf̣chritte in ber

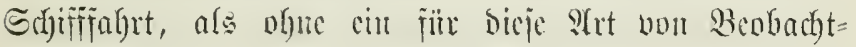
mugen geeignetes sujtrument jefbit bie fiemutuiz, baj

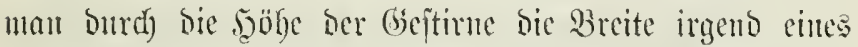
Drtes genan bejtumnen fömte, fïr bie Erbififagrt unfruddetbar getwejen wäre."

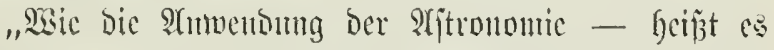

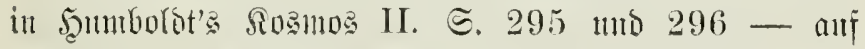

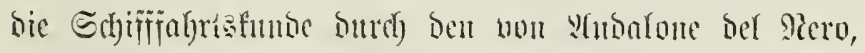

*) Eusaio historico sobre a originem e progressos das mathernaticas em Portugal, Paris 1819. 


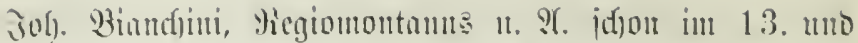

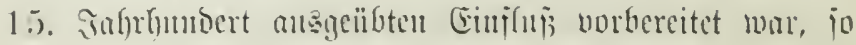

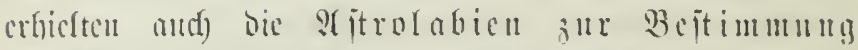

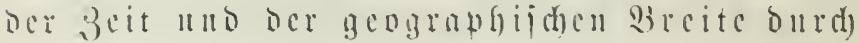
Meribiangübe, nuwendbar mi cincut iumer

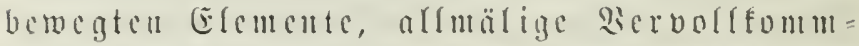

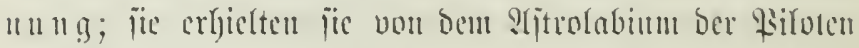

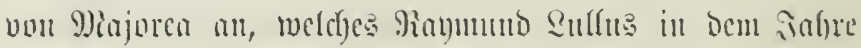
1295 in jeincr Arte de naregar bejoureibt, bis ju

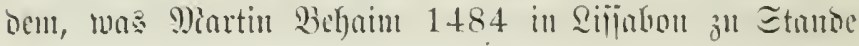

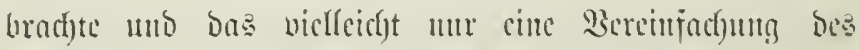

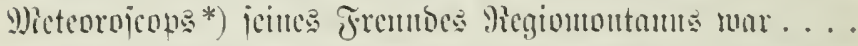
Ier Mame bes Astrolabion, wedeses Martil Bethaim

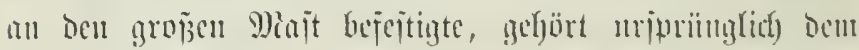
Sithuard)."

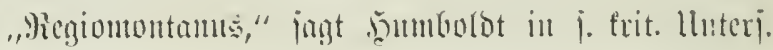
I. 224, 221; II. 295, "war Bamals beriifme ourdh

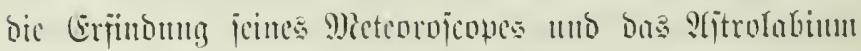

*) Das Dietcorojcop, obgleid) ber frorijont nud) in 3 in (sirnse eingetheitt, ift jeinem Buerfe nad) mejentlid) vom 2rftrolabium vers

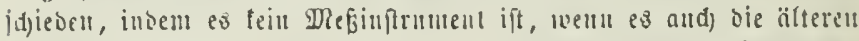
Ed)riftifter mit sem 2lptrolabium verued)elt ju babell fdeiuen. Tu Die Etelle ses veridollenen Megiomomtan'jdjen 21ftrolabimms, we?des

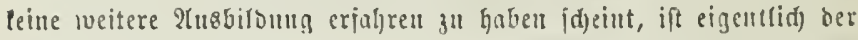

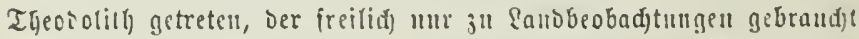
werben founte. Ier Certant ift ilbrigens and) bell Iffrofabium vermand, weil er bell Wsintel und) (5raben gient, aber ons Mejent.

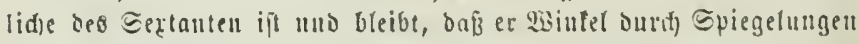

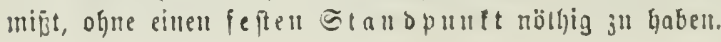




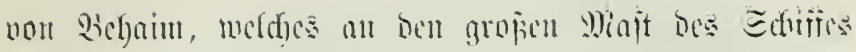

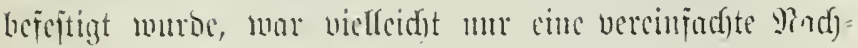

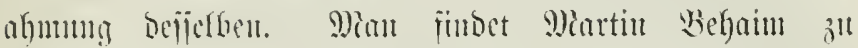

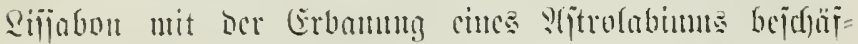

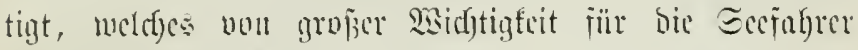

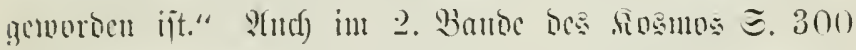

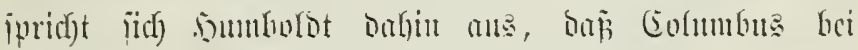

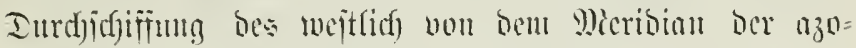

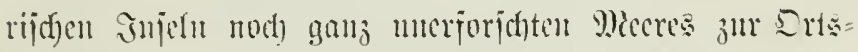

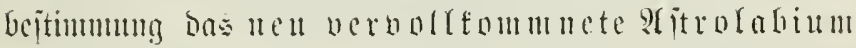
angemanst hat.

"Ias SGtrolabintm," joģt mijer berüfmeter Geograph

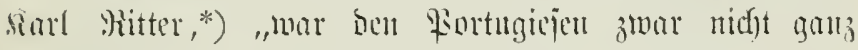

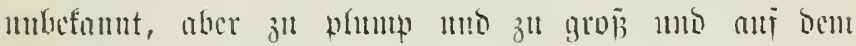

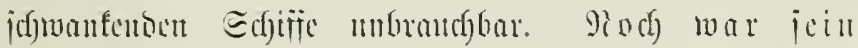
(bebraud bisher an bas anub bejdränft ge= bricben. Mentin Befaim, ber in den medfanijdyen Esereftätten der Pülnhlerger, bic bamals bic bejten

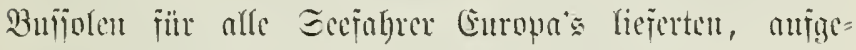

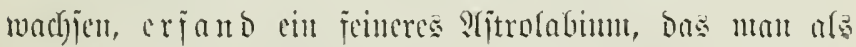

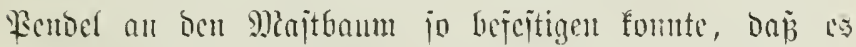

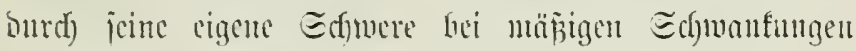

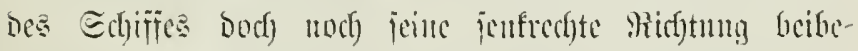

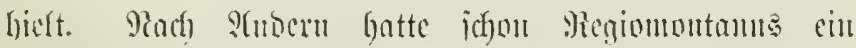

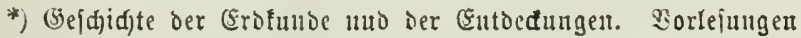

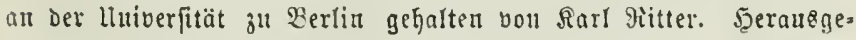
geben voul 5. थ. Daniet. Ferr. (5. Reimer. 1851. S. 254 u. 255. 


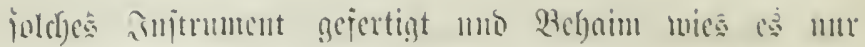

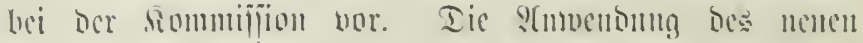

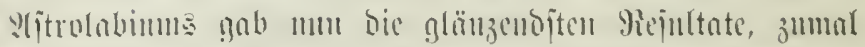

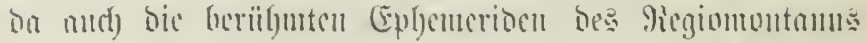

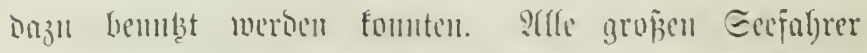

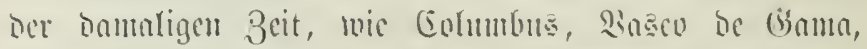

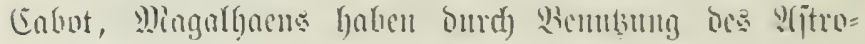

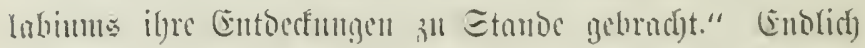

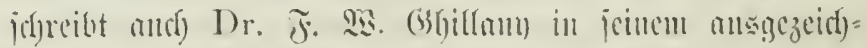

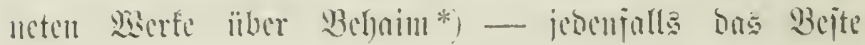

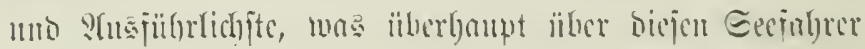
publicirt morben ijt - Folgentes: "Ritolcuntus ipridgt

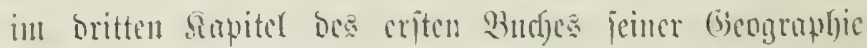

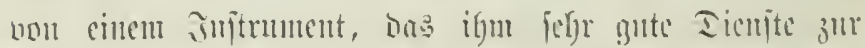

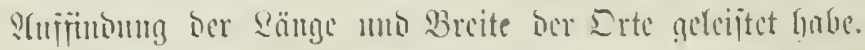

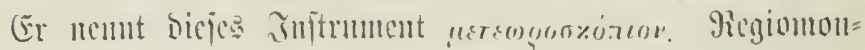

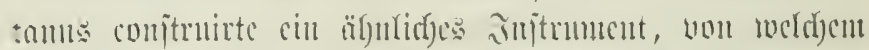

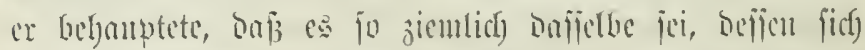

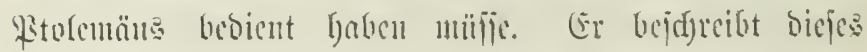

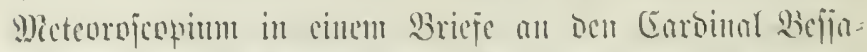

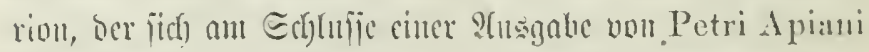
introductio geographica in Vemeri annotationes

7) (5ejdidyte des Eecfabrets Tritter Dartin Bebaim Mad) Dell ältcfeu Mrtunden bearbeitet uon I)r. $\%$. 23. (5)illany. Eiugelcitet

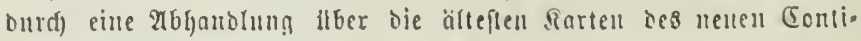

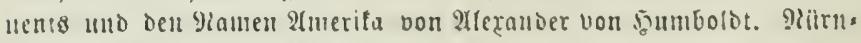
berg 1853 . ङ. 39 แ. 40 . 


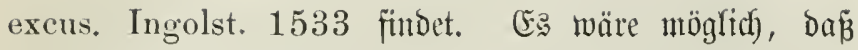
Bebaim biejes snftrument bei der Sommiffion in $\mathfrak{B o r}=$

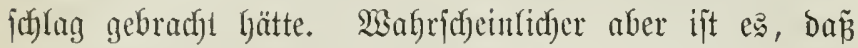
er Derjelben ein verbeffertes ats 9 Jetall gejertigtes, zum Intfhängen eingerid)tetes S(jtrolabium empjohlen habe,

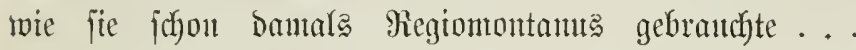
Dhne Bweifel ijt as biejes Jnitrument gewejen, weldjes

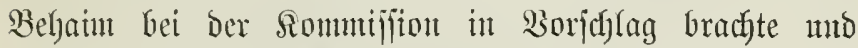

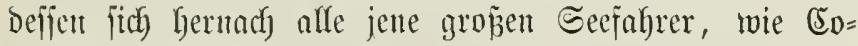
Yuthts, Basco de Giama, Eabot, Miagalfaens zc., Gebienten."

Bei ber SAntalyme vou Şumbolot, Ritter, Ģbillanty

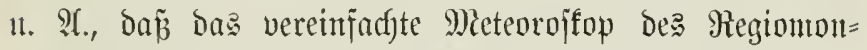
tants ober cin verbeffertes Sffrolabium won Martin $\mathfrak{B} e=$

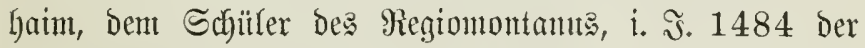
Sommififion ober Junta de mathematicos in Riffabon vorgelegt worben, faum baffelbe vor bem genannten Jafre 1484 fein verbreitetes gewejen jein und ijt wohl zuerjt

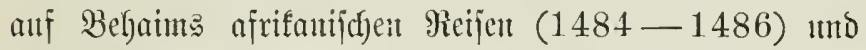
Daun fpäter auf Den Reifen Des SUterigo Besputcci (1497) und $\mathfrak{B a s c o}$ de Gama (8. Juli 1497 bis 29. Augujt 1499) in (bebraud getwejen. Da Martin Befarm bie

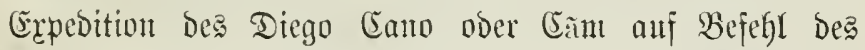

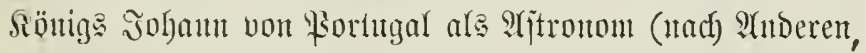
3. 23. nach Şartmann Sdjedel, als \$atron ober Bejeblä= Gaber eines Sdjiffes, ¡. Budf ber (Shronifent) auf jeiner Entbecfututgereije längs ber Wejtfiljte MCFrifas begleitet

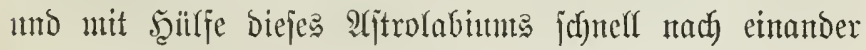




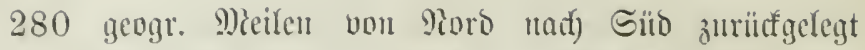

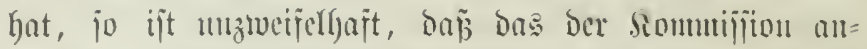
geblid) vorgelegte verbejierte 9(jtrolabium anj bicjer Meije

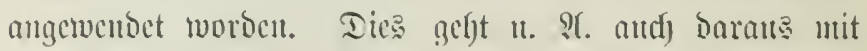
Sejtimuntheit Gervor, baj, wie ber berillyute italienifdje scput Maffec bericfetet*), bicjent Mietcorojep oocr ver=

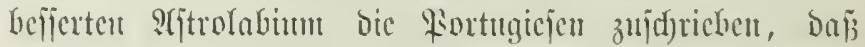
Jacol Enuts (Diego Enno?) glüdflidf) ïber bic Sinte

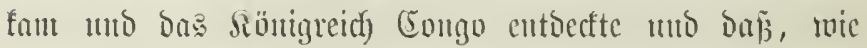
Trignzo**) Geridftet, Miartin Bebatm in Diego Canto's Begleitung von jecter afrifantiicten Sicije, bie fïf) bis iiber Das Cap Frio bintuts zum 22. Grand jüblityer

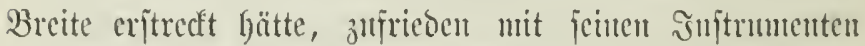

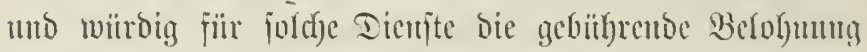

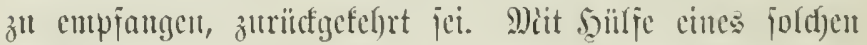
Jnjtruntentes war is aud), wic Shuretti jagt, ocm

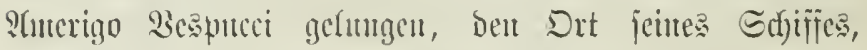
bas burd) eineu Stum verjof)(agen worben war, mitten

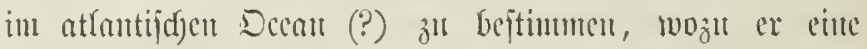
Bedectug des Mars nom Meonde beutube, dic

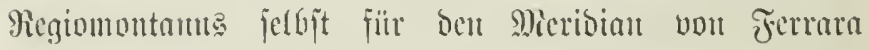
berecfunct hatte (,valendosi d'una congiunzione della

*) Hist. rer, indic. I. p. 11. Rergl, aud): Le istorie dell' indie oriental del P. Gio. Petro Maffei I, 17, 19. 20.

**) Sebastĩio Franciseo de Mende Trigozo, 21us den Medorias de litteratura Portugueza publicadas pela Academia real mas sciencas de Lisboa, tom VIII. Lisboa. 


\section{$-83$}

Luna con Marse, calcolata giì dal Regiomontano medesimo pel meridiano di Ferrara, seppe, dissi, argomentare in qual luogo la nave trovarasi*).

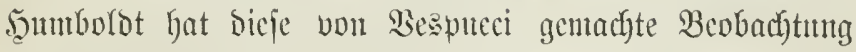
cincr Conjunction des Mars vom 23. Shtgujt 1499 (es ijt nicht gejagt, anj weldyem gimft ber Siüjte) nad)

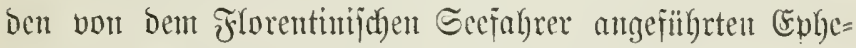

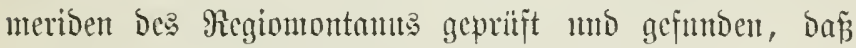
nicht Der geringite 3weifel barïlber obwalten fant, ob

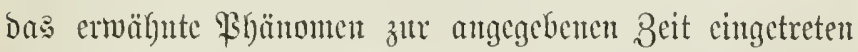
jei ober uticht. Dic Ephemerioen bes Regiomontants fïr bie Эaryre 1484-1505 jeben bieje Eonjuntion gerabe ani Mitternacht. Sie finto fïr ben Mieribian von

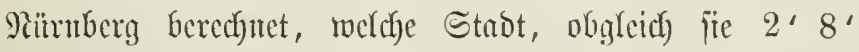
(3eit) öjtlich) bou Ferrara liegt, eben fo wic Mailant, Erfurt mo Branujurbeig damars als unter bemjelben Meribian gelegen betrad)tet mutroc. ${ }^{* *}$ )

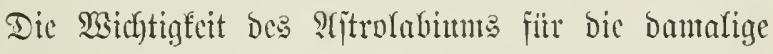
Beit geht itbrigens and jechlagento ats dem Briefe $\mathfrak{B e s}=$ putcei's an Mebici über bie oritte Picije hervor, inom er 1t. 2. jagt: "Win wären ohne Biel unngergeint (vagaboudi), went idf nicht mit $\mathfrak{b}$ ülfe bes âtrola = bium mo des Dutabranten (quadrante astrologica)

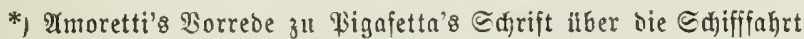
5. 266, f. Primo Viagin fatta del Cabaliere Antonio Pigafetta. Milano p. 208.

**) 5ุumboldt: Ririt. Itnterf. II. 505 แแ⿰ 512. 
Fïr meine uto meiner Gejäbrten Retmuty geforgt bätte." Эn einem anderen Brieje vom Briünen Sorgebirige den

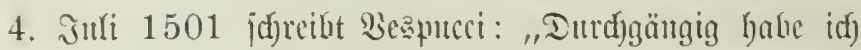

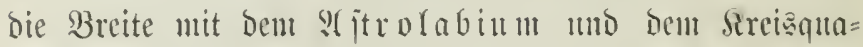
branten utad) eigenen Benbad)tungen bejtmunt." S(lud) it Der Berfaltumgananveijung nou 22. Mä̈rz 1508 wiro Beapucci beanftragt, bie Eteuermänner ,über den bie=

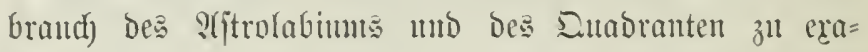

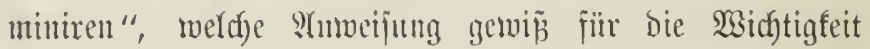

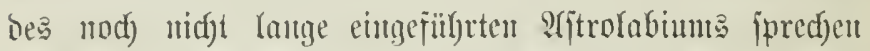
bürfte. Im 1lebrigen - ıno biejer Bemerfung it $\mathfrak{Z}_{e}=$

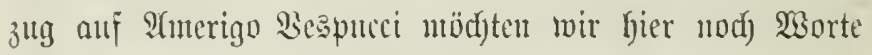
geben - ift es cine Sitge, wie jdon Sdjoner in Rürn=

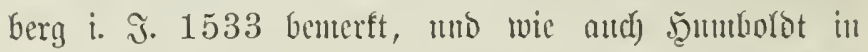

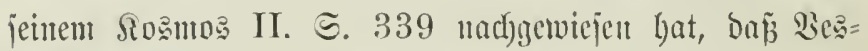
pucei bie Worte "Terral di Amerigo" lijtig in bie vout

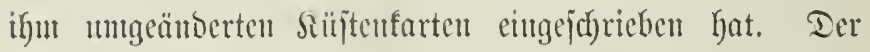
Rame "Anerici terrae" ift fifjon 1507 vou bent Geographen Warbjeentïlfer ober, wie er jid) ntad) ber damaligen (Selebrtemijtte gräcijirt naunte, Martimus IY Ylacomylus ans Freibury im Breisgan (Borfteber cuter Drufferei zall St. Die in Rotbringent) in einer fleitett

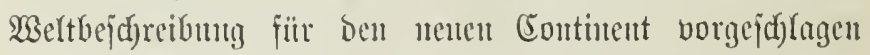
und Der Menen Welt fïr alle Beiten beigelegt worben.

Columbus judtete, bemerft v. J̧untbolbt, frit. lluteri. II. 6, indem er cin butd)aus unbefaunte sleer ourd)=

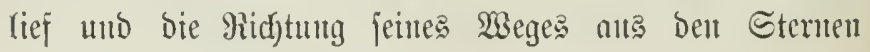


mit sülic des aftulabiums erforfate, das

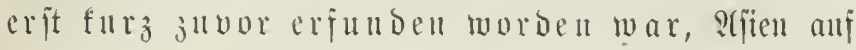

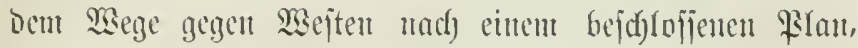
nid)t als :(bententer, weld)er fid) nad) (Sintoinufen ben Sufarl inberläpt. Columbus ijt befanntlid) autd) in bent iejten Gilanben gejtorben, die siijten von sfjien anfge=

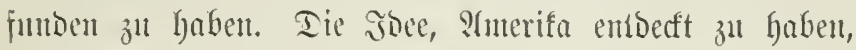
ijt ifut bis zu feinem Tobe frumb geblieben. Colunbus twax bergejtalt mit ber sobe exp̈illt (i. Sosmos II. 304),

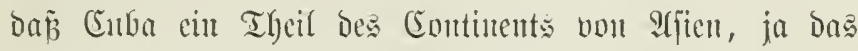
fiiblidbe Sathai (bie Provinz Manıo) jei, baj̃ er am

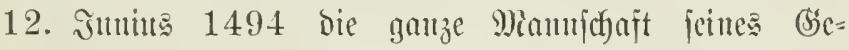
idrwabers fontwören fiej, "jie feten babon ïberzengt, man fömte von Cuba nad) Spanten zu Zanbe gehen" (venir de España por tierra); wer von denen, meldje es jebet bejantwören, einjt bas. (Siegentheil zn beganptent magte,

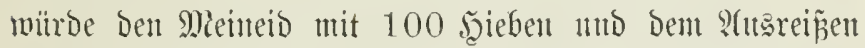
ber 3unge z"l bitînen lyaben." (Informacion del escribano publico Fermandez Perez de Luna in Navarrete: Viages y descrubimientos de los Españoles T. II. p. 143-149.) Я્a Columbus auf der eriten Expedition fidf ber sufel Enba näbert, glautbt er fidf

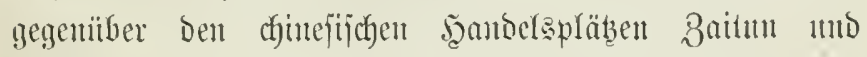

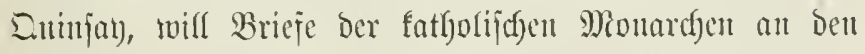
grop̈en Miongolen=CGyan (Gran Can) in Sathai abgeben

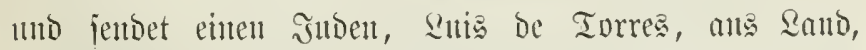

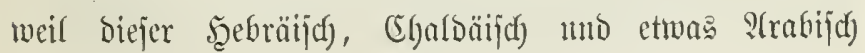




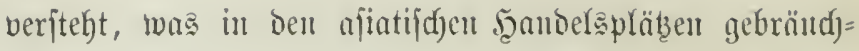

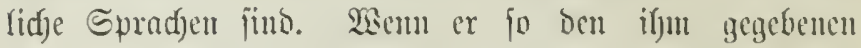
SHiftrag erfülfet, molle (rolumbur jogleid) stad) Spanten, jei es jur Eec iiber Cenlon mo rodeanto toda la tierra de los Negros noer zu \&anbe ïber Jerufalem

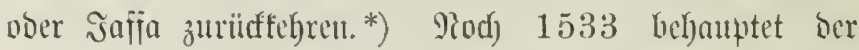

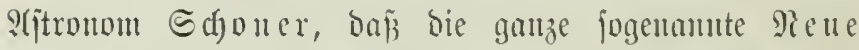
Welt ein Theil bou affien (superioris Indiae) jei und Dañ bie von Cortez eroberte Etnot Merifo (Temijtitan) nichte anderes jei als bie djinefifdye von Marco Polo

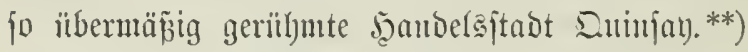

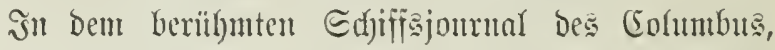
in dent oft des Etreites mit Stlonju Pinzon über Die

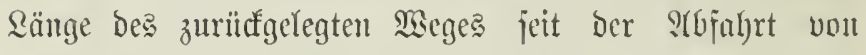
Ralos fomie ber gebrautden Sandulyrel (ampolletas) gebadjt miro, wiro weder bas $\mathfrak{L g}$, la corredera nod)

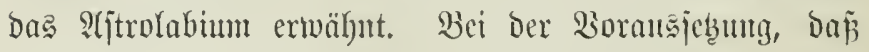

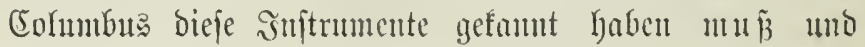

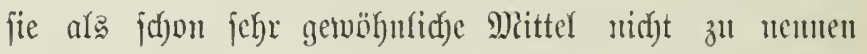
nöifig eradjtet lyat, wie z. 23. Mlarco Folo nidjt bes Thee's uno ber djuncifid)en Mianer erwälyut lyat, jo faum

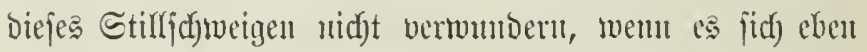

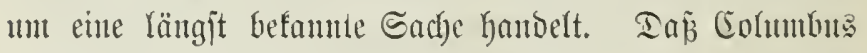

*) Reifeiournal des Eolumbus von 1492 in Navarrete Viajes I. 37,44 und 46 .

**) Ioannis Schoneri Carlostadii Opusculum geographicum. Norimb. 1533. Pars. II, cap. 1-20. 
auf jeiner gieije zur Drtabejtimutum bas nen vervoll= foumutete ?(jtrofabius antgewantot hat, Dafür ipridat

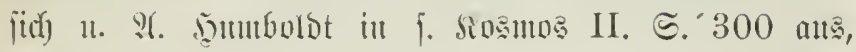
mie swir jafon bluen benerft.

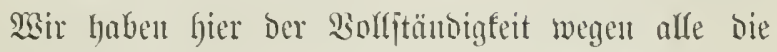
obigen SGngaben Der betr. Edyriftitefler in Bezun auf bas sfitrolahinm zujanmutengefteflt, ofue anf bie vielbetonte

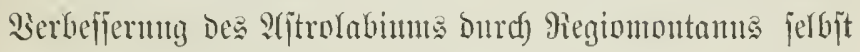

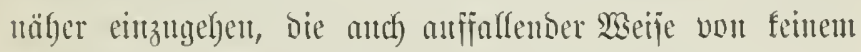
ber ohen gentanten Ed)riffifteller nähur angebentet wiro. Morin fat mun bicje antgeblidje Berbefjerung bejtanden? Un

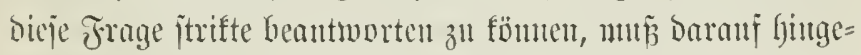
Deutet werben, Dañ bas : Sim bat. Ilnter s(jtrolabium verĩtano man, momit anch) bie mir getworbenen brieflicyent Mittheifungen

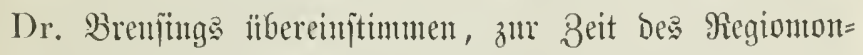
tauts bas, was man jeķt jtereographijáse Projection ber Sutgeloberfääcje in ber Ebene nemut. Dieje Projectinn

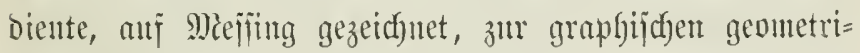

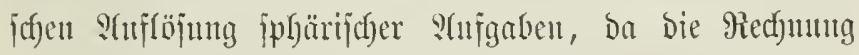
- Der reine Calcül - vor IHsbildung der Trigono=

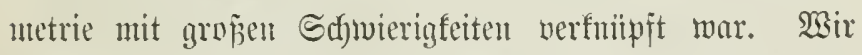
haben ïber bieje jtereographijdye \$rojection ein \$aerf von Dem berüfunten s(jtrontumen titnlemäns. Taffelbe be= ganbelt fie aber mu alz Polarprojection. Regio=

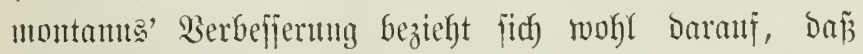
er ber (Exjte gemejen, Der fie and als bुorizontal= 


\section{$-88$}

projection entworien hat. Iieje Frojection hat Re=

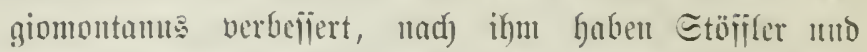
Bemuna Frijus (Astrolabium citholicum) bicjelbe be= id)riebert.

?tus bem ?(ltertfyume haben wir neben nuberen wertylojen Mettgoden bie Sutgeloberfläd)e in ber Ebene Darzutitellen oder ju projicieren (id) nente bas: Berebenen Der Sugeloherf(äd)e) bic jolgenben brei fjödjit widftigen ïberfommen, bie wir jest 1) bie Centralprojection, 2) bie orthograplifaje Projectiout, 3) bie ftereographijaje Projection menten. Dieje Samen haben jie jriiker nidjt

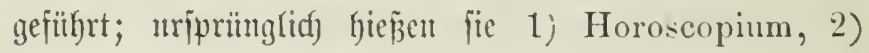
Analemma, 3) Planisphaerium. Leber bie betoen

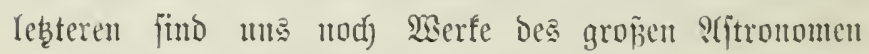
uno B'seographen Ptolemäus erhalten, leider nidyt in oer Ulipradje, jondorn in Iateinifdjer Lleberjeßzung, die erjt wicber ans bent S(rabija)en itberjebet ijt. Ier Pame "Ttereographijaje Frojection" riilfrt vom Sejutiten Fran= cois Aguillon her mto fintoet fid zuerjt in befien, ,Opticorum libri VI. Sntwerp. 1613. Erjunden ijt fie

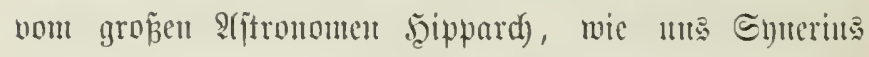
in jeiner Edfrift "De dono Astrolabii" berid)tet (Synerii opera ed. Petau. Lutetiae 1612. Fol. pag.

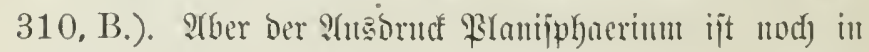

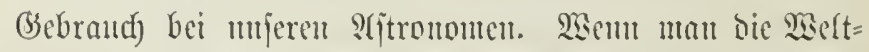
futgel in "Zwei Pfanijphyären" barjteflt, jo heipt bas in:

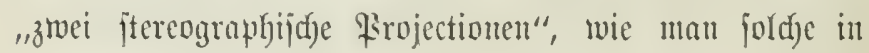


jedem 2ttras, z. B. Dem Etieler'fdyen, findet. Dieje ztwei

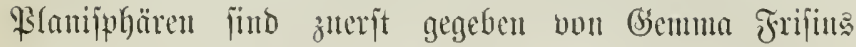
unb baum burd) Micrealor affgemein geworben. ${ }^{*}$ ) Weum ce aljo ım 1500 heifjt: Regiomontame habe das Siftrolabimu verfeffert, jo Geipt bas in mijerer jebigen Spradjmeife: Regiomontames lyat bic ftercograplji= ide frojection verbeffert. lints worin gat bieje

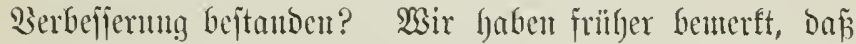

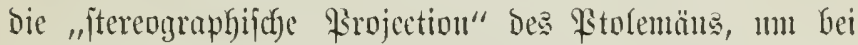
biejer Benemmung zn bleiben, lediglid) eine "polare" ijt. Man nuterjajeibet befanntfich bei alfen jenen Frojectionen 1) einte polare, wo bas 2 (nge im $\mathfrak{P}_{\text {ole }}$ ijt, 2) eine äqua= toriale, wo bns Stuge im $\mathfrak{Y}$ equtator ijt, 3) eitte fjorizon= tale ober zenithale, wie man jeb̧t lieber jag̣t, wo bas S(uge fid im Standpunfte des Beobadyters ani Der Erbe ober am f̧inmel befindet. Die Erofngel in "3wei Plani= iphären", movon idf oben fprad), ijt eine äquatoriale jtereographijoce Rrojection. Regiomontants gat, wie id) glaube, zuerjt bie horizontale jtereographifde

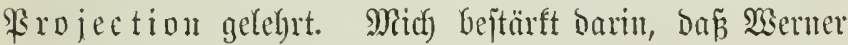

*) S. Joannes Stoflerinus Justingensis in: Elueidatio Fabrieae ususque Astrolabii. Oppenheim 1513. Fol. fạt Fol. XXX verso.

„Ptolemaeus appellat Astrolabium planam sphaeram aut planisphaerium ex eo, quod ut sphaera extensa in plano."

Jordanus de planisphaerii figuratione, Venetiis 1558 apud Aldum fagt im 2(njange: „Sphaeram in plano describere planisphaerium sive astrolabium nominamus. 


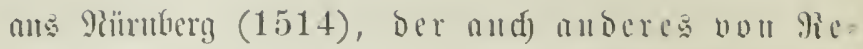

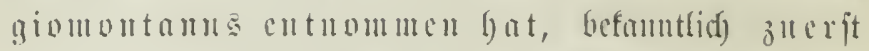
bic horizontale jtereographijdje Projection fïr Eandefurten

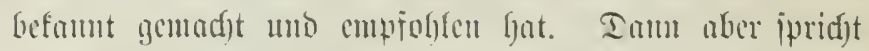

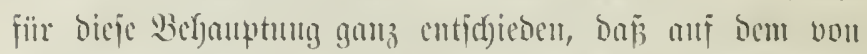

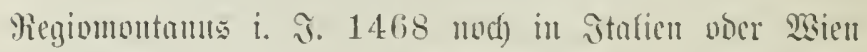

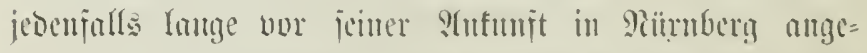
fertigten und stod) jekst anj ber Phïmberger Etmotbiblio=

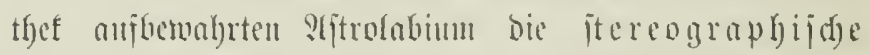
gerijoutarprojection angemanst ift, wie bies and

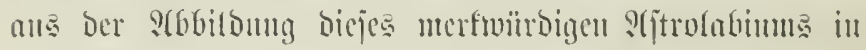

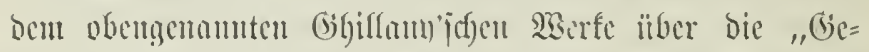
idjidfte obe Secfabrers Mitter Martin Bebaim" ganz

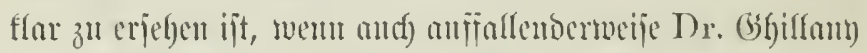
bicje widstige fecrographijd) nowizontalprojection des Reginntoutame nid)t crwälfut un betont hat.

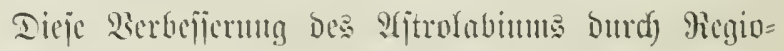
แontauns ijt fein geringes Sierbienjt, beun burd) bicje

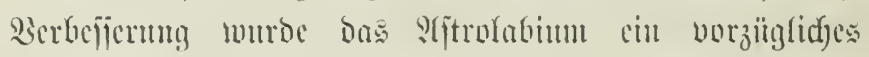

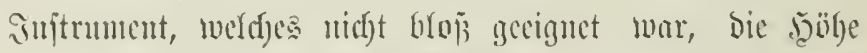

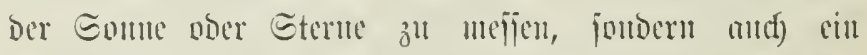
Mrittel bot, bieje Beobad)tungen jogleid) jut bemtsem,

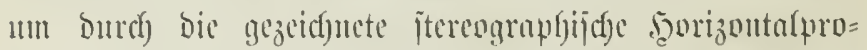

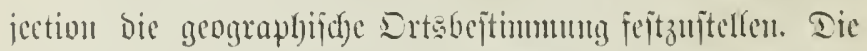

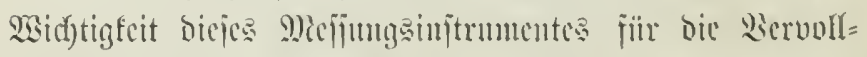

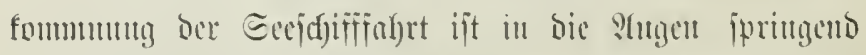
und brandyt nidjt näber bejprodjen z" werben. Sic 
ftereographijaje Projection biente frilger und bient and

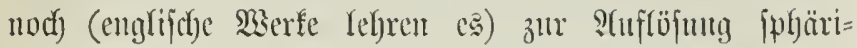

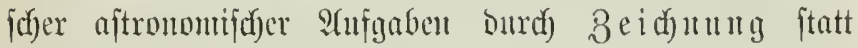
Durdf trigonontrifde Redunng. Die Rogarith=

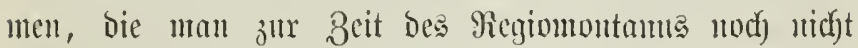

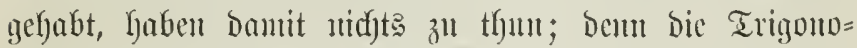
metrie war volfitäntoig ansgebildot, ehe bie Sogaritgmen erfunden murben; bieje twaren wieber mur cine Erfeid)= terung Der trigoudmetrifden $\Re$ edunngen. Die von Regiontontamis gezeiduete ftercographifade seorizoutal= projection biente bazu, die Frage ans Der ipljärifacen

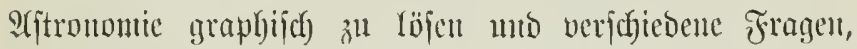

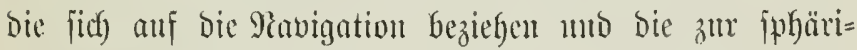
fäen arftronomie gefören, wurben immer graphifd) gelöft. In biejer jtereograplyijayen ,̧orizontalprojection lyat

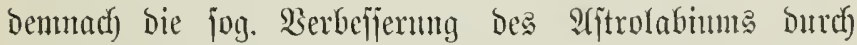
Piegiontontames beftanden, nicjt aber, wie man anjunefy= men geneigt ift, in einer Serbefiernug Des Sonfrnmentes

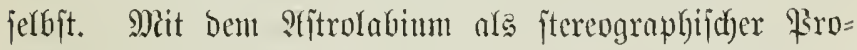
jection war eill affribabe verbunben, mit Dioptern ver= fehen, un Winfel zu mefjen; nebentbei war aljo baffefte aud ein Winfelmépinftrument. S(ther an biejer einf̣ad)en Borrid)tung ifit nie ctwas zu verbefiern ge= wejen uns baran fat and Regiomontants nidfts ver= befijert.

2łus all' Dem Bejagten geft hervor, Dap̃ Regiomonta=

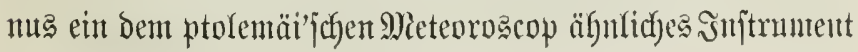


verfertigt hat (f. Brief bes Regiontontaum an Den Car=

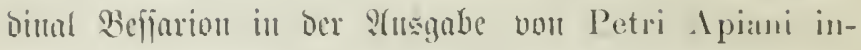
troductio geographicil in Verneri anuotationes

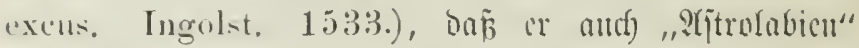

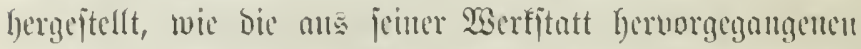
uno nod) jebet nuf ber Sü̈rnberger Etadtbibfiotget mif= bewabrten Inijtrumente bicjer stet bemeijen, boj or auj

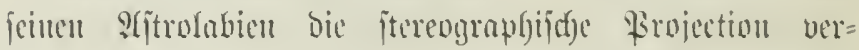

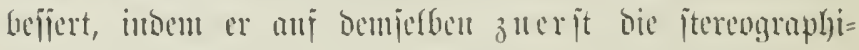

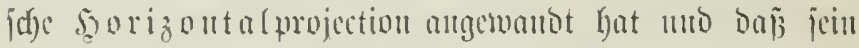

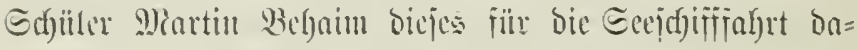
mals midftige Suftrument ber matgematijegen Smuta in Diffabon eben jo gut vorgezeigt unt cmpjoglen faben

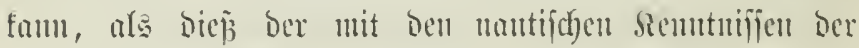
Entefaner mb Miajorcaner nertrante Robrigo mo ber

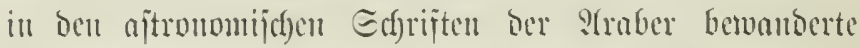
Sube sojepe mit bem domals gebräudf)(id)en s(jtrolobimm (ofne iterengraplijaje sonizontalprojection) getfan.

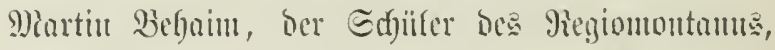
bat aber midyt mu bas Regibutoutan'jeges ?(jtrolabium mit ber verbefierten ftereographijchen firojection ber ma=

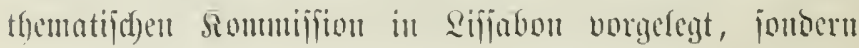
and) ben non Regiomontames erfundenen Safobsitab

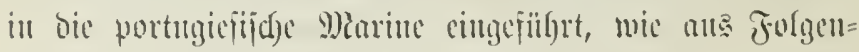

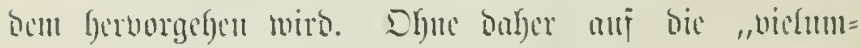

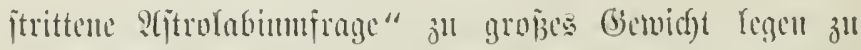

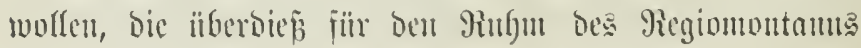


vou feiner entficheibentoer Bebentung ift, wiro ca vou

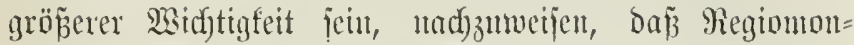

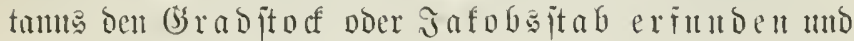
Befaim, jein Sdyuler, biejes Jujtument bes Regiomoutan in bie portugiejija Marine eingefübrt fat. Dr. Breming, Director Der Stener=

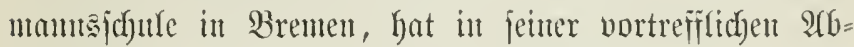

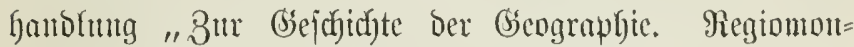
tants, Martin Berjaint mo ber Jafobsitaf.*) Diejen be= weis jtreng wifjenjdaftlid) ju füfyrelt verjudft und ijt Dabei vou folgenden Boranşeţutgen atsgegangen.

2Tufnüpfento an eine Steffe in ber Asia des. João Barros Dec. I. Lib. IV. b, bie Darauị hunbentet, da ja

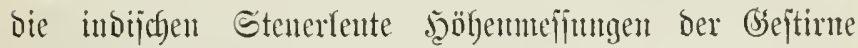
gefaunt, ans Der aber nidyt, wie cinige mambafte Greo=

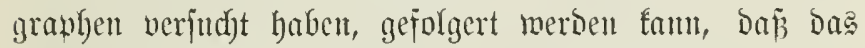

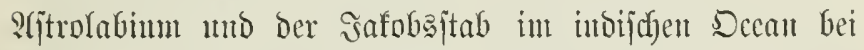
Anfunfit Der Rortugicjen bereite Gefanut getwejest voer baf

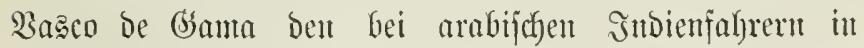
(bebrauch getwejenen safobajtab 1499 nad) Ermopa ge=

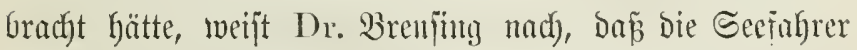
des Rothen Meeres unto des Indifden Deents weder

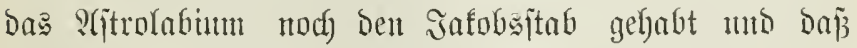
Basco de Gsama 1tm jo wentiger ntibthig hatte, dell Grad= jtocf nad) Ertropa zu bringen, als biejer bort fadjon feit

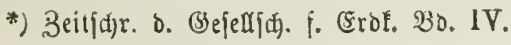




\section{$-94$}

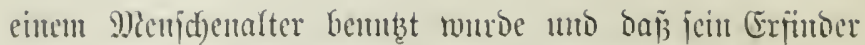
fein geringerer als giegionnontants gemejen. Dic genane

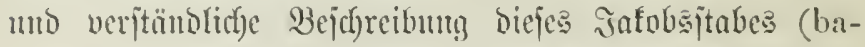

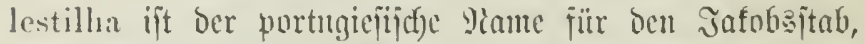

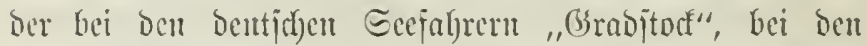
engliffchen cross-staft, bei beu iranzüjijcyen arbalite ficij) gibt Regiomntame in ber von ifm, wic man glanbt, bei befegentyeit bes in aabre 1472 erjojienenen

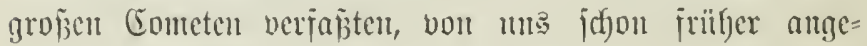

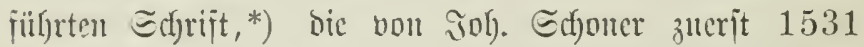
affein แnD ban ipäter mit mefren anderen Edyriften Des Reginmontonms vereinigt nod) eimmal im Jabre 1544 berausgegeben wurbe.

Regionoutaums, ber zuterĩt bic Conteten in ben

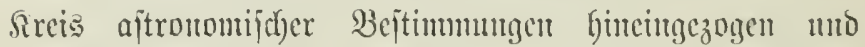
bei jeiner Stmmejentheit in Päirnberg $(1471-1475)$ Be=

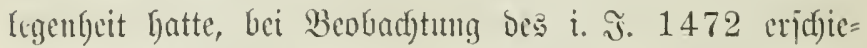
menen Conteten Iluterfud)ungen ïber bie Paralfarc biejer

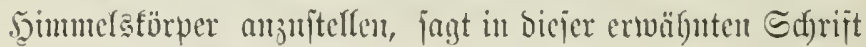
im Hrobl. XII.:

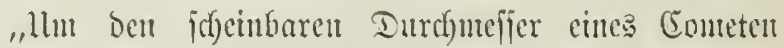
3u bejtimmen, mebure man einen glatten Etab $\Lambda \mathrm{B}$ mo theife ifn vou $A$ ans in glecidje Theile, je meljr bejto bejier. Bejejtige an ifm unter redftem $\mathfrak{B i n t e l}$ veridficb=

*) Joannis de Monteregio: De cometae magnitudine longitudineque ac de loco ejus vero probleinata XVI. 
bar eitren Duteritab CD, beflen beide SYme gleidy lany jein miff̈en. Theife ifn gentan in eben jofdye Theile, wie jie anj bem Stabe AB cingejchntitten jund; herejtige in ben Runften A mo C mb D Drei Bifiternadeft mo Das smitrument ift jertig. Die beofordytung aber ge=

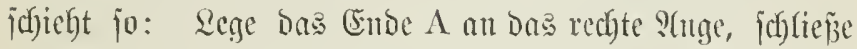

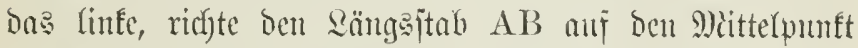
Des Cometen unto verjajiebe Detl Duteriftab, bis er Den Durchmefier bes Cometen gerabe becft. Daranf lies

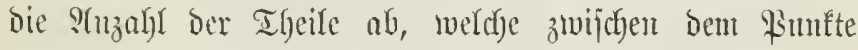
A mo dem Sueritabe CD liegen unb gehe bamit in einte eigens Dajür bepitumnte Iajel cin, Deren Beredfunng id) att einem anderen Drte erflären werbe, und Du findejt Den Durdfuncjier Des Concten."

"Dieje Bejureibung bes Grabjtofes, jagt Dr. Bren=

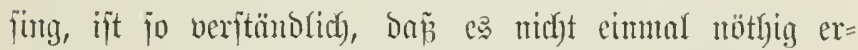

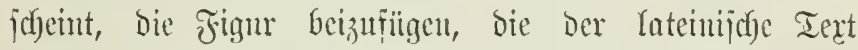

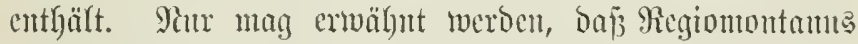
Den Duterjtab in 210 Theife theilt mo ban bie Theilung alf Dem Rängsitabe bis zu 1300 gebt. Wăa bie er=

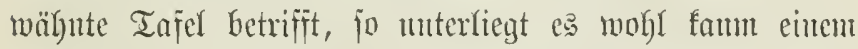
Zweifel, baj bamit bie Tajel ber trigonometrijachen $\mathfrak{T} n=$ genten gemteint ijt, auf Der ja bie Beredfuntug ber $\mathfrak{B i n f e l}$ bei biejen Injtrumente Geruht und bic unter bem $\mathfrak{R a}=$ men: "Tabula fecunda" vou Reginmontamts in bie Wiffenjaflaft eingefülfrt ift. WEenn aber bieje Schrift iiber

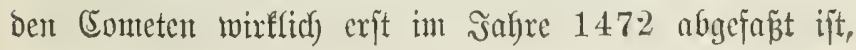




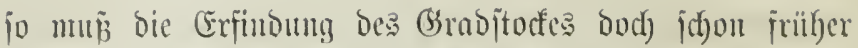
inflen. Dem in ben von Ectgoner im Jabre 1544 hermegegefrenen Beobad)tungen Riegiomontan's findet fid) unter bem Jahre 1471:

Die 9. Septemhris mane Mars ab humero dextro Orionis 210: 674; a capite Gemini praeced. und septentr. 210:622, wo bic 3ahf 210 bic

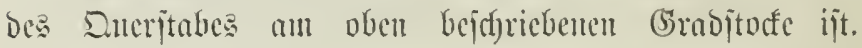

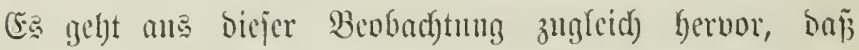

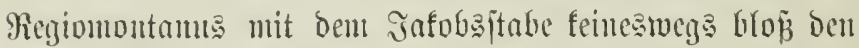
Durdfmefjer des Cometen gemejpen hat, fontorn daj or

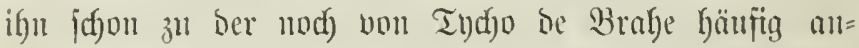
ycwenocten Drtabejtimmung eines (sejtimes burd) MRejiun jeines ?(bjtandes von zmei anberen Gemutze."

Regiomontame Gatte jelbit bem Jujtrument feinen

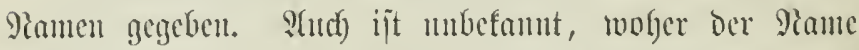
Jafobsitab genommen jein may. Diejes Injtrunent (Winfelmeffer) nemt Bernhard Malther rectungulum astronomicon, ber Pämberger Miathematifer Johanuts

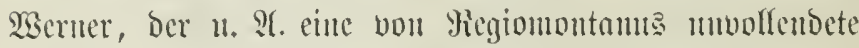
lleberjekzıng Der beographie bes Rtolemäus 1514 mit

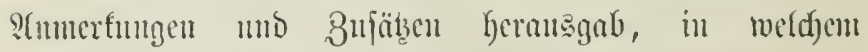

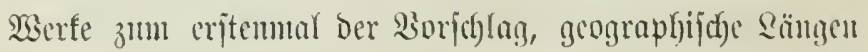

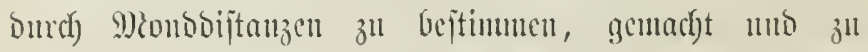

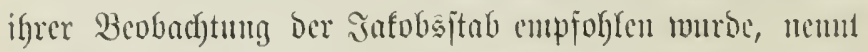
ea radius visorius ober observatorius mo STpian be= zeid)net is alg baculns astronomicus und radius 
astronomicus. Iluter Diejeu Yeţteren 9amen, Der unter

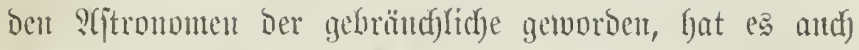

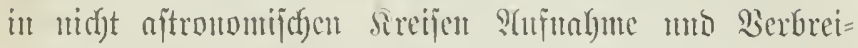

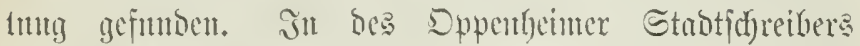
Infob Sibbd: "Geometrey, von Eïnftlidyen Mefient. Maninz 1535." findet Dr. Burnfing juerit dent Manten: Safobaftab. "Schwerlich wirs Sïbel's Bormame onzu Beranlafintug gegeben haben; follte vielleidht barin eme Quppielung anf Senefis 32, 10 riegrn, mf ben atranti= idjen Dcean als sorban, mb bie nene mo bie alte Welt als die beiben seere? Daj̄ bie Epanter diefen

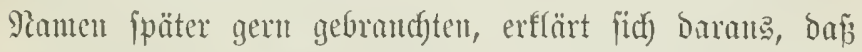
St. Sago ifre Nationalgeiliger ift." Doppelmanx, ber

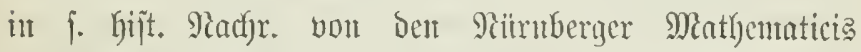
S. 7 aut anf bie, wie oben benterft, bou Regionton= tamts in 9ämberg zn Stmbe gebradyten snitumente: Radios Hipparchicos noer Ptolemaicos, Regulas Ptolemaei, Armillas, Quadrata, Geometrica, Torqueta

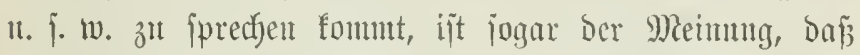
dic erfte (s)attung ajtronomijoser Jnitumente sine von den ältejten fei, Deren Erfindung verichtedente Prutoren dem Patriardyen Jacob (?) zu eignen, Dafer ntan es ins= gemein Baculum Jacobi nemt, weld)es man fonft, ba es

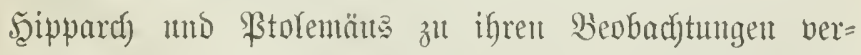
wenbet gaben follen, and Radium Hipparchium unt Ptolemaicum, Dann aber and Instrumentum Trianguli und Rectangulum neunt. 
"Obwobl won alfen erwägnten Edfriffiteflem, be= merft Dr. Breujing, 9iiemanto des Regiomontunts ale eigentlidjen Erfintoers gebenft, jo war derjelbe als joldfer bod) nidyt jo vollitändig vergejien, als bieźs hent= zutnge ber Fall ijt; und es ijt von lejonderer Beben= tung fïr uns, baj fid gerabe in Rortugal jein ?hoenfen erfalten hat. Roun jagt in feimen Berfe: De regulis et instrumentis (Conimbr. 1546.) Lib. II. Cap. 6, wo er ben Grabjtocf bejdreibt: Ejus fabricam atque usum tradidit Johannes de Monteregio in libro de Cometa. Dâj ber Grabjtof ridjt jojort alfgemein CEingang gefunton und z. B. in Spanien nody um bas Jahr 1550 unbefaunt mar, barf แns nidjt Wuntoer nebment, hat es dod) mefr als 50 safre be= burft, ehe ein im Berbältuif ungleich vollfommeneres sntitrument, als cas ber Grabjtocf im Bergleiche mit bem Pljtrolabium war, elje ber Scadren'idje Spiegeloctant ben Grrabitoff uno den Dabisquabranten verorängt hatte."

Rommen wir jeb̨t zum Sdjluzppunft unjerer Mlo=

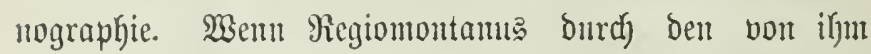
erfunberten Grabjtocf ober Jafobajtab, ber währent breier

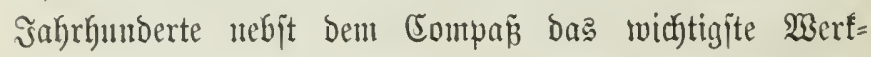
zenI in Den Seänden ber Seelente gemejen ift, der See=

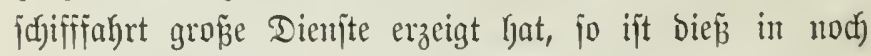
gröperem Grabe von den Eplemeriben Des Regin= montanus zu jagen, bie weber mit bem âjtrolabium mod) mit bem כafobsjtab etwas zu juaffen haben. 


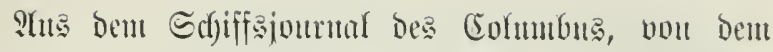

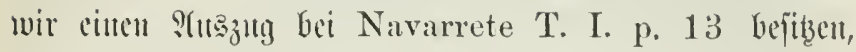
Defien Berichte erjt 1825 bis 1829 verifffeutlidyt wordeu,

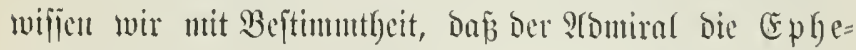
meriben des Regiomoutan au Bord gefabt;

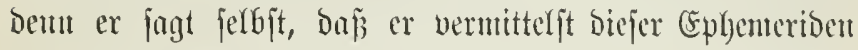
Den Eingeforenen eine Mondinfterni

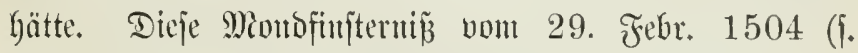
Bud der Profecias des Columbus $\mathfrak{B l}$. LXXVI, Ie $=$ itament des Diego Mendez, Navarrete T. I, p. 325, T. II. p. 272, Vita, cap. 103) Gatte Cofumbus orei Tage zunor bell Sttbinutern anj Santaica vorkergefugt,

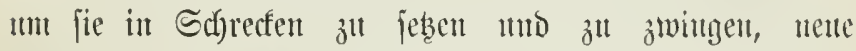

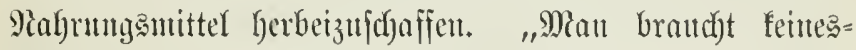

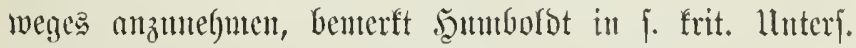

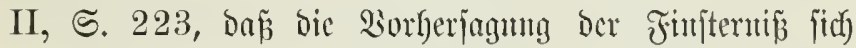
auf eine ferbftändige Berechnung des Coluntbus grïn= Dete; bent der Stomiral hatte nümlich ofue 3 weifer

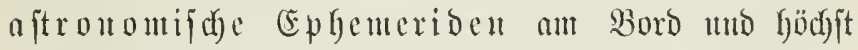

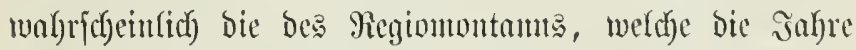
1475-1506 unifajien. Dieje stmafyme crbält um jo

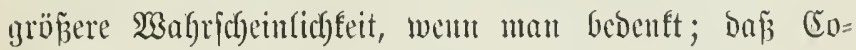

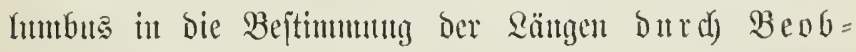

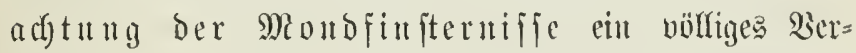
tranten febte und er jidf fidjon in bem Tagebuthe feitner

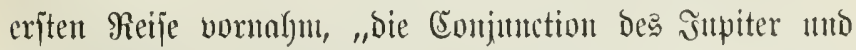
Mercur und bie Sppofition Des Snpiter zu Geobadfent", 


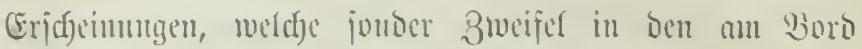
jeims Edjifies hefindiden Enhemeriben angegeben maren.

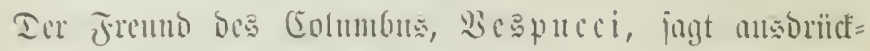
(id) in cutem Bricfe nu Lurenzo di Pierfrancesco de Medici (Bandini p. 72), baja or fidf mährento ber Jubre 1499 m 1500 , Des bour (iiovamni de Mon-

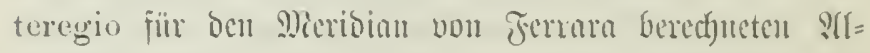
manadys" Gedient bat."

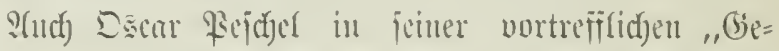
idfichte Des Zritulter Der Entbecfungen" jagt Є. 118,

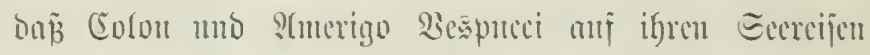

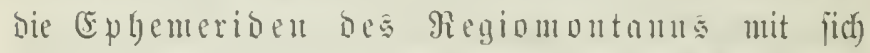

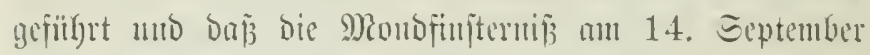
1494 ifmu (Solumbus) cinen wejtlidyen s(bjtano bur Jnjel Enona an Dor Sildojtipitze Şaiti’a vour Sap Ean Sirucente von $82^{01 / 2}$ (întt $59^{\circ} 40^{\prime}$ ) 1 mo bic Serfinjte= rang wout 29. Februar 1504, weldac Cormulus Den Eingeborellu Jamaifas a Expeneriben voransiagte, einen mejtlidfen s(bitanto

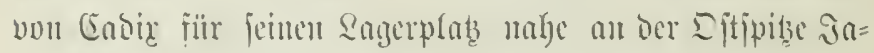
maica's von $108^{13} 4^{-3}$ gegeben bätte, wälgremo or mur $70^{\circ}$ finden burfte (Navarrete Coleccion, tom. II, 1, 272).

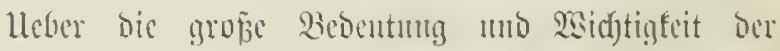

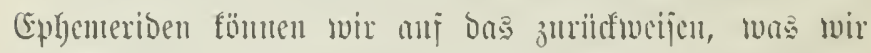

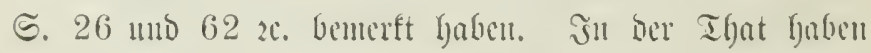
bie mit bent alfgemeinften Beifall aufgenoumenen (Ephe= meriben bes Regiontontam, dic Solnmbus offenbar bei 


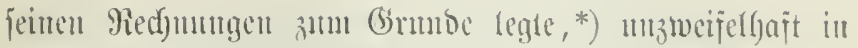

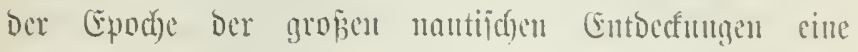

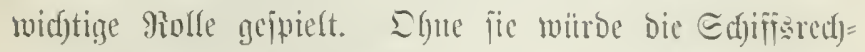

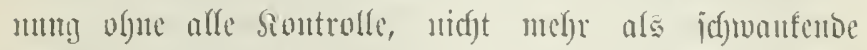

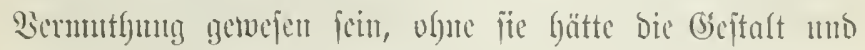
Sage ber nementoceften Rämber midyt jo Galo vergeidutet merben füntul.

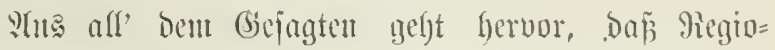
muntames nicjt mur afs ciner ber eifrigiten Befürberer

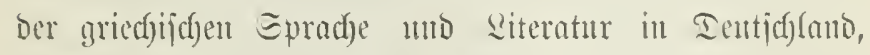
Fermer Der Mathemutif, Der STfgebra mo Irignnontetric,

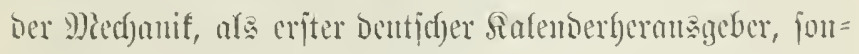

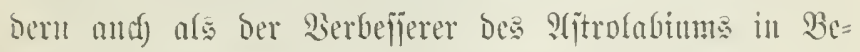

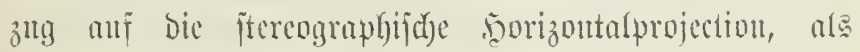

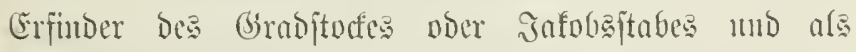
3erfafier ber meltberïfunten (Enhemeriben zil betrad)ten

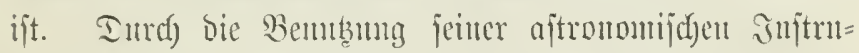
mente mid vornefunlict) feiner Ephemeriben hat Regio=

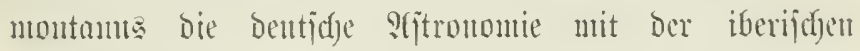

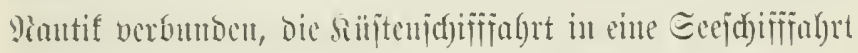

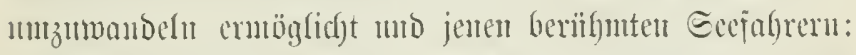

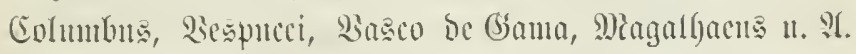

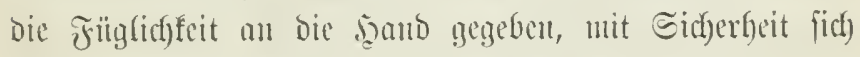

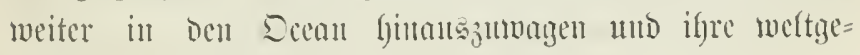

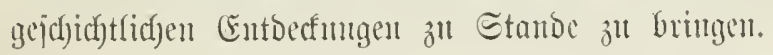

*) ๔. Şumborot, trit. Unterf. I. ๑. 541 


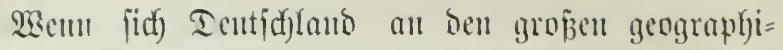

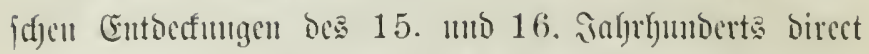
uicht betheifigt hat, jo finto es bod) bentidge (jelebrte

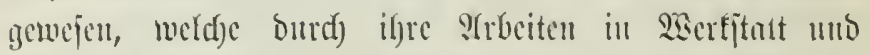

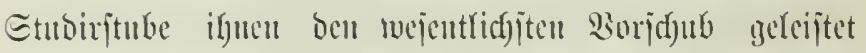

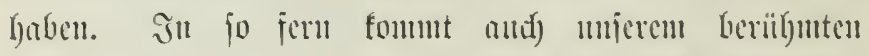

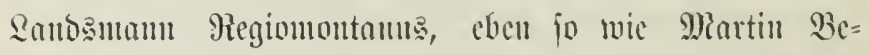
haim, fidferlid) bns Berbienjt zu, ein Borläufer bes

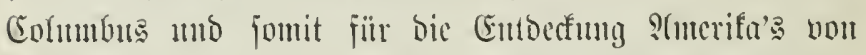
mejentfid)em sentzen gewucjen zu jein. Sn biejer $B \mathfrak{e}=$ ziebmug haben, wie id) f(d)on in einer frither erifjicnenen Edjrift*) bemerft, neben oen Stalienern, Epanicrn, Portn= giejen unb Franzojen and bie Deutiden in Folge ber

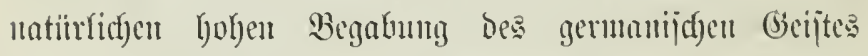
Theil an ber Ehre anf bie Entocefuny !nto Entwidfumy Shutcrifn's cben fo bebenteno als wohlthätig cingemirft zll habert.

Wie bie Solofiarbiijten des Regionnutants in ber

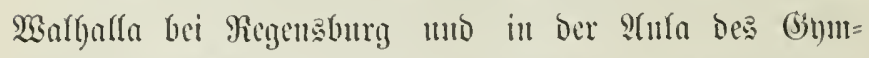

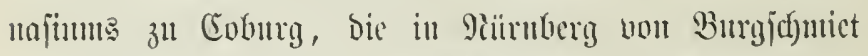

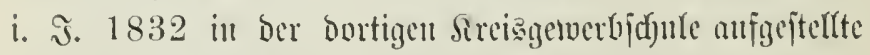

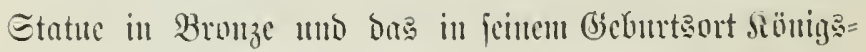
bery an 12. September 1871 entyillte Stantobild bie Serbienjte des beriifnten Mames verewigen mo bieje

*) Martin Bebaim alls Wiluberg. Der geiftige Entiecfer 2Imerila'8. Bon $\mathfrak{A}$ lę̧ander Biegler. Dresdell 1859. 


\section{- 103}

fichtbar vor miferen SThgen are eine Erinuerung an bie vergangente grof́ze 3eit Ginjtelfen follen, fo möge audf) biejen vorliegenden lluterju(f)utgen vergöınt jein, einen menten, frifd)en Lorbeerzweig zu ben briibentoen Siranze

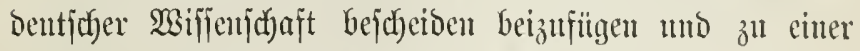

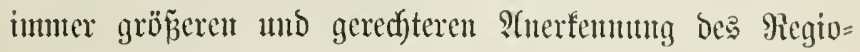

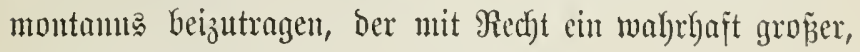
Dentjuer Maum genaunt werben faum. 
Dresden,

Frnte won 15. Syeintist 


Q

143

M9Z5

Ziegler, Alexander

Regiomontanus, Joh. Miller aus Königsberg in Franken

P\&A Sci.

\section{PLEASE DO NOT REMOVE} CARDS OR SLIPS FROM THIS POCKET

UNIVERSITY OF TORONTO LIBRARY 
(3) 3 s.

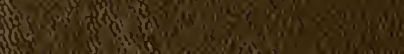

3)

Mon was

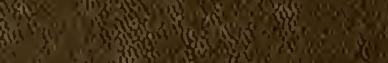

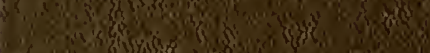

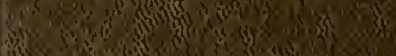

6)

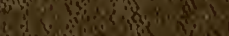

3 in

13 m.

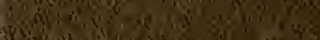

and an a

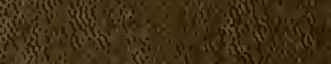

3. 3 ing

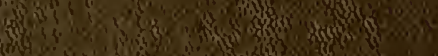

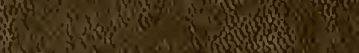

(1) 3 ,

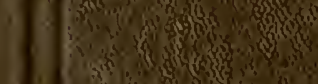

in 10 and

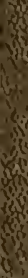

s.thing

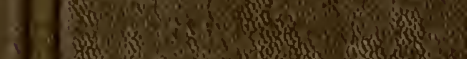

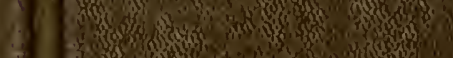

a.

isum

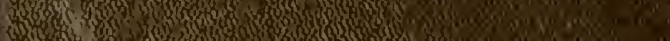

is 